Convección natural y transferencia de calor en medios libres y porosos

\title{
TESIS
}

que presenta la

M. en C. Elsa Báez Juárez

para obtener el grado de

Doctora en Ciencias (Matemáticas) 



\title{
ATI
}

UNIVERSIDAD AUTONOMA METROPOLITANA UNIDAD IZTAPALAPA

\section{Convección natural y transferencia de calor en medios libres y porosos}

\author{
Tesis que presenta la \\ M. en C. Elsa Báez Juárez \\ para obtener el grado de \\ Doctora en Ciencias (Matemáticas)
}

bajo la dirección del

Dr. Alfredo Nicolás Carrizosa

Departamento de matemáticas 

A Dios y a la vida.

A mi hijo Francisco.

A mi madre y hermanos.

A la memoria de mi padre

A todos los que me han apoyado de tantas maneras 


\section{Agradecimientos}

A través de estas líneas quiero agradecer profundamente al Dr. Alfredo Nicolás Carrizosa, director de esta tesis, por su guía, atención, apoyo, paciencia, respeto y entusiasmo a lo largo del desarrollo y elaboración del presente trabajo. Ello me permitió llevar a buen término esta tesis, el cual es sólo un pequeño escalón del interminable y estimulante camino a seguir en la investigación.

También deseo agradecer a los profesores: Dr. Roland Glowinski, Dr. Alberto Soria, Dr. Héctor Juárez y Dr. Eduardo Ramos por su tiempo, disposición, apoyo, sugerencias y comentarios hechos para mejorar la presente tesis.

A mi familia, amigos, profesores y compañeros les agradezco su confianza, estimulo, comprensión y cariño, que me fue manifestado con frecuencia y de diversas maneras.

Finalmente, agradezco al CONACYT por el apoyo que me otorgó para realizar los estudios de doctorado en la UAMI. 



\section{Contenido}

1 Introducción 3

2 Planteamiento del problema 13

2.1 Relaciones básicas de la mecánica de fluidos en medios libres y medios porosos . . . . . . . . . . . . . 13

2.2 Aproximación de Boussinesq . . . . . . . . . . . . 16

2.3 Problemas Adimensionales . . . . . . . . . . . . 18

2.3.1 Parámetros adimensionales y convección natural . . . 21

2.3.2 Transferencia de calor y Número de Nusselt . . . . . . 24

3 Esquemas numéricos en variables primitivas 26

3.1 Esquema numérico en medios libres . . . . . . . . . 27

3.2 Resultados numéricos . . . . . . . . . . . . . 32

3.2 .1 Caso Térmico . . . . . . . . . . . . . . 33

3.2 .2 Caso Isotérmico . . . . . . . . . . . . . . 43

3.3 Esquema numérico en medios porosos . . . . . . . . . 63

3.4 Resultados numéricos . . . . . . . . . . . . 65

4 Esquemas numéricos en variables secundarias 73

4.1 Ecuaciones adimensionales en variables secundarias ...................... 73

4.2 Esquema numérico en medios libres . . . . . . . . . . 77

4.2.1 Resultados numéricos . . . . . . . . . . . 80

4.3 Esquema numérico en medios porosos . . . . . . . . . . . 91

4.3.1 Resultados numéricos . . . . . . . . . . . . . 92

5 Conclusiones 114 
Bibliografía

115 


\section{Capítulo 1}

\section{Introducción}

Este trabajo trata sobre la solución numérica de flujos térmicos de fluidos incompresibles y viscosos, fundamentalmente los relacionados con convección natural, tanto en medios libres como en medios porosos, gobernados matemáticamente por la aproximación de Boussinesq no-estacionaria. En medios libres los métodos numéricos empleados pueden restringirse al correspondiente caso isotérmico, los cuales son gobernados por las ecuaciones de Navier-Stokes.

La convección es un fenómeno que involucra el transporte de masa y de energía térmica en un fluido, y se presenta con mucha frecuencia en diversas áreas del conocimiento, incluso en nuestra vida cotidiana, en una gran variedad de escalas, White [1], Phillips [2] y Lin y Segel [3]: en pequeñas escalas puede observarse en una olla con agua hirviendo o en el aire caliente que se levanta del pavimento y que provoca imágenes distorsionadas; mientras que en escalas mayores se presenta en la aparición del viento, en la circulación global de la atmósfera y de las corrientes marinas, en el movimiento del manto terrestre, así como en la migración y acumulación de hidrocarburos en yacimientos.

En los ejemplos anteriores, el fenómeno de convección tiene como punto coincidente y característica clave la diferencia de temperaturas dentro de un fluido o mezcla de fluidos (gases y/o líquidos), que se localizan en una región bajo la influencia del campo gravitacional, lo cual generará diferencias de densidad, haciendo que el fluido menos caliente y más pesado tienda a hundirse, debido a la fuerza de gravedad, y éste empuje, a su vez, al fluido más caliente y menos pesado hacia arriba. Este tipo de fenómeno se conoce con el nombre de convección natural o libre, Landau y Lifschitz [4] y Bear 
[5]. Si la cavidad o región que contiene al fluido está formada por un medio poroso (el cual se caracteriza por ser un material sólido con gran cantidad de pequeños poros, algunos de los cuales están interconectados), se habla de un fluido en un medio poroso; en caso contrario, hablamos de un fluido en un medio libre. En el primer caso, el movimiento del fluido se verá restringido al espacio disponible dentro de los poros. Ejemplos de fluidos en medios porosos son los hidrocarburos y el agua de mantos acuíferos moviéndose a través del subsuelo, y el aire que circula entre los granos almacenados en silos. En medios libres se tienen, el agua en las corrientes marinas o en ríos y canales, así como el aire que circula en la atmósfera; cabe señalar que en flujos océano-atmosféricos, mucho más complicados y de gran importancia para efectos climáticos, la aproximación de Boussinesq juega un papel importante para simplificar el modelo, preservando aún así una buena aproximación del mismo, Lions et al. [6] y [7].

Entre los múltiples aspectos de estudio que presentan los flujos por convección natural, en ambos medios, existe un particular e importante interés tanto en la evolución del flujo mismo como en la transferencia de calor, y por lo tanto en los parámetros que tienen influencia sobre ellos. Esto se debe a la variedad de sus aplicaciones en diversas ramas de la ingeniería, la industria y la ciencia. Ejemplos de estas aplicaciones, en medios libres son: el enfriamiento de dispositivos electrónicos, aislamiento de edificios, intercambiadores de calor, aislamiento térmico de reactores nucleares, y aplicaciones de seguridad (transferencia de calor debida a incendios); mientras que en medios porosos se pueden mencionar: el almacenamiento y conservación de granos y cereales, colectores de energía solar, filtración y transporte de desechos radiactivos en el subsuelo y explotación eficiente de yacimientos de hidrocarburos y/o de mantos acuíferos. Por estas razones, resulta de gran interés el análisis y comprensión de la mecánica asociada con este tipo de sistemas, donde la información y conclusiones obtenidas pueden ser de gran valor práctico para la toma de decisiones en un problema determinado.

Matemáticamente, este tipo de flujos se modelan por las ecuaciones de Navier-Stokes en medios libres y por las de Darcy en medios porosos, acopladas en ambos casos con la ecuación de energía térmica, a través de la aproximación de Boussinesq. Las ecuaciones de Darcy son consecuencia de un proceso experimental y del modelo físico de conservación de masa, las de NavierStokes son consecuencia de los modelos físicos de conservación de momento y también del de conservación de masa. Originalmente dichas ecuaciones están formuladas en términos de las variables primitivas, velocidad y presión. 
Si bien las ecuaciones correspondientes en medios porosos son menos complejas que las de medios libres, en ambos casos se presentan serios inconvenientes para un adecuado tratamiento de las mismas. Por ello muchos estudios realizados en este sentido han reformulado los problemas respectivos en términos de las llamadas variables secundarias, función de corriente y vorticidad, con el objeto de evitar algunos de estos obstáculos; sin embargo, si bien algunos de ellos se eliminan, aparecen otros inconvenientes o limitaciones. En ambos casos, variables primitivas y secundarias, el acoplamiento con la ecuación de energía introduce la no linealidad en el sistema acoplado con las ecuaciones de Darcy y un efecto no lineal adicional en las ecuaciones de Navier-Stokes, análogo al que ya aparece en la ecuación de momento. Estas características dificultan el proceso para obtener soluciones aproximadas mediante un método numérico, lo cual no es más que un reflejo de las dificultades teóricas para demostrar que los sistemas continuos están bien planteados; como sucede por ejemplo en el caso isotérmico, ver Temam [8] y Chen y Ewing [9], o como en el caso evolutivo compresible en medios libres, ver Pironneau [10].

Diversos estudios realizados en ambos medios y con cualesquiera de las formulaciones mencionadas, se han encaminado a buscar y desarrollar métodos para enfrentar de manera adecuada las dificultades inherentes en cada caso, y poder obtener soluciones aproximadas confiables de manera eficiente. Sin embargo, no obstante que el desarrollo de mejores computadoras digitales permite analizar con mayor detalle los efectos no lineales y los fenómenos de acoplamiento, aún son muchas las dificultades para abordar eficientemente el estudio de esta clase de problemas, principalmente en lo concerniente a valores críticos de los parámetros que determinan la evolución del flujo así como la mayor o menor transferencia de calor (a través del número de Nusselt, local $N u$ o global $\overline{N u}$ ). Dichos parámetros son: el número de Rayleigh $R a$ ( $R a_{l}$ para medios libres, $R a_{p}$ para medios porosos); la relación entre las dimensiones de la región donde se desarrolla el flujo, por ejemplo la razón geométrica $A$ de una cavidad rectangular ( $A=$ cociente entre la altura y el ancho), y su ángulo de inclinación $\phi$. La magnitud de estos parámetros repercute directamente en las aplicaciones, por ejemplo, adicionalmente al efecto del número de Rayleigh: las dimensiones y el ángulo de inclinación de un colector de energía solar pueden conducir a la "recolección óptima" de energía para calefacción y/o generación de electricidad, en medios porosos; para lograr ambientes climáticos confortables (óptimos) en áticos, así como la inclinación óptima de alambiques solares para desalinizar agua y de desti- 
ladores para separar un líquido de otra sustancia, en medios libres. En el caso isotérmico la evolución de los flujos depende principalmente del parámetro dado por el número de Reynolds Re pero también de la razón geométrica $A$; al respecto, estudios recientes en variables secundarias, Goyon [11] y Nicolás y Bermúdez [12], y este trabajo mismo en variables primitivas, muestran que la evolución del flujo tiende a "complicarse" en la medida que $A$ aumenta, influencia que no ha sido muy estudiada hasta ahora. A continuación describimos brevemente algunos estudios desarrollados, en medios libres y porosos, en términos de variables primitivas y secundarias en regiones principalmente de dos dimensiones.

\section{Medios Libres:}

Bénard en 1900 y Rayleigh en 1916 fueron los pioneros en el estudio de lo que se conoce como convección de Bénard y que forma parte de los cimientos de la llamada convección natural, cuyas características y dinámica se han ido conociendo a partir de diversas investigaciones realizadas en esta dirección. Henkes y Hoogendoorn [13] presentan resultados del problema estacionario, en variables primitivas, en una cavidad cuadrada cuando $R a_{l}$ se incrementa en el rango $10^{3} \leq R a_{l} \leq 10^{8}$. Considerando el problema noestacionario: Hamady et al. [14] hacen una extensión a tres dimensiones a partir de un esquema en variables secundarias, con objeto de estudiar el efecto que tiene la inclinación de una cavidad tridimensional con sección transversal cuadrada sobre la transferencia de calor, para un rango del número de Rayleigh de $10^{4} \leq R a_{l} \leq 10^{6}$; Bermúdez y Nicolás [15] utilizan un esquema de descomposición de operadores en variables primitivas y reportan flujos con $10^{5} \leq R a_{l} \leq 10^{8}$ dentro de una cavidad cuadrada; Ding et al. [16] aplican un método en términos de variables secundarias en el cual combinan la expansión en series de Taylor con la aproximación de mínimos cuadrados ponderados para estudiar el flujo por convección natural en una cavidad cuadrada para números de Rayleigh $10^{4}$ y $10^{5}$. Por otra parte, flujos en cavidades bidimensionales altas (razón geométrica $A=8$ ) y con temperaturas prescritas en las paredes verticales, con una adimensionalización adecuada, han sido estudiados por algunos autores para el problema no-estacionario en variables primitivas. Christon et al. [17], en el año 2000, parecen ser los primeros en establecer las preguntas guía acerca del comportamiento del fluido en estas cavidades. En esta dirección, para el valor específico de $R a_{l}=3.4 \times 10^{5}$, podemos mencionar los trabajos: Xin y Le Quéré [18] estudiaron flujos dependientes del tiempo, considerando también el caso en que $R a_{l}=5.5 \times 10^{5}$, y 
determinaron los primeros puntos de bifurcación para los flujos e investigaron sobre la estabilidad de las soluciones; Auteri y Parolini [19] investigaron las primeras inestabilidades y reportan un fenómeno de ruptura de simetría, la cual se restaura posteriormente, aportando los primeros pasos en la transición al caos; en Glowinski [20], se reportan resultados a diferentes tiempos, lo cual parece indicar que el flujo es dependiente del tiempo.

Para el caso particular de flujos isotérmicos, ecuaciones de Navier-Stokes, varias de las investigaciones realizadas emplean, como problema de prueba, el caso de un fluido confinado en una cavidad cuadrada con tapa superior deslizable, lo cual origina el fenómeno de recirculación, con el objeto de validar o verificar la eficiencia del método propuesto y luego experimentar para valores de los parámetros que conducen a flujos más complicados; la solución de este problema no es trivial para números de Reynolds Re grandes ya que para capturar la dinámica rápida del fenómeno, debida a la viscosidad pequeña, se requiere de mallas finas y pasos de tiempo pequeños; como ya. se ha mencionado, algo análogo ocurre cuando se consideran cavidades con razón geométrica $A$ grande. A continuación, se resumen algunos trabajos desarrollados en esta dirección.

1) Problema no-estacionario en variables primitivas: Karniadakis et al. [21] proporcionan un método de proyección que se basa en una descomposición de operadores, en donde hacen uso de una combinación de reglas de integración explícitas e implícitas en la discretización temporal del problema no estacionario combinados con un método espectral para la discretización espacial, obteniendo esquemas de alto orden de precisión en el tiempo; el proceso requiere, en uno de sus pasos, resolver una ecuación de tipo Poisson para la presión y por lo tanto debe proponerse una condición de frontera adecuada; en base a algunos trabajos previos, Orzag et al. [22] y Gresho y Sani [23], se considera una condición de frontera de tipo Neumann; los resultados que se reportan corresponden a números de Reynolds bajos. Weinan y Liu [24] estudian la convergencia de un método de proyección y el efecto que tiene la elección de diferentes condiciones de frontera para la presión. Pan y Glowinski [25] con un método de descomposición de operadores de primer orden para la discretización temporal, combinan resolvedores de Stokes à la Uzawa con la solución de una ecuación de tipo onda para el término advectivo, y presentan resultados para la cavidad cuadrada considerando, primero, que la pared superior es la única que se desliza, y posteriormente que la pared inferior también lo hace. Estos deslizamientos son "suavizados", a diferencia de la condición de frontera "no regularizada" utilizada en este trabajo. 
Los flujos que obtienen convergen al estado estacionario para $R e \leq 7500$, en el primer caso, y para $R e \leq 4000$, en el segundo; mientras que un flujo periódico se obtiene en cada caso para $R e=8500$ y $R e=5000$, respectivamente. A través de métodos implícitos de volumen finito Sahin y Owens [26] evitan las dificultades asociadas con la presión eliminándola al tomar el producto vectorial de la ecuación de momento con el vector unitario normal, para un volumen de control en la frontera y después integrando alrededor de ella, de esta manera las ecuaciones que obtienen se expresan únicamente en términos de las componentes de la velocidad; este método lo aplican a problemas estacionarios y no estacionarios para $R e \leq 10000$, incluyendo resultados transitorios para $T \leq 12$. 2) Problema no-estacionario en variables secundarias: con un método de variables incrementales Goyon [11] obtiene resultados para $100 \leq R e \leq 7500$ que convergen al estado estacionario, un flujo periódico para $R e=12500$, y algunos resultados con $A=2$ para $100 \leq R e \leq 1000$. Por otra lado, Nicolás y Bermúdez [12] reportan el caso para $R e=1000$ con $A=2$ y 3 , así como una extensión para convección mixta de problemas térmicos. En este trabajo, dentro del contexto de variables primitivas, se reportan resultados para $R e=1000$ con $1 \leq A \leq 4$ y para $400 \leq R e \leq 15000$ con $A=1$.

Diversos trabajos, como los descritos anteriormente, estudian los flujos de convección natural e isotérmicos empleando una variedad de técnicas numéricas, sin embargo, generalmente consideran el efecto de variar sólo uno, o a lo más dos, de los siguientes parámetros: el número de Rayleigh $R a_{l}$ o de Reynolds $R e$, la razón geométrica $A$ o el ángulo de inclinación $\phi$ de la cavidad. En este sentido, los resultados obtenidos en cada uno de ellos, indican que los flujos se vuelven más complicados al incrementar alguno de estos parámetros, principalmente $A \mathrm{y}, R a_{l}$ en convección natural o $R e$ en flujos isotérmicos.

\section{Medios Porosos:}

Algunos de los primeros autores en tratar de analizar el flujo de convección natural en medios porosos, así como los mecanismos que influyen sobre ella, fueron Horton y Rogers en 1945 y Lapwood en 1948, por esta razón se le conoce como convección de Lapwood y se considera que es el análogo al problema de convección de Bénard. A partir de estos estudios y motivados en gran parte por las aplicaciones que tiene este tipo de convección en diversas áreas del conocimiento humano, muchos investigadores continúan estudiando y analizando una variedad de problemas relaciona- 
dos con ella, fundamentalmente en variables secundarias. 1) problema no estacionario: Holst y Aziz [27] introducen un vector potencial (contraparte tridimensional de la función de corriente) y aplican un esquema numérico de diferencias finitas para estudiar el flujo de convección natural, concluyendo que bajo ciertas condiciones y para un valor fijo del número de Rayleigh, el caso tridimensional genera mayor transferencia de calor que el correspondiente problema en dos dimensiones. Caltagirone y Bories [28] hacen uso de una teoría de estabilidad lineal para determinar las condiciones para la existencia y estabilidad de flujos de convección natural en un medio poroso inclinado, así como la influencia de la condición inicial para llegar al estado estacionario. Baytas [29] obtiene resultados del estado estacionario en una cavidad cuadrada con inclinación para $10^{2} \leq R a_{p} \leq 10^{4}$; para estos mismos números de Rayleigh, Saeid y Pop [30] estudian la evolución transitoria del fluido en una cavidad cuadrada, reportando el tiempo final en el que se alcanza el estado estacionario. 2) Problema estacionario: Moya et al. [31] aplicando el método del falso transitorio, investigan la convección natural en cavidades rectangulares inclinadas con números de Rayleigh $R a_{p} \leq 10^{2}$, encontrando flujo multicelular para algunos ángulos de inclinación $\phi$. Sen et al. [32] estudian la multiplicidad de soluciones, considerando cavidades inclinadas verticales y horizontales, mostrando resultados numéricos analíticos y numéricos para ángulos pequeños y $R a_{p} \leq 500$. Si bien en algunos de estos trabajos se considera el efecto de variar al menos dos de los parámetros $R a_{p}$, $A \circ \phi$, también puede apreciarse que esta variación es más restrictiva para $R a_{p}$, pues se reportan resultados sólo hasta valores de $R a_{p} \leq 10^{4}$.

A continuación, se describen las contribuciones y características del presente trabajo.

A diferencia de otros métodos que están diseñados para cada medio, poroso o libre, un aspecto novedoso de los métodos numéricos que se presentan aquí consiste en tener una estructura común para ambos medios, tanto en variables primitivas como en secundarias, y con excepción de éste último, que está limitado al caso de regiones rectangulares bidimensionales debido, entre otras razones, a la forma de construir la condición de frontera para la vorticidad, el de variables primitivas es independiente de la dimensión espacial y de la forma geométrica de la región que se considere, además no requiere de iteración alguna. En todos los casos se considera el problema no-estacionario con lo cual pueden estudiarse flujos que tienden a un estado estacionario, así como también los que puedan tener un compor- 
tamiento dependiente del tiempo (flujos oscilatorios). Una ventaja adicional es que los métodos numéricos en medios libres pueden adaptarse para estudiar flujos térmicos más complicados, por ejemplo flujos de convección mixta donde además del mecanismo térmico del gradiente de temperatura, se considera un mecanismo de movimiento, a través de la condición de frontera de la velocidad del sistema, Nicolás y Bermúdez [12] y [15]. Después de una discretización adecuada en tiempo, y de aplicar un proceso iterativo en el caso de variables secundarias, finalmente se tienen que resolver problemas elípticos lineales, simétricos y desacoplados para los que existen resolvedores eficientes, independientemente de la discretización espacial que se use.

Específicamente, respecto a los resultados numéricos se pueden señalar las siguientes contribuciones: 1) Para todos los resultados que convergen a un estado estacionario se reporta el tiempo $t_{\text {est }}$ en el cual éste se alcanza, un aspecto que usualmente no se reporta cuando se resuelve el problema no estacionario. En nuestro caso $T_{\text {est }}$ se determina, en general, mediante la norma discreta absoluta $L_{\infty}$ en la cerradura de la región de flujo, Nicolás y Bermúdez [12], lo cual no es más que la condición matemática discreta para medir el concepto físico del tiempo a partir del cual la solución ya no cambia en todo punto espacial, Landau [4] y Foias et al. [33]. Este valor de $T_{\text {est }}$ puede ayudar a determinar qué tan activo es un flujo, es decir qué tan vigoroso es su movimiento, dependiendo del valor de los parámetros involucrados. Por ejemplo, para flujos isotérmicos en la cavidad cuadrada, gobernados por las ecuaciones de Navier-Stokes, $T_{\text {est }}$ aumenta cuando Re se incrementa, como es de esperarse debido a que la dinámica del flujo es más rápida a medida que la viscosidad disminuye; al menos para $R e=1000$, se observa que $T_{\text {est }}$ también aumenta cuando $A$ aumenta. Sin embargo, para flujos térmicos en ambos medios, se observa que: al fijar $A$, el valor de $T_{e s t}$ disminuye cuando $R a$ se incrementa, mientras que cuando $R a$ es fijo, $T_{\text {est }}$ aumenta al incrementar $A$; por su parte, el ángulo de inclinación $\phi$ de la cavidad no ejerce un efecto tan sencillo sobre $T_{\text {est }}$ como los parámetros anteriores. Por otro lado, los pocos autores que hasta ahora hacen alguna alusión sobre el criterio de convergencia para alcanzar el estado estacionario, o dicho criterio no es claro o se menciona el error relativo discreto entre los niveles de tiempo actual y anterior, como en Goyon [11] y Pan y Glowinski [25]. 2) En relación a $T_{\text {est }}$ se concluye que el flujo isotérmico para $R e=10000$ es dependiente del tiempo ya que a diferencia de los otros con $R e$ menor éste no alcanza el estado estacionario, razón por lo cual se reporta la evolución del flujo a tiempos suficientemente grandes, donde pueden apreciarse varios aspectos o características propias 
de un flujo oscilatorio, además realizamos una comparación con otro flujo con $R e$ mayor, al menos a ciertos tiempos específicos. Debe tomarse en cuenta que para dichos flujos con $R e$ mayor el carácter oscilatorio del flujo tiende a ser cada vez más complejo, por su transición hacia la turbulencia, Landau [4] y Mohammadi y Pironneau [34]. Respecto a los resultados que se reportan para flujos isotérmicos con $R e=1000$ y razón geométrica $A>2$, éstos se obtuvieron empleando el error relativo, a diferencia de los otros casos, ya que el flujo cambia muy poco entre un paso de tiempo y otro; por esta razón empleamos, para este caso, un criterio de convergencia más fino. En este sentido, debido a la influencia de $A$, dichos flujos son más complicados. 3) En relación a los valores del número de Nusselt global $\overline{N u}$ y del mínimo de la función de corriente $\psi_{\min }$ se observa en ambos medios, libres o porosos, que al dejar fijo el número de Rayleigh $R a$ e incrementar la razón geométrica $A$, el flujo aumenta su actividad y existe mayor transferencia de calor, pero $T_{\text {est }}$ disminuye. Sin embargo, cuando $R a$ es fijo, el fluido es más activo pero la transferencia de calor es menor a medida que $A$ aumenta, y se requiere de más tiempo para que el flujo alcance el estado estacionario. 4) Seguimiento de la evolución del flujo en su etapa transitoria antes de llegar a su estado estacionario, o en diferentes estadíos para flujos dependientes del tiempo para medios libres. 5) Los resultados que se reportan para ambos medios, involucran, para ciertos valores de los parámetros, una parte de validación de los métodos al compararlos con resultados reportados por otros autores, obtenidos con esquemas diferentes, que se supone son correctos; después se reportan resultados que van más allá del proceso de validación explorando la robustez del método, llevando a cabo, en algunos casos, un estudio de independencia de mallas y del paso de tiempo, usando el correspondiente error relativo de la norma $L_{\infty}$, cuando el flujo se supone que se está reportando por primera vez. En la parte de validación, las mallas (uniformes en toda la región de flujo) y los pasos de tiempo, en la mayoría de los casos, son considerablemente grandes comparados con los empleados por otros autores, lo cual también se extiende a los casos más complicados. Tomando en cuenta que en el caso de variables primitivas no se itera, es de notarse lo competivo de los métodos, computacionalmente hablando.

Hacemos notar que en este trabajo no se consideran aspectos teóricos de convergencia y estabilidad de los métodos sino solamente la presentación de los flujos, con la consecuente parte de validación, haciendo énfasis en la eficiencia computacional del método y observando la congruencia física a través del flujo en sí o con el pos-procesamiento de la transferencia de calor median- 
te los números de Nusselt en los casos de convección natural; sin embargo, la congruencia que muestra el tamaño de los parámetros de la discretización espacial y temporal, que deben usarse a medida que el flujo tiende a ser más complejo, representa de alguna manera los efectos de convergencia y estabilidad de los métodos.

Finalmente, mencionamos que varios resultados en variables secundarias se han reportado en los trabajos Bermúdez et al. [35], Báez y Nicolás [36] y [37]. A continuación se describe la forma en que se desarrolla el trabajo de aquí en adelante.

En el Capítulo 2, se señalan las leyes físicas y las ecuaciones respectivas a que dan origen tanto en medios libres como en medios porosos. Se describe la aproximación de Boussinesq y la simplificación que se logra a través de ella en los sistemas obtenidos, los cuales posteriormente se procede a adimensionalizar; se señalan también algunas características de los parámetros adimensionales obtenidos y se describe el fenómeno de convección natural. En el Capítulo 3, se describen los esquemas numéricos para resolver los correspondientes sistemas, en ambos medios, y se presentan resultados que se obtienen al aplicar estos esquemas en cavidades rectangulares para algunos valores de los parámetros $R a$ y $A$. En el Capítulo 4, los sistemas adimensionales obtenidos en el Capítulo 2 son reformulados en términos de las variables secundarias, señalando las ventajas e inconvenientes de considerar esta nueva formulación. Se presentan los procedimientos numéricos para resolver los sistemas de ecuaciones en ambos medios y se muestran resultados para el caso de cavidades bidimensionales inclinadas con diversos valores de los parámetros. En el Capítulo 5, se presentan las conclusiones del trabajo. 


\section{Capítulo 2}

\section{Planteamiento del problema}

En este capítulo se describen las ecuaciones que modelan el flujo de fluidos en medios libres y en medios porosos sujetos a la aproximación de Boussinesq, y se obtiene la correspondiente adimensionalización de las mismas. Se describe con mayor detalle el fenómeno de convección natural, así como algunos de los parámetros obtenidos de la adimensionalización.

\subsection{Relaciones básicas de la mecánica de flui- dos en medios libres y medios porosos}

El flujo de un fluido viscoso, no estacionario y sujeto a efectos térmicos puede describirse en términos matemáticos a través de diversas ecuaciones, al apoyarse en varias leyes físicas teóricas y/o empíricas, que se preservan sobre el sistema dependiendo de sus características. Algunas de estas leyes y las respectivas ecuaciones a que dan origen son:

$\begin{array}{ll}\text { ley de conservación de la materia } & \rightarrow \text { ecuación de continuidad } \\ \text { primera ley de la termodinámica } & \rightarrow \text { ecuación energía } \\ \text { segunda ley de Newton } & \rightarrow \text { ecuación de momento } \\ & \rightarrow \text { (medios libres) } \\ \text { ley empírica de Darcy } & \rightarrow \text { ecuación de momento } \\ & \text { (medios porosos) }\end{array}$

La ley de conservación de la materia establece que la masa de un fluido en una región cerrada no se crea ni se destruye; la ecuación correspondiente se obtiene al aplicar un balance de masa a un volumen de control elemental 
a través del cual circula el fluido y considerando que la variación de acumulación de masa en el tiempo es igual a la diferencia entre la masa que entra y la que sale, a partir de lo cual se obtiene

$$
\frac{\partial \rho}{\partial t}+\nabla \cdot(\rho \mathbf{v})=0
$$

la cual se conoce como ecuación de continuidad, en donde $\rho$ representa la densidad, $t$ el tiempo y $\mathbf{v}$ la velocidad del fluido. En medios porosos, la ecuación de continuidad cambia ligeramente y está dada como

$$
\varphi \frac{\partial \rho}{\partial t}+\nabla:(\rho \mathbf{v})=s
$$

donde $\varphi$ es la porosidad del medio y $s$ representa, hablando compresiblemente, una distribución de fuentes y sumideros $(s=0$ en silos y calefactores solares; sin embargo, un ejemplo donde no necesariamente es cero es cuando representa la razón volumétrica del flujo en pozos de inyección o de extracción en recuperación, secundaria y terciaria, de petróleo). Si bien la ecuación de continuidad, para cada medio, está dada en forma general y es aplicable a fluidos cuya densidad varía con el tiempo y espacialmente de un punto a otro, existe una buena cantidad de éstos cuya densidad puede considerarse constante, esto es, no existe compresión o expansión notoria del fluido, es entonces que se habla de fluidos incompresibles. En tal caso la ecuación de continuidad en cualquiera de los dos casos, y considerando $s=0$ en medios porosos, se reduce a

$$
\nabla \cdot \mathbf{v}=0
$$

De manera análoga, la primera ley de la termodinámica expresa que la energía total de un sistema y sus alrededores permanece constante. La ecuación de energía, expresada en términos de la temperatura, describe el balance de calor en cada elemento de volumen, es decir, la rapidez neta con la que se genera calor en este volumen de control debido a todos los efectos de transporte de calor tales como conducción, convección y los debidos a reacciones químicas, Landau [4], Mohammadi y Pironneau [34] y Griebel et al. [38]. De esta manera, la ecuación de energía térmica está dada por

$$
c_{p} \rho\left(\frac{\partial T}{\partial t}+\mathbf{v} \cdot \nabla T\right)=\nabla \cdot(\eta \nabla T)-P \nabla \cdot \mathbf{v}+\Phi+Q
$$

donde $T$ representa la temperatura, $P$ la presión, $c_{p}$ el calor específico a presión constante, y $\eta$ la conductividad térmica del medio, mientras que $\Phi$ 
es la función de disipación viscosa, una medida de la rapidez con la que la energía mecánica se convierte en energía térmica. $Q$ indica la energía térmica generada por reacciones químicas.

Si bien la ecuación de continuidad y las características termodinámicas de un fluido deben conservarse en cualquier sistema hidrodinámico, independientemente del medio en el que se encuentre el fluido, la dinámica del mismo depende estrechamente del medio en el que se mueve. Por ello la ecuación de momento que describe dicha dinámica adquiere una forma distinta en medios libres y en medios porosos; en el caso de medios libres dicha ecuación es descrita por la segunda ley de Newton, la cual indica que la rapidez de cambio del momento lineal de un sistema es igual a la suma de todas las fuerzas que actúan sobre el mismo. Así, la ecuación de momento en medios libres está dada por

$$
\rho\left(\frac{\partial \mathbf{v}}{\partial t}+(\mathbf{v} \cdot \nabla) \mathbf{v}\right)=\nabla \cdot\left[\mu\left(\nabla \mathbf{v}+\nabla \mathbf{v}^{T}\right)-\frac{2 \mu}{3} \mathbf{I} \nabla \cdot \mathbf{v}\right]-\nabla P+\mathbf{F}
$$

en donde $\mu$ es la viscosidad dinámica del fluido y $\mathbf{F}$ representa las fuerzas externas que actúan sobre el mismo. La viscosidad $\mu$, al igual que otras propiedades del fluido, es función de P y T, Landau [4] y Mohammadi y Pironneau [34]. Si la viscosidad fuera constante entonces los términos dentro de los paréntesis del lado derecho de la ecuación (2.5) se reducirían a $\mu \nabla^{2} \mathbf{v}-$ $\frac{\mu}{3} \nabla(\nabla \cdot \mathbf{v})$.

Por otro lado, en medios porosos la dinámica de un fluido que pasa a través de los canales finos (poros) del medio, desde el punto de vista macroscópico, aparece en forma bastante diferente y se representa a partir de la ley experimental de Darcy en la cual se establece una relación entre las magnitudes fundamentales del flujo, la velocidad $\mathbf{v}$ y la presión $P$; entonces, la ecuación de momento está dada por

$$
\mathbf{v}=-\frac{\mathbf{K}}{\mu}(\nabla P-\mathbf{F}),
$$

donde $K$ representa la permeabilidad del medio poroso; en general se representa por una matriz y puede variar de punto a punto, si el medio es isotrópico entonces $K$ se considera constante en cualquier dirección y es entonces un escalar, Bear [5]. Aunque la ecuación de Darcy también involucra el término de la derivada temporal de la velocidad, éste puede eliminarse debido a que su contribución es despreciable comparado con el término $\frac{\mu}{K} \mathbf{v}$. Esta suposición 
es válida si la permeabilidad del medio poroso es pequeña, lo que a su vez conduce a que la velocidad $\mathbf{v}$ del fluido a través de los poros será igualmente pequeña, Tilton y Cortelezzi [39] y Whitaker [40].

Para completar el sistema de ecuaciones, en cada medio, respecto al número de incógnitas, es necesario otra relación la cual puede estar dada por la ecuación de estado que en general representa el cambio de la densidad del fluido cuando varía la presión y/o la temperatura del mismo, esto es $\rho=\rho(P, T)$.

Así, el flujo de un fluido no estacionario, térmico, compresible, y viscoso, sujeto a una fuerza externa gravitacional $\mathbf{F}=\rho \mathbf{g}$, en donde no se presentan reacciones químicas, se desprecia la disipación viscosa $(\Phi=0)$, no existen fuentes o sumideros, y además se considera la ecuación de estado respectiva $\rho=\rho(P, T)$, puede ser descrito matemáticamente, en uno y otro medio, como

\section{Medios Libres}

$$
\begin{array}{ll}
\frac{\partial \rho}{\partial t}+\nabla \cdot(\rho \mathbf{v}) & =0 \\
c_{p} \rho\left(\frac{\partial T}{\partial t}+\mathbf{v} \cdot \nabla T\right) & =\nabla \cdot(\eta \nabla T)-P \nabla \cdot \mathbf{v} \\
\rho\left(\frac{\partial \mathbf{v}}{\partial t}+(\mathbf{v} \cdot \nabla) \mathbf{v}\right) & =\nabla \cdot\left[\mu\left(\nabla \mathbf{v}+\nabla \mathbf{v}^{T}\right)-\frac{2 \mu}{3} \mathrm{I} \nabla \cdot \mathbf{v}\right]-\nabla P+\rho \mathbf{g}
\end{array}
$$

\section{Medios Porosos}

$$
\begin{array}{ll}
\varphi \frac{\partial \rho}{\partial t}+\nabla \cdot(\rho \mathbf{v}) & =0 \\
c_{p} \rho\left(\frac{\partial T}{\partial t}+\mathbf{v} \cdot \nabla T\right) & =\nabla \cdot(\eta \nabla T)-P \nabla \cdot \mathbf{v} \\
\mathbf{v}=-\frac{\mathbf{K}}{\mu}(\nabla P-\rho \mathbf{g}) . &
\end{array}
$$

\subsection{Aproximación de Boussinesq}

Pueden existir diversas fuerzas actuando sobre un fluido, entre ellas la fuerza externa producida por el campo gravitacional terrestre; si existen gradientes de temperatura en el fluido, se producirán diferencias de densidad en el 
mismo, y en un campo gravitatorio, el fluido más caliente y menos denso ascenderá, mientras que el más frío y más denso descenderá. Este tipo de movimiento, debido exclusivamente a la no uniformidad de la temperatura del fluido, se denomina convección natural y es la responsable fundamental de transferir el calor de una parte del fluido a otra. En este proceso convectivo las moléculas en movimiento intercambian energía, originándose transferencia de calor en el fluido.

Cuando se describe matemáticamente el flujo de un fluido, en general compresible, incorporando estos efectos de la temperatura, lo que resulta es un sistema no lineal de ecuaciones que no es fácil de tratar, por esta razón se requiere hacer algunas simplificaciones. Una de estas simplificaciones es la aproximación de Boussinesq, la cual considera que la densidad es constante, excepto en el término $\rho \mathrm{g}$ de la fuerza de gravedad en la ecuación de momento, lo que a su vez implica la estructura incompresible del fluido $(\nabla \cdot \mathbf{v}=0)$, Griebel et al [38] y Gunzburguer [41].

Además, para la densidad $\rho=\rho(P, T)$ en el término $\rho \mathrm{g}$, se considera un desarrollo de Taylor alrededor de una temperatura de referencia $T_{o}$ hasta el término de primer orden, de esta forma se tiene $\rho=\rho_{o}\left[1-\beta\left(T-T_{o}\right)\right]$, donde $\beta=-\frac{1}{\rho_{0}} \frac{\partial \rho}{\partial T}$, lo que indica que se están considerando cambios pequeños de temperatura, respecto a $T_{o}$, y que los cambios de densidad respecto a cambios de presión se desprecian, por considerarse éstos muy pequeños. La influencia de la densidad en esta forma da origen a un efecto de flotabilidad en la fuerza de gravedad, llamada precisamente fuerza de flotación,

Puesto que se está considerando que las diferencias de temperatura son pequeñas, podemos despreciar la variación con la temperatura de la viscosidad $\mu$, de la conductividad térmica $\eta$, el coeficiente de expansión térmica $\beta$, así como del calor específico $c_{p}$ y suponerlas entonces constantes Griebel et al. [38].

Con estas suposiciones se obtiene entonces una estructura incompresible, conocida como aproximación de Boussinesq, que en términos matemáticos y dependiendo del medio en el que se encuentre el fluido adquiere la siguiente forma: 


\section{Medios Libres}

$$
\begin{array}{ll}
\nabla^{*} \cdot \mathbf{v} & =0 \\
\frac{\partial T}{\partial t^{*}}+\mathbf{v} \cdot \nabla^{*} T & =\kappa \nabla^{* 2} T \\
\rho_{o}\left(\frac{\partial \mathbf{v}}{\partial t^{*}}+\left(\mathbf{v} \cdot \nabla^{*}\right) \mathbf{v}\right) & =\mu \nabla^{* 2} \mathbf{v}-\nabla^{*} P+\rho_{o}\left[1-\beta\left(T-T_{0}\right)\right] \mathbf{g}
\end{array}
$$

\section{Medios Porosos}

$$
\begin{aligned}
& \nabla^{*} \cdot \mathbf{v}=0 \\
& \frac{\partial T}{\partial t^{*}}+\mathbf{v} \cdot \nabla^{*} T=\kappa \nabla^{* 2} T \\
& \mathbf{v}=-\frac{k}{\mu}\left(\nabla^{*} P-\rho_{o}\left[1-\beta\left(T-T_{o}\right)\right] \mathbf{g}\right)
\end{aligned}
$$

donde $\kappa=\frac{\eta}{c_{p} \rho_{0}}$, en la ecuación de energía para ambos medios, se conoce como el coeficiente de difusividad térmica, y el símbolo $\left({ }^{*}\right)$ hace referencia a las variables dimensionales.

De acuerdo a esta simplificación la ecuación de continuidad en ambos medios se reduce a $\nabla^{*} \cdot \mathbf{v}=0$, la cual también se conoce como condición de incompresibilidad. Por otra parte, si el fluido está confinado en una región $\Omega$ los sistemas de ecuaciones deben complementarse con condiciones iniciales y de frontera para tener un sistema bien planteado.

\subsection{Problemas Adimensionales}

Para poder estudiar y analizar los sistemas de ecuaciones descritos en la sección anterior, es conveniente obtener su representación adimensional. Para ello, hacemos uso de las siguientes variables adimensionales respectivas:

\section{Medios Libres}

$$
x=\frac{x^{*}}{L}, \quad y=\frac{y^{*}}{L}, \quad \theta=\frac{T-T_{0}}{T_{1}-T_{2}}, \quad t=\frac{t^{*} U_{0}}{L}, \quad \mathbf{u}=\frac{\mathbf{v}}{U_{0}}, \quad p=\frac{P+\rho_{0} g y^{*}}{\rho_{0} U_{0}^{2}},
$$

\section{Medios Porosos}

$$
x=\frac{x^{*}}{L}, \quad y=\frac{y^{*}}{L}, \quad \theta=\frac{T-T_{o}}{T_{1}-T_{2}}, \quad t=\frac{t^{*} \kappa}{L^{2}}, \quad \mathbf{u}=\frac{\mathbf{v} L}{\kappa}, \quad p=\frac{\left(P+\rho_{o} g y^{*}\right) k}{\mu \kappa},
$$


Las cantidades $L, T_{o}, T_{1}, T_{2}, \rho_{o}$ y $U_{o}$ que aparecen en los problemas son valores de referencia para la longitud, la temperatura, la densidad y la velocidad respectivamente.

Sustituyendo las variables dimensionales en los sistemas (2.9) y (2.10) por las respectivas adimensionales y simplificando, obtenemos los correspondientes sistemas de ecuaciones adimensionales que describen el flujo de fluidos, sujeto a la aproximación de Boussinesq, en ambos medios.

Sea $\Omega \subset R^{N}(N=2,3)$ la región donde se desarrolla el flujo de un fluido incompressible, viscoso, térmico y dependiente del tiempo, y sea $\Gamma$ la frontera de dicha región. Las ecuaciones matemáticas adimensionales que modelan dicho flujo en medios libres $(m l)$ y en medios porosos $(m p)$ están dadas por:

$$
\begin{aligned}
& (m l)\left\{\begin{array}{lll}
t>0: & =0 & \text { en } \Omega,(a) \\
\theta_{t}-\frac{1}{R e P r} \nabla^{2} \theta+\mathbf{u} \cdot \nabla \theta & =-\frac{R a_{l}}{P r R e^{2}} \theta \mathbf{e} & \text { en } \Omega, \quad(b) \\
\mathbf{u}_{t}-\frac{1}{R e} \nabla^{2} \mathbf{u}+\nabla p+(\mathbf{u} \cdot \nabla) \mathbf{u} & \text { en } \Omega . \quad(c) \\
\nabla \cdot \mathbf{u} & =0 &
\end{array}\right. \\
& (m p)\left\{\begin{array}{lll}
t>0: & \\
\theta_{t}-\nabla^{2} \theta+\mathbf{u} \cdot \nabla \theta & =0 & \text { en } \Omega, \quad(a) \\
\mathbf{u}+\nabla p & =-R a_{p} \theta \mathbf{e} & \text { en } \Omega, \quad(b) \\
\nabla \cdot \mathbf{u} & =0 & \text { en } \Omega . \quad(c)
\end{array}\right.
\end{aligned}
$$

donde las variables adimensionales $\mathbf{u}, p$ y $\theta$ representan la velocidad, la presión y la temperatura del flujo respectivamente, y e es el vector unitario en la dirección de la aceleración gravitacional. Los parámetros que se obtienen en el proceso de adimensionalización son: $\operatorname{Re}=\frac{U_{0} L}{\nu}, \operatorname{Pr}=\frac{\nu}{\kappa}$ y $R a_{l}=\frac{\beta L^{3} g\left(T_{1}-T_{2}\right)}{\kappa \nu}$, los cuales se conocen como los números de Reynolds, de Prandtl y de Rayleigh respectivamente en medios libres, mientras que $R a_{p}=\frac{k \beta L g\left(T_{1}-T_{2}\right)}{\kappa \nu}$ corresponde al número de Rayleigh en medios porosos; en ambos casos, $\nu=\frac{\mu}{\rho}$ representa la viscosidad cinemática.

Por otro lado, si en medios libres no existe contribución de la temperatura, la ecuación de energía se elimina del sistema (2.11) y el lado derecho 
de la ecuación de momento (2.11b) sería dado en términos de una fuerza externa, independiente de la temperatura, y de esta manera el sistema (2.11) se transforma en las ecuaciones de Navier-Stokes, que corresponden al caso isotérmico.

Es posible obtener un sistema adimensional alterno a (2.11), el cual se utiliza con frecuencia cuando se emplean cavidades rectangulares con razón geométrica A (razón entre el largo y ancho de la cavidad) grande, combinado con valores altos de $R a_{l}$. A partir de las mismas variables adimensionales y tomando: $U_{o}=\sqrt{g \beta L\left(T_{1}-T_{2}\right)}$ y $T_{o}=\frac{T_{1}+T_{2}}{2},[17]$, llegamos al siguiente sistema:

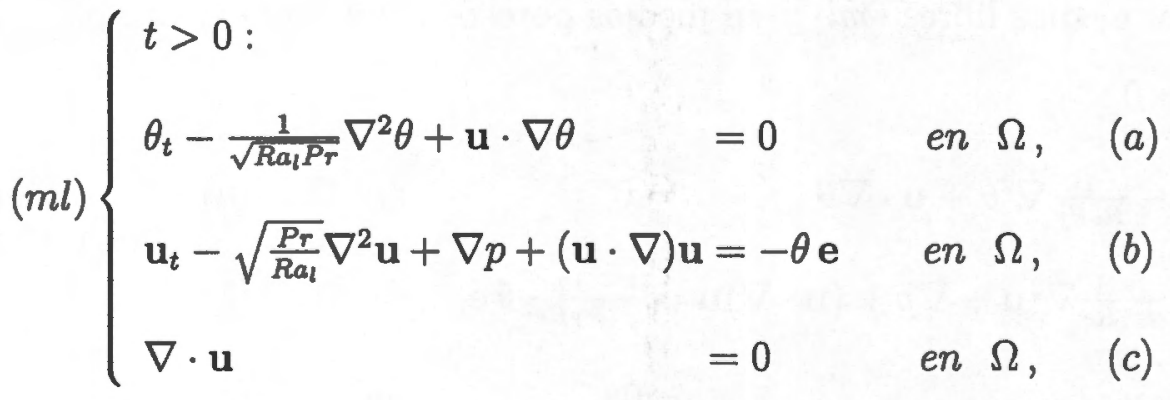

Como se mencionó anteriormente, el sistema de ecuaciones que modela el flujo de un fluido por convección natural, independientemente del medio, debe complementarse con condiciones iniciales y de frontera. Considerando que $\Omega$ es una región acotada, éstas condiciones pueden ser descritas como

i) condiciones iniciales:

$$
\left\{\begin{array}{lll}
\theta(\mathbf{x}, 0)=\theta_{o}(\mathbf{x}) & \text { en } \Omega \\
\mathbf{u}(\mathbf{x}, 0)=\mathbf{u}_{o}(\mathbf{x}) & \text { en } \Omega\left(\nabla \cdot \mathbf{u}_{o}=0\right) & (a)
\end{array}\right.
$$

ii) condiciones de frontera:

$$
\left\{\begin{array}{lll}
B \theta=0 & \text { sobre } \Gamma, t \geq 0 \\
\mathbf{u}=\mathbf{g} & \text { sobre } \Gamma, t \geq 0
\end{array}\left(\int_{\Gamma} \mathbf{g} \cdot \mathbf{n} d \Gamma=0\right), \quad(b)\right.
$$

donde $B$ es un operador de frontera para la temperatura el cual puede involucrar condiciones de frontera Dirichlet, Neumann o mixtas. 


\subsubsection{Parámetros adimensionales y convección natural}

Suponiendo que el fluido está confinado en una cavidad, por ejemplo rectangular, cuyas paredes tienen la misma temperatura, entonces en el estado de equilibrio el fluido alcanza esta misma temperatura y no presenta variación de ésta, ni de la densidad, ni de la presión, por lo cual se dice que es uniforme.

Cuando se aplica una ligera perturbación a la temperatura, el sistema regresará al estado de equilibrio tan pronto aquella cese. Si la cavidad tiene sus paredes inferior y superior a una misma temperatura, y se aumenta ligeramente la temperatura de la inferior, se generará un flujo contínuo de energía a través del fluido, transmitiendo el calor de la superficie caliente a la fría mediante una redistribución de la energía a nivel molecular. El fluido pierde su uniformidad; la temperatura, la densidad y la presión varían linealmente entre la superficie caliente y la fría. A este proceso se le llama conducción, no es una estructura auto-organizada y es impuesta por las condiciones externas.

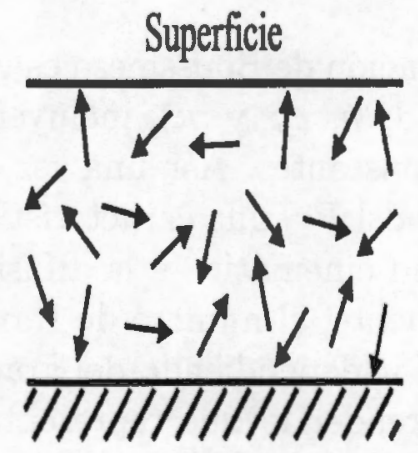

Superficie

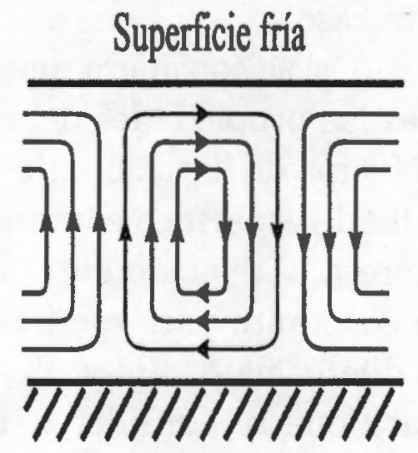

Superficie caliente

Fig. 2.1: Celdas convectivas

Si continuamos aumentando progresivamente la temperatura, llegará un momento en el cual el comportamiento del fluido cambia radicalmente, el movimiento aleatorio de las moléculas se ordena espontáneamente y aparecen celdas convectivas Fig. 2.1. Esta situación se presenta debido a que el fluido que se encuentra en contacto con la superficie caliente aumenta su temperatura por conducción, se expande y su densidad disminuye, con lo cual se generan diferencias de densidad en el fluido; en un campo gravitacional estas diferencias originan que el fluido más frío y denso tienda a irse al fondo, debido a la fuerza de gravedad, y empujar al fluido caliente y menos denso hacia arriba (fuerza de flotación), con esto se establece un movimiento, llamado 
flujo convectivo, que transmite el calor por todo el fluido, y en donde la fuerza de flotación está determinada por el gradiente de temperatura. De aquí que la convección natural no pueda darse si no existe una fuerza gravitacional.

Si la temperatura, el cual es el parámetro que regula la no linealidad del sistema, continúa en aumento, la estructura llega a ser más compleja en espacio y en tiempo, hasta alcanzar un régimen caótico o turbulento. Los sistemas capaces de exhibir auto-organización contienen los ingredientes de la dinámica caótica, y por ello exhiben estos regímenes, asociados a los distintos valores de los parámetros.

Entre estos parámetros, como se observa de los sistemas de ecuaciones adimensionales, se encuentran $R e, P r$, y $R a$ ( $=R a_{l}$ o $R a_{p}$ ), los cuales pueden tomar diversos valores. El número de Reynolds Re expresa la razón de las fuerzas inerciales a las viscosas y está asociado con el proceso de convección (natural o mixta). En convección natural no existe una velocidad $U_{o}$ de referencia, por lo que ésta se define como $U_{o}=\frac{\nu}{L}$, con lo que entonces $R e=1$ en este caso.

Por otro lado, si se considera que la aproximación de Boussinesq es válida, entonces todas las propiedades del fluido $\left(\mu, \beta, \eta, c_{p}\right.$ y $\left.\kappa\right)$, incluyendo $\rho$, excepto en la fuerza de flotación, permanecen constantes. Así, una vez que se considera un fluido específico el número de Prandtl $P r$, una característica del fluido que expresa la relación entre la viscosidad cinemática y la difusividad térmica, será constante para ese fluido. Finalmente, el número de Rayleigh, $R a_{l}$ o $R a_{p}$, a diferencia de $R e$ y $P r$, puede variar dependiendo del gradiente de temperatura que se considere. Para comprender lo que representa este parámetro a través de los elementos físicos que lo describen, a continuación hacemos un breve recuento del proceso histórico que condujo a su definición.

En 1900, Henri Bénard realizó experimentos calentando una capa delgada de fluido por abajo y observando que se obtenía un diagrama ordenado de celdas poligonales. Posteriormente, en 1916, Lord Rayleigh estudió e intentó explicar el origen de este patrón, desarrollando así una teoría lineal para explicar lo observado por Bénard y concluyó que los elementos que determinan que aparezca la convección son: la fuerza de flotación inducida por la presencia de un gradiente de temperatura, y los mecanismos que tienden a disipar la energía, esto es, la viscosidad, que ejerce fricción sobre la masa del fluido y trata de frenarlo y la difusividad térmica que se resiste al empuje tratando de anular la diferencia de temperaturas, Fig. 2.2. La convección inicia cuando la fuerza de flotación, que es la fuerza desestabilizadora, supera los efectos disipativos de la viscosidad y de la difusión del calor (que representan a la 
fuerza estabilizante). La relación entre estos tres mecanismos pueden combinarse en un sólo parámetro adimensional llamado número de Rayleigh, $R a$, a través del cociente entre el empuje y el producto de la viscosidad y la difusión térmica, el cual permite medir la influencia relativa entre ellos. Actualmente

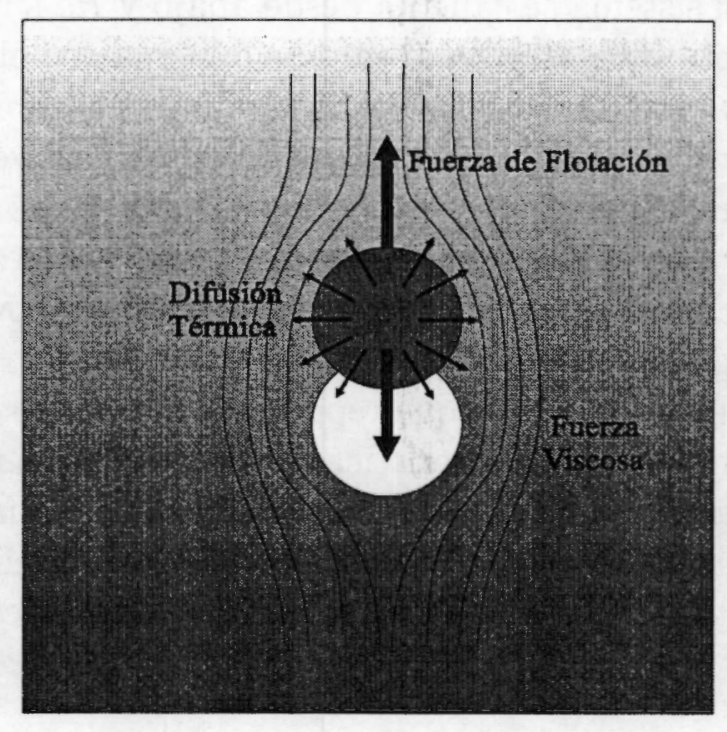

Fig. 2.2: Mecanismos involucrados en la convección

se sabe, sin embargo, que la teoría de Rayleigh realmente no es válida para explicar los experimentos de Bénard, ya que el movimiento del fluido y las estructuras formadas y observadas por éste último se deben a la variación de la tensión superficial generada por la temperatura, y a la inestabilidad provocada por un gradiente de densidades, el cual a su vez es producido por una diferencia de temperatura. Por lo tanto, las predicciones sobre convección que se desprenden de la teoría de Rayleigh sólo son válidas cuando $R a$ se encuentra cerca de un valor crítico, esto es, cuando apenas inicia el proceso convectivo. Aunque algunas teorías lineales posteriores eliminan algunos defectos que existen en la de Rayleigh, persiste la desventaja de que sólo tienen validez si la velocidad no es muy grande.

En base a lo anterior y por la expresión del número de Rayleigh $R a$ (= $R a_{l} \circ R a_{p}$ ), podemos afirmar entonces que cuando la fuerza de flotación, determinada por la diferencia de temperaturas $\left(T_{2}-T_{1}\right)$, supera a la disipación causada por la viscosidad $\nu$ y por la difusión térmica $\kappa$ se origina el movimiento convectivo, debido a que el sistema tiende a reducir su energía. 
Ante una perturbación, el sistema puede tender a alejarse de su estado original, o puede no alejarse ni acercarse nuevamente a él. Así, entre los valores que puede tomar $R a$, existirá un valor en el cual las fuerzas de flotación y disipativas se encontrarán en equilibrio neutro, a dicho valor se le conoce como $R a$ crítico. Si el sistema se calienta desde abajo y $R a$ supera este valor crítico, el fluido caliente sube, debido al empuje o fuerza de flotación, y el frío baja, por efecto de la gravedad, y como el fluido no puede subir y bajar en el mismo lugar, se genera un patrón de celdas convectivas a través de las cuales éste circula de manera alternada en el sentido de giro de las manecillas del reloj en unas celdas y en el sentido opuesto en las adyacentes. Este patrón convectivo es dinámico pues las moléculas permancerán en movimiento mientras esté presente el gradiente de temperatura.

Aunque este breve análisis de lo que representa el número de Rayleigh es válido para ambos medios, las características específicas de cada uno de ellos, en su expresión matemática dada en la sección anterior, varían ligeramente dependiendo del medio a que se hace referencia, razón por lo cual se distingue entre el número de Rayleigh en medios libres $R a_{l}$ y el respectivo en medios porosos $R a_{p}$.

\subsubsection{Transferencia de calor y Número de Nusselt}

La temperatura es una medida de la energía interna asociada al movimiento de las partículas de un fluido. Las partículas en movimiento chocan unas con otras, y la energía cinética se transfiere de una partícula con mayor velocidad a otra de velocidad menor, esto significa que la energía se transmite de una región con temperatura alta a otra con menor temperatura, así la mayor parte del calor es transportado de una región a otra por el mismo fluido. La capa del fluido que se encuentra en contacto con una superficie caliente recibe calor de ésta última por conducción, ya que no existe movimiento relativo del fluido en esta capa, y este calor pasa entonces al resto del fluido por convección.

La transferencia de calor por convección ocurre entre un fluido en movimiento y una superficie sólida, donde ésta última se encuentra a mayor temperatura que el primero. El número de Nusselt local $N u$ es una representación adimensional de la transferencia de calor por convección, esto es, permite determinar la capacidad para transferir calor por convección entre una superficie con temperatura prescrita y el fluido circundante. Se define en forma 
general como

$$
N u(s)=\frac{\partial \theta}{\partial \vec{n}}
$$

donde $\vec{n}$ indica la dirección normal unitaria a la superficie.

El flujo de calor promedio que se transfiere de la superficie al fluido se denomina número de Nusselt global $\overline{N u}$ y se calcula en la forma

$$
\overline{N u}=\frac{1}{L} \int_{0}^{L} N u(s) d s
$$

donde $L$ representa la longitud de la frontera o superficie.

En particular, si se considera una región rectangular donde se desarrolla el flujo, y la frontera caliente fuera la inferior, entonces sobre esa pared, los números de Nusselt, local y global, serían calculados respectivamente como

$$
\begin{gathered}
N u(x)=\left.\frac{\partial \theta}{\partial y}\right|_{y=0} \\
\overline{N u}=\frac{1}{L} \int_{0}^{L} N u(x) d x .
\end{gathered}
$$

Así, para evaluar el número de Nusselt, local o global, se requiere conocer el campo de temperatura en la región de estudio del fluido. 


\section{Capítulo 3}

\section{Esquemas numéricos en variables primitivas}

En este capítulo se presentan los esquemas numéricos correspondientes para aproximar la solución de los sistemas (2.11) en medios libres y (2.12) en medios porosos, en términos de la temperatura $\theta$ y de las variables primitivas, velocidad $\mathbf{v}$ y presión $p$. Ambos esquemas numéricos, como podrá observarse más adelante, tienen una estructura común.

Los procedimientos numéricos que se presentan aquí tienen la característica de que son independientes de la discretización espacial y, aunque sólo se presentan resultados para el caso bidimensional, también pueden aplicarse al caso tridimensional; no es necesario iterar lo cual, unido al hecho de que los resultados se obtienen con mallas significativamente gruesas, implica que los métodos sean bastante competitivos computacionalmente. Los resultados corresponden a valores del número de Rayleigh relativamente grandes (lo cual significa considerar lados derechos grandes en la ecuación de momento) en el caso de convección natural, y a valores grandes del número de Reynolds (advección dominante) para flujos isotérmicos en medios libres, en cavidades rectangulares $(A \geq 1)$. De manera específica, para medios libres consideramos $10^{5} \leq R a_{l} \leq 10^{7}$ en cavidades donde $1 \leq A \leq 3$, mientras que para flujos isotérmicos se consideran cavidades con tapa superior deslizable, lo cual se conoce como el problema de la cavidad con tapa deslizable, con valores del número de Reynolds $R e$ en el rango $400 \leq R e \leq 15000$ en la cavidad cuadrada, y $R e=1000$ para $1 \leq A \leq 4$. En medios porosos consideramos $10^{2} \leq R a_{p} \leq 10^{3}$ en cavidades cuadradas. 


\subsection{Esquema numérico en medios libres}

Tanto el problema térmico como el isotérmico, involucran dificultades inherentes a la no linealidad de la ecuación de momento y su acoplamiento con la condición de incompresibilidad; en este sentido varios autores han reportado diferentes enfoques para superar dicho acoplamiento, entre los cuales se encuentran los métodos de descomposición de operadores (o de paso fraccionado) que subdividen el problema en varios subproblemas más fáciles de resolver. A su vez, y de acuerdo a la forma en que se trata la presión, estos métodos se pueden clasificar en dos clases. En los métodos de la primera clase, se proporciona información adicional a través de una ecuación funcional que satisface la presión, con una formulación variacional dentro del marco de un espacio de Hilbert apropiado y que se resuelve con una técnica iterativa de gradiente conjugado, Bermúdez y Nicolás [15] y Bristeau et al. [42]. En los métodos de la segunda clase, se obtiene una ecuación de tipo Poisson para la presión, para lo cual se debe elegir una condición de frontera apropiada de tipo Neumann, Karniadakis et al. [21], y contrario a los métodos descritos anteriormente no requieren de técnicas iterativas para resolverse si adicionalmente, el término no lineal se aproxima explícitamente. En cualquiera de los dos casos, las dificultades asociadas con la no linealidad y con la condición de incompresibilidad se desacoplan. En esta sección se emplea un esquema que como se verá, cae dentro de la segunda clasificación y en el cual se hace uso del teorema de Helmholtz-Hodge, Foias et al. [33], el cual asegura que un campo vectorial $\mathbf{w}$ sobre un dominio $\mathrm{D}$ se puede descomponer de manera única en la forma $\mathbf{w}=\mathbf{u}+\nabla p$, donde $\nabla \cdot \mathbf{u}=0$ y $\mathbf{u} \cdot \mathbf{n}=0$ sobre la frontera de D.

En cada paso de tiempo, el proceso numérico resuelve, en primer lugar la ecuación de energía, y posteriormente las ecuaciones de momento y de continuidad mediante un método de descomposición de operadores a partir de una discretización temporal de segundo orden. En el primer paso de esta descomposición, obtenemos una velocidad intermedia, la cual no satisface la condición de incompresibilidad. Posteriormente, y empleando el teorema de Helmholtz-Hodge, proyectamos esta velocidad sobre el espacio de velocidades con divergencia cero para obtener la segunda velocidad intermedia. Para ello, debemos resolver primero una ecuación de tipo Poisson para la presión con su correspondiente condición de frontera Neumann. En el último paso, se obtiene la velocidad final del fluido.

El esquema para medios libres en su parte isotérmica es bastante cercano a 
uno ya reportado para flujos multifásicos en donde emplean una discretización espacial basada en métodos espectrales, Badalassi et al. [43], para manejar eficientemente dichos flujos que son más complicados que los monofásicos e isotérmicos reportados aquí. Los métodos espectrales no se usan aquí y por otro lado, el esquema que presentamos se ha extendido a flujos térmicos de convección natural y consideramos que sin mayor inconveniente puede extenderse al caso de convección mixta; mientras que el correspondiente a medios porosos hasta donde sabemos se está reportando por primera vez en este trabajo. Debido a que tenemos que resolver una ecuación de tipo Poisson para la presión, la respectiva condición de frontera de tipo Neumann se obtiene siguiendo un procedimiento similar al empleado por otros autores, Karniadakis et al. [21], Orzag et al. [22], Gresho y Sani [23], Badalassi et al. [43], tomando la componente normal, de la ecuación de momento semidiscreta, sobre la frontera. En este contexto, recientemente Sani et al. [44] muestran que se obtiene la misma solución al resolver la ecuación de momento acoplada ya sea considerando una ecuación de tipo Poisson para la presión con su respectiva condición de frontera Neumann, o si se considera, en su lugar, la condición de incompresibilidad.

En todos los modelos matemáticos empleados, tanto en variables primitivas como en variables secundarias, las derivadas temporales se aproximan a través del siguiente esquema de segundo orden

$$
f_{t}(\mathbf{x},(n+1) \Delta t) \approx \frac{\frac{3}{2} f^{n+1}-2 f^{n}+\frac{1}{2} f^{n-1}}{\Delta t}, \quad n \geq 1,
$$

donde $\Delta t(\geq 0)$ denota el paso de tiempo y $f^{k}$ es una aproximación para $f(\mathbf{x}, k \Delta t)$, con $f$ suficientemente suave.

Así el correspondiente sistema semidiscreto en tiempo para (2.11) toma la forma

$$
\begin{gathered}
\frac{\frac{3}{2} \theta^{n+1}-2 \theta^{n}+\frac{1}{2} \theta^{n-1}}{\Delta t}-\frac{1}{R e P r} \nabla^{2} \theta^{n+1}+\left(\mathbf{u}^{n+1} \cdot \nabla\right) \theta^{n+1}=0 \\
\frac{\frac{3}{2} \mathbf{u}^{n+1}-2 \mathbf{u}^{n}+\frac{1}{2} \mathbf{u}^{n-1}}{\Delta t}-\frac{1}{R e} \nabla^{2} \mathbf{u}^{n+1}+\left(\mathbf{u}^{n+1} \cdot \nabla\right) \mathbf{u}^{n+1} \\
+\nabla p^{n+1}=\frac{R a_{l}}{\operatorname{Pr} R e^{2}} \theta^{n+1} \mathbf{e}_{2} \\
\nabla \cdot \mathbf{u}^{n+1}=0
\end{gathered}
$$

donde $\mathbf{e}_{2}=-\mathbf{e}=(0,1)$. 
Los términos convectivos $\mathbf{u} \cdot \nabla \mathbf{u}$ y $\mathbf{u} \cdot \nabla \theta$ en cada nivel de tienpo $n+1$, se aproximan mediante una extrapolación lineal de los valores obtenidos en los niveles de tiempo $n$ y $n-1$, de esta manera tenemos

$$
\begin{aligned}
& (\mathbf{u} \cdot \nabla \mathbf{u})^{n+1}=2(\mathbf{u} \cdot \nabla \mathbf{u})^{n}-(\mathbf{u} \cdot \nabla \mathbf{u})^{n-1} \\
& (\mathbf{u} \cdot \nabla \theta)^{n+1}=2(\mathbf{u} \cdot \nabla \theta)^{n}-(\mathbf{u} \cdot \nabla \theta)^{n-1} .
\end{aligned}
$$

En el esquema numérico, en cada nivel de tiempo resolvemos, primero, la ecuación de energía para obtener $\theta$ y, posteriormente, en los siguientes tres pasos aplicamos un procedimiento de descomposición de operadores a las ecuaciones de momento y de continuidad para calcular y y $p$. Así, los pasos a seguir son

$$
\text { Paso 1) } \begin{gathered}
\frac{\frac{3}{\theta^{n+1}-2 \theta^{n}+\frac{1}{2} \theta^{n-1}}}{\Delta t}-\frac{1}{R e P P} \nabla^{2} \theta^{n+1}=-2\left(\mathbf{u}^{n} \cdot \nabla\right) \theta^{n} \\
+\left(\mathbf{u}^{n-1} \cdot \nabla\right) \theta^{n-1}
\end{gathered}
$$

Paso 2) $\quad \frac{\mathbf{u}^{*}-2 \mathbf{u}^{n}+\frac{1}{2} \mathbf{u}^{n-1}}{\Delta t}=-2\left(\mathbf{u}^{n} \cdot \nabla\right) \mathbf{u}^{n}+\left(\mathbf{u}^{n-1} \cdot \nabla\right) \mathbf{u}^{n-1}$$$
+\frac{R a_{1}}{P r R e^{2}} \theta^{n+1} \mathbf{e}_{2} \text { en } \Omega \text {, }
$$

Paso 3)

$$
\begin{aligned}
& \frac{\mathbf{u}^{* *}-\mathbf{u}^{*}}{\Delta t}=-\nabla p^{n+1} \\
& \text { en } \Omega \text {, } \\
& \nabla \cdot \mathbf{u}^{* *}=0 \text { en } \Omega, \quad \mathbf{u}^{* *} \cdot \mathbf{n}=0 \text { sobre } \Gamma, \\
& \frac{\frac{3}{2} \mathbf{u}^{n+1}-\mathbf{u}^{* *}}{\Delta t}=\frac{1}{R e} \nabla^{2} \mathbf{u}^{n+1} \quad \text { en } \Omega \\
& \left.\mathbf{u}^{n+1}\right|_{\mathbf{r}}=\mathbf{g},
\end{aligned}
$$$$
\text { Paso 4) }
$$

en donde el operador B involucra una condición de frontera mixta para la temperatura $\mathrm{y}, \mathbf{u}^{*}$ y $\mathbf{u}^{* *}$ representan velocidades intermedias, de las cuales sólo $\mathbf{u}^{* *}$ satisface la condición de incompresibilidad.

De esta manera, una vez que se obtiene $\theta^{n+1}$ en el primer paso, en el segundo se calcula $\mathbf{u}^{*}$ de manera explícita, pero debido a que $\mathbf{u}^{*}$ no tiene divergencia libre, es decir no satisface la condición de incompresibilidad, el siguiente paso consiste en obtener su proyección sobre el subespacio de divergencia libre, con lo cual obtenemos $\mathbf{u}^{* *}$. Finalmente conocida esta segunda velocidad intermedia puede calcularse $\mathbf{u}^{n+1}$. 
Como puede observarse en el paso 3) para obtener $\mathbf{u}^{* *}$ se requiere conocer el gradiente de la presión, para lo cual $p$ todavía no se conoce. Para solventar esta dificultad tomamos la divergencia a ambos lados de la ecuación del paso 3) y simplificamos, considerando que $\mathbf{u}^{* *}$ satisface la condición de incompresibilidad, así obtenemos la siguiente ecuación de tipo Poisson para la presión

$$
\nabla^{2} p^{n+1}=\frac{1}{\Delta t} \nabla \cdot \mathbf{u}^{*} \quad \text { en } \Omega
$$

Esta ecuación debe suplementarse con una condición de frontera adecuada. Podemos obtener una condición de frontera de tipo Neumann al evaluar la componente normal de la ecuación semidiscreta de momento (3.2b).

$$
\begin{aligned}
& \frac{\partial p^{n+1}}{\partial \mathbf{n}}=\mathbf{n} \cdot\left[\frac{1}{\Delta t}\left(-\frac{3}{2} \mathbf{u}^{n+1}+2 \mathbf{u}^{n}-\frac{1}{2} \mathbf{u}^{n-1}\right)-2\left(\left(\mathbf{u}^{n} \cdot \nabla\right) \mathbf{u}^{\mathbf{n}}\right.\right. \\
& \left.+\frac{1}{R e} \nabla \times\left(\nabla \times \mathbf{u}^{n}\right)\right)+\left(\left(\mathbf{u}^{n-1} \cdot \nabla\right) \mathbf{u}^{\mathbf{n}-1}+\frac{1}{R e} \nabla \times\left(\nabla \times \mathbf{u}^{n-1}\right)\right) \\
& +\frac{R a_{l}}{\left.\operatorname{PrRe^{2}} \theta^{n+1} \mathbf{e}_{2}\right]} \text { sobre } \Gamma
\end{aligned}
$$

en donde el laplaciano de la velocidad se sustituye considerando la relación $\nabla^{2} \mathbf{u}=\nabla(\nabla \cdot \mathbf{u})-\nabla \times \nabla \times \mathbf{u}$. Cabe hacer la observación acerca de que un problema elíptico con condición de frontera Neumann, como el que corresponde a (3.6) y (3.7) para la presión, tiene solución única, salvo una constante arbitraria si se cuenta con una condición de compatibilidad Temam [8], Karniadakis et al. [21].

Para obtener $\theta^{1}$ y $\mathbf{u}^{1}$ requeridos en las ecuaciones de los pasos 1) y 2), así como $p^{1}$, se realiza una subsucesión de los pasos dados en el proceso (3.5) con una aproximación de Euler de primer orden en el primer nivel de tiempo, pero usando un paso de tiempo más pequeño para preservar el segundo orden en precisión de (3.1).

Tomando en cuenta todos estos elementos podemos reordenar el procedimiento en la siguiente forma 


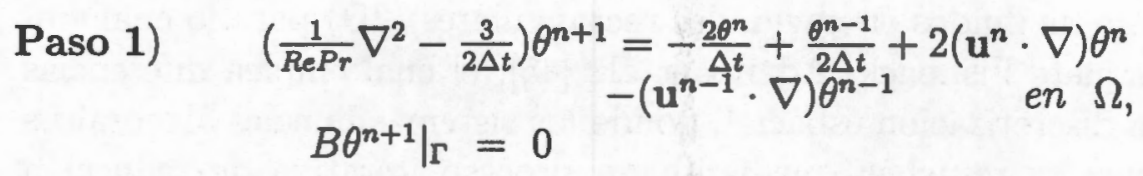

Paso 2) $\quad \begin{aligned} \mathbf{u}^{*}= & \Delta t\left(-2\left(\mathbf{u}^{n} \cdot \nabla\right) \mathbf{u}^{n}+\left(\mathbf{u}^{n-1} \cdot \nabla\right) \mathbf{u}^{n-1}\right. \\ & \left.+\frac{R a_{1}}{\operatorname{PrRe}^{2}} \theta^{n+1} \mathbf{e}_{2}\right)+2 \mathbf{u}^{n}-\frac{1}{2} \mathbf{u}^{n-1} \text { en } \Omega,\end{aligned}$

Paso 3)

$$
\begin{gathered}
\text { subpaso 3.a) } \quad \nabla^{2} p^{n+1}=\frac{1}{\Delta t} \nabla \cdot \mathbf{u}^{*} \quad \text { en } \Omega \\
\begin{array}{c}
\left.\frac{\partial p^{n+1}}{\partial \mathbf{n}}\right|_{\Gamma}=\mathbf{n} \cdot\left[\frac{1}{\Delta t}\left(-\frac{3}{2} \mathbf{u}^{n+1}+2 \mathbf{u}^{n}-\frac{1}{2} \mathbf{u}^{n-1}\right)-2\left(\left(\mathbf{u}^{n} \cdot \nabla\right) \mathbf{u}^{\mathbf{n}}\right.\right. \\
\left.+\frac{1}{R e} \nabla \times\left(\nabla \times \mathbf{u}^{n}\right)\right)+\left(\left(\mathbf{u}^{n-1} \cdot \nabla\right) \mathbf{u}^{\mathbf{n - 1}}+\frac{1}{R e} \nabla \times\left(\nabla \times \mathbf{u}^{n-1}\right)\right) \\
\left.+\frac{R a_{l}}{\operatorname{Pr} R e^{2}} \theta^{n+1} \mathbf{e}_{2}\right]
\end{array}
\end{gathered}
$$

$$
\begin{array}{r}
\text { subpaso 3.b) } \quad \mathbf{u}^{* *}=-\nabla p^{n+1} \Delta t+\mathbf{u}^{*} \quad \text { en } \Omega, \\
\nabla \cdot \mathbf{u}^{* *}=0 \text { en } \Omega, \quad \mathbf{u}^{* *} \cdot \mathbf{n}=0 \text { sobre } \Gamma,
\end{array}
$$

$$
\text { Paso 4) } \quad\left(\nabla^{2}-\frac{3 R e}{2 \Delta t}\right) \mathbf{u}^{n+1}=-\frac{R e}{\Delta t} \mathbf{u}^{* *} \quad \text { en } \Omega,\left.\quad \mathbf{u}^{n+1}\right|_{\Gamma}=\mathbf{g}
$$

De esta manera, puede observarse claramente que las velocidades intermedias $\mathbf{u}^{*}$, en el paso $2, \mathbf{y ~ u}^{* *}$, en el subpaso 3.b), se calculan explícitamente, por lo que la parte costosa del esquema involucra la solución de tres problemas elípticos, uno para la temperatura $\theta^{n+1}$ (con condición de frontera mixta) en el paso 1, otro para la presión $p^{n+1}$ (con condición de frontera Neumann) en el subpaso 3.a), y el último, vectorial, para la velocidad final $\mathbf{u}^{n+1}$ (con condición de frontera Dirichlet) en el paso 4.

Como puede verse, el esquema no requiere iteración alguna, además, es independiente de la dimensión del espacio y de la región donde se presenta el flujo. Para regiones arbitrarias en dos y tres dimensiones puede ser conveniente emplear una discretización espacial por elementos finitos, en tal caso debe proporcionarse una formulación variacional en espacios de funciones de dimensión infinita y posteriormente restringirlo a subespacios de elemento finito de dimensión finita en forma adecuada Glowinski [20], Gunzburger[41].

Por otro lado, para resolver los problemas elípticos que se obtienen del procedimiento numérico, existen resolvedores eficientes independientemente 
del esquema de discretización espacial que se utilice. En este trabajo consideramos el caso de fluidos en cavidades rectangulares (2D) para lo cual empleamos el paquete Fishpack, Adams et al. [45], el cual emplea diferencias finitas para la discretización espacial, donde los sistemas lineales algebraicos que se obtienen se resuelven mediante un proceso iterativo de reducción cíclica eficiente, Sweet [46], y en donde la no unicidad de la ecuación diferencial para la presión, se maneja a través de la solución de mínimos cuadrados ponderados en el sistema algebraico respectivo. Todas las derivadas espaciales de $\theta$, u y $p$ las aproximamos mediante diferencias finitas de segundo orden: centrales, en puntos interiores de la cavidad, o bien regresivas o progresiva, dada por (3.1), en puntos sobre la frontera de la región; de esta manera el esquema completo se basa en aproximaciones de segundo orden.

En el caso de fluidos isotérmicos, se aplica básicamente el mismo procedimiento numérico descrito anteriormente con excepción del paso (1), debido a que no se requiere la ecuación de energía térmica; de esta manera, el proceso inicia en el paso (2) en donde, además, la ecuación de momento (3.2.b) no tiene contribución de la temperatura $\theta$.

\subsection{Resultados numéricos}

Los experimentos numéricos que se muestran, se desarrollan en cavidades rectangulares $\Omega=(0, a) \times(0, b), a, b>0$. Algunos de los resultados reportados corresponden a flujos de estado estacionario, los cuales se obtienen al considerar la convergencia asintótica al estado estacionario, del problema no estacionario, cuanto el tiempo $t$ se aproxima a $+\infty$ (tiempo grande, en la práctica). Para alcanzar la convergencia al estado estacionario asintótico, debe proporcionarse un criterio de convergencia (o de paro), para determinar el tiempo $T_{\text {est }}$; debido a que $T_{\text {est }}$ se considera como el tiempo a partir del cual la solución ya no cambia en cualquier punto del espacio ocupado por el fluido, [12], entonces $T_{\text {est }}$ se determina a partir del criterio discreto de paro absoluto puntual $L_{\infty}$ en la cerradura de la cavidad $\bar{\Omega}$

$$
\left\|f_{h_{x}, h_{y}}^{n+1}-f_{h_{x}, h_{y}}^{n}\right\|_{\infty},
$$

con tolerancia $10^{-5}$, en este caso $f$ se reemplaza por $\mathbf{u} \circ \theta$; y donde $h_{x}$ y $h_{y}$ ( $h$ si $h_{x}=h_{y}$ ) denotan el tamaño de los pasos de la malla en las direcciones $X$ y $Y$ respectivamente. Para flujos térmicos, así como para la mayoría de los isotérmicos se empleó este criterio de convergencia, sin embargo para el 
caso de flujos isotérmicos con $A>1$ fue conveniente recurrir al criterio de paro relativo para determinar $T_{e s t}$. Esto se debe al hecho de que el efecto de la frontera deslizable sobre el flujo se difunde más lentamente al aumentar la razón geométrica $A$, ; como consecuencia de ello la diferencia de la solución entre un paso de tiempo y el siguiente es muy pequeña, con lo cual el criterio absoluto ya no resulta satisfactorio para alcanzar el estado estacionario. En los casos de estudio donde se emplea el criterio de paro relativo, se especifica la tolerancia considerada en cada uno de ellos.

Como es natural, el esquema numérico proporciona resultados en términos de la temperatura, la presión y la velocidad, sin embargo, los que se muestran a continuación se reportan a través de las isotermas (fluidos térmicos) y/o de las líneas de corriente de la función de corriente $\psi$, así como de los isocontornos de la vorticidad $\omega$, a efecto de poder realizar comparaciones con resultados obtenidos por otros autores usando métodos diferentes. Con este objeto, describimos las expresiones matemáticas que relacionan la velocidad del fluido con la vorticidad, y de ésta con la función de corriente

$$
\begin{aligned}
& \bar{\omega}=\nabla \times \mathbf{u} \\
& \nabla^{2} \psi=-\omega
\end{aligned}
$$

Puesto que consideramos el caso bidimensional, la vorticidad es un vector en la dirección $\mathbf{k}$, así $\bar{\omega}=\omega \mathbf{k}$, el cual lo podemos considerar simplemente como el escalar $\omega$; por otro lado, como puede observarse de la segunda relación, debemos resolver un problema elíptico más para obtener la función de corriente $\psi$.

Finalmente, señalamos que presentamos primero experimentos numéricos que corresponden al caso de fluidos térmicos, y posteriormente para fluidos isotérmicos. Los respectivos valores de los parámetros empleados y los valores correspondientes al paso de tiempo $\Delta t$ y al tamaño de la malla $h_{x} \times h_{y}$ se especifican en cada caso de estudio.

\subsubsection{Caso Térmico}

Los experimentos numéricos se realizan en cavidades cuyas fronteras son fijas, en donde la condición de frontera, para la velocidad está dada por $\mathbf{g}=\mathbf{0}$, mientras que para la temperatura se tiene:

$$
\left.\frac{\partial \theta}{\partial n}\right|_{y=0, b}=\left.0 \quad \theta\right|_{x=0}=\left.0.5 \quad \theta\right|_{x=a}=-0.5,
$$


lo que significa que las paredes horizontales están aisladas, la izquierda se encuentra caliente y la derecha fría. Por otro lado, la respectiva condición inicial para la temperatura está dada por $\theta(\mathbf{x}, 0)=0 \mathrm{y}$, considerando que el fluido parte del reposo, se tiene que inicialmente $\mathbf{u}_{o}=(0,0)$. Bajo estas condiciones, los experimentos numéricos se desarrollan para el caso de convección natural, por lo que como se indicó antes $R e=1$ (sección 2.3.1), en cavidades rectangulares, $A=1$ y 3 , que se supone se encuentran llenas de aire, por lo que $\operatorname{Pr}=0.71$, y donde consideramos valores del número de Rayleigh entre $10^{5} \leq R a_{l} \leq 10^{7}$. Los resultados que a continuación se muestran, corresponden al caso de flujos de estado estacionario.

1) Cavidad cuadrada $(a=1=b)$

La Figura 3.1 muestra los resultados obtenidos para $R a_{l}=10^{5}$ usando una malla con $h=\frac{1}{30}$ y un paso de tiempo de $\Delta t=10^{-4}$. Puede observarse de las isotermas que el fluido se calienta en la frontera izquierda lo cual provoca que al tornarse menos denso éste tienda a ascender, debido a la fuerza de flotación, y se extiende por toda la parte superior de la cavidad; al acercarse a la frontera derecha, más fría, el fluido disminuye su temperatura y por lo mismo su densidad aumenta, con lo cual se vuelve más pesado y cae hacia el fondo, en donde se repite el ciclo. Este proceso genera un movimiento de rotación en el sentido de las manecillas del reloj (dirección negativa), lo que queda de manifiesto a través de las líneas de corriente, cuyos valores son negativos. Las isotermas muestran mayor concentración cerca de las paredes con temperatura prescrita, lo que a su vez implica mayor variación de la temperatura en esas zonas; de manera similar las líneas de corriente presentan también mayor cercanía entre ellas hacia las fronteras verticales, manifestando con esto que la velocidad del fluido presenta fluctuaciones importantes en esas regiones; y finalmente, aunque la vorticidad muestra actividad en toda la cavidad, ésta se acentúa hacia la paredes, especialmente en las verticales. Los resultados concuerdan con los obtenidos por Henkes y Hoogendoorn [13], quienes sólo muestran las isotermas y líneas de corriente, a partir del problema estacionario correspondiente y para mallas con $h=\frac{1}{60}$ o más finas y no uniformes.

La gráfica del número de Nusselt local $\left.N u(y)\right|_{x=0}$ sobre la pared caliente, Fig. 3.2, indica que la transferencia de calor es mayor en la parte inferior de ésta y disminuye con la altura. Esto concuerda con el hecho de que las isotermas en la Fig. 3.1 muestran mayor concentración hacia la parte inferior de esta frontera, lo que significa mayor variación de la temperatura, 


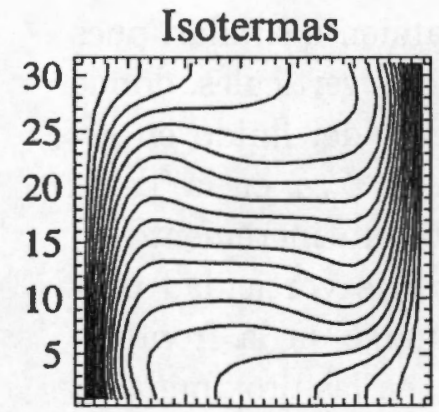

51015202530

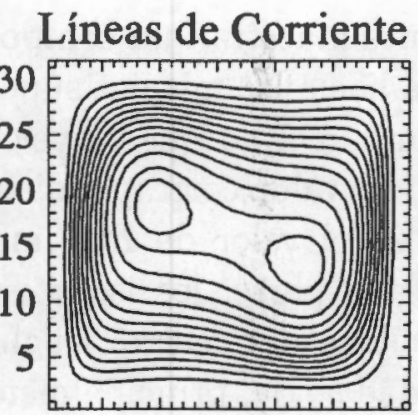

$5 \quad 1015202530$
Isocont. de Vorticidad

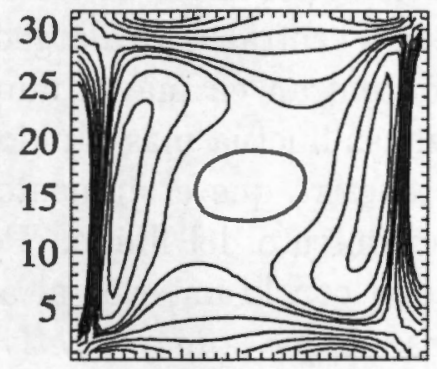

$5 \quad 1015202530$

Fig. 3.1: $R a_{l}=10^{5}, h=\frac{1}{30}$ y $\Delta t=10^{-4}$

horizontalmente, y esta variación disminuye al aumentar la distancia con el fondo de la cavidad, donde las isotermas se alejan entre sí. Los valores correspondientes al número de Nusselt global, $\overline{\mathrm{Nu}}$, el mínimo de la función de corriente, $\psi_{\min }$, así como el tiempo en el que se alcanza el estado estacionario, $T_{\text {est }}$, para este valor de $R a_{l}$, se proporcionan en la Tabla T.1.

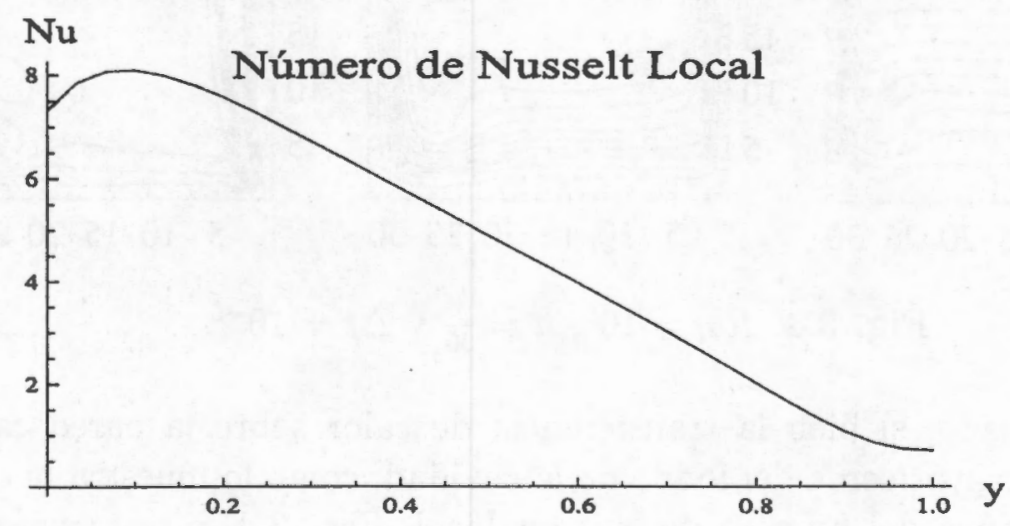

Fig. 3.2: $R a_{l}=10^{5}, A=1, h=\frac{1}{30}$ y $\Delta t=10^{-4}$

Cuando se aumenta el valor de $R a_{l}$ hasta $10^{6}$ y para el mismo valor de $h=\frac{1}{30}$ fue necesario disminuir el paso de tiempo hasta $\Delta t=10^{-5}$. De la Figura 3.3 observamos que, geométricamente, las isotermas muestran un acercamiento hacia las paredes verticales y se aplanan un poco alrededor del centro de la cavidad, lo que implica que horizontalmente la temperatura cambia muy poco en la región central, mientras que las fluctuaciones se manifiestan cerca de las paredes con temperatura fija, en donde se alcanza la mayor 
variación. Las líneas de corriente muestran un comportamiento similar, pues la región central se ve alargada hacia las mismas superficies verticales, donde se concentran en mayor número, por lo que la velocidad del fluido en estas zonas fluctúa más rápido. El valor correspondiente de $\psi_{\min }$ en la Tabla T.1 muestra que el aumento en el valor de $R a_{l}$ implica un incremento en el movimiento del fluido. Por su parte, los isocotornos de vorticidad concuerdan con lo anterior al alejarse del centro y aglutinarse en la frontera, indicando que la mayor actividad se da, principalmente, en las proximidades de las paredes verticales. A pesar de algunas fluctuaciones en las isotermas, hacia las esquinas superior derecha e inferior izquierda, y de que la función de corriente presenta un menor alargamiento de las regiones que apuntan hacia las fronteras horizontales, estos resultados guardan, cualitativamente, buena similitud con los obtenidos en Henkes y Hoogendoorn [13].

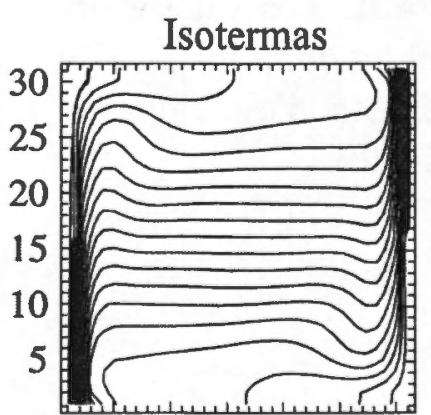

$5 \quad 1015202530$

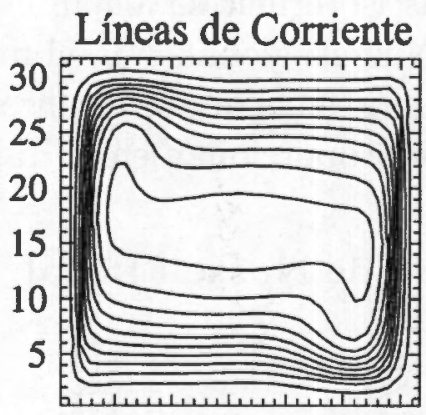

51015202530

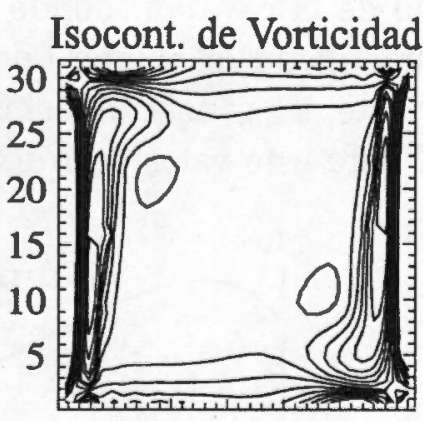

$5 \quad 1015202530$

Fig. 3.3: $R a_{l}=10^{6}, h=\frac{1}{30}$ y $\Delta t=10^{-5}$

Por otro lado, si bien la transferencia de calor sobre la pared caliente sigue siendo mayor cerca del fondo de la cavidad, como lo muestra la correspondiente gráfica del número de Nusselt local, Fig. 3.4, esta transferencia muestra un incremento sustancial, comparado con la Figura 3.2, como habría de esperarse si el gradiente de temperatura aumenta, lo cual también se manifiesta en el valor de $\overline{N u}$ dado en la Tabla T.1; sin embargo, el tiempo $T_{\text {est }}$ que se requiere para llegar al estado estacionario muestra una disminución importante.

Para $R a_{l}=10^{7}$ se requirió disminuir el tamaño de la malla, $h=\frac{1}{80}$, así como el paso de tiempo, $\Delta t=10^{-6}$ y los resultados, Fig. 3.5, muestran una adherencia todavía más notoria hacia las paredes verticales que en los dos casos anteriores de $R a_{l}$ considerados. Tanto las líneas de corriente como los 


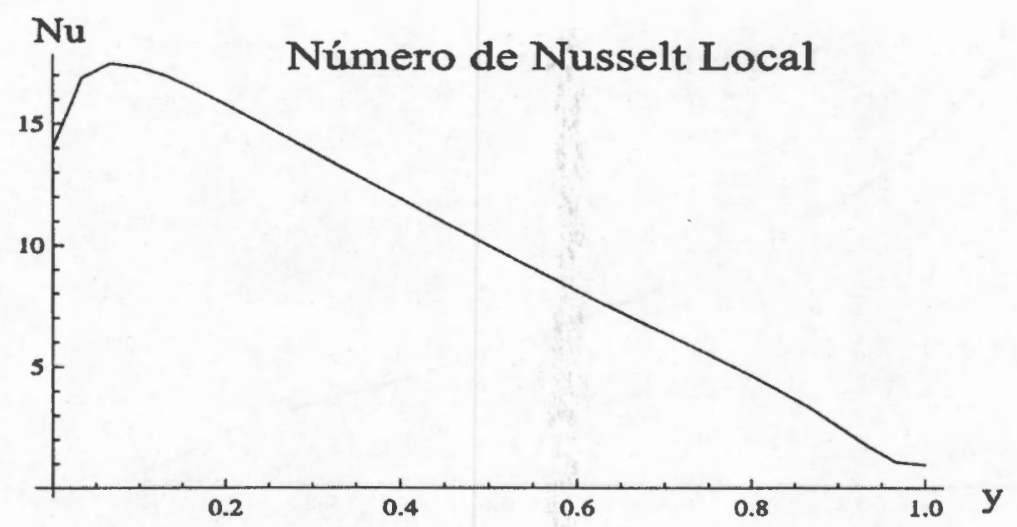

Fig. 3.4: $R a_{l}=10^{6}, A=1, h=\frac{1}{30}$ y $\Delta t=10^{-5}$
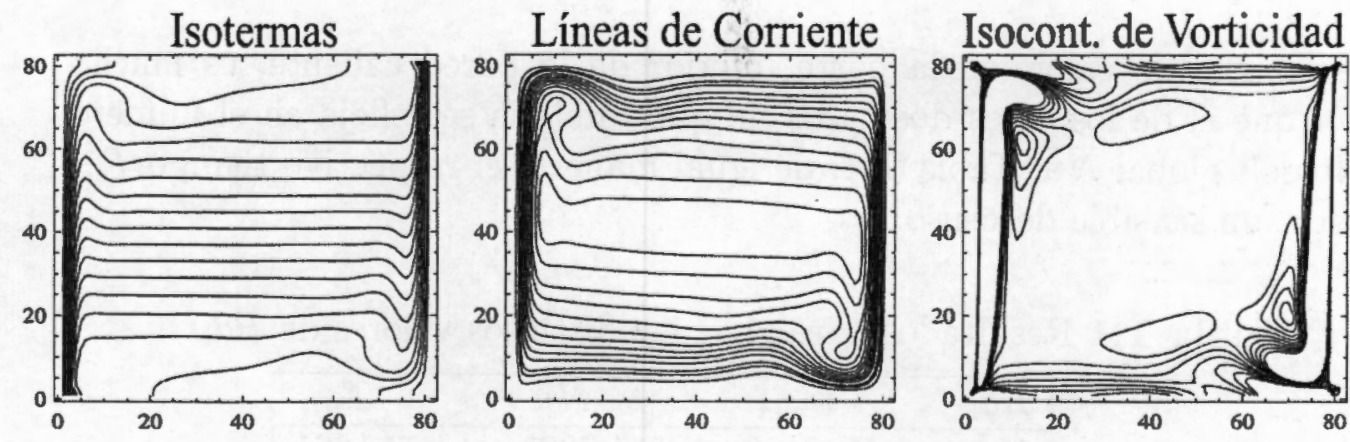

Fig. 3.5: $R a_{l}=10^{7}, h=\frac{1}{80}$ y $\Delta t=10^{-6}$

isocontornos de vorticidad muestran este mismo comportamiento. La región central de las líneas de corriente se ha extendido y puede observarse que algunos de los contornos que apuntan hacia las esquinas superior izquierda e inferior derecha se deforman de tal manera que pareciera que una pequeña porción de uno de ellos va a desprenderse, y aunque esto no sucede aquí, si sugiere la posibilidad de que lo haga para un valor de $R a_{l}$ mayor. Nuevamente, los isocontornos de vorticidad muestran un comportamiento similar, abandonado el centro para concentrarse en la frontera, especialmente en las esquinas superior izquierda e inferior derecha, en donde se exhiben notorias fluctuaciones.

La gráfica del número de Nusselt local para $R a_{l}=10^{7}$ acusa un comportamiento similar a lo observado para los otros valores de $R a_{l}$ pero aún más pronunciado, como lo indica la Figura 3.6, en donde el máximo de la 


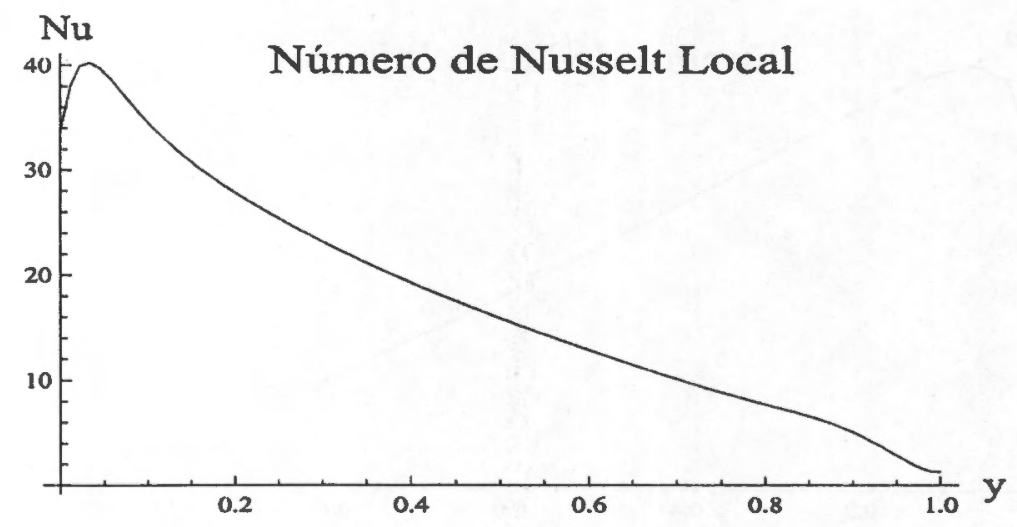

Fig. 3.6: $R a_{l}=10^{7}, A=1, h=\frac{1}{80}$ y $\Delta t=10^{-6}$

transferencia de calor, en la parte inferior de la pared caliente, es mucho mayor que el de los otros dos casos, lo que también se refleja en el número de Nusselt global $\overline{N u}$, Tabla T.1; de igual manera, el respectivo tiempo $T_{\text {est }}$ muestra un sensible descenso.

Tabla T.1 Resultados para $A=1$ y distintos valores de $R a_{l}$

\begin{tabular}{|lrrr|}
\hline$R a_{l}$ & $\psi_{\min }$ & $\overline{N u}$ & $T_{\text {est }}$ \\
\hline $10^{5}$ & -12.8406 & 4.7321 & 0.1661 \\
$10^{6}$ & -19.8707 & 9.8872 & 0.1166 \\
$10^{7}$ & -38.6781 & 17.9693 & 0.0665 \\
\hline
\end{tabular}

2) Cavidad rectangular ( $a=1$ y $b=3$ )

Cuando aumentamos la razón geométrica hasta $A=3$, y para el rango de valores de $10^{5} \leq R a_{l} \leq 10^{7}$, los resultados muestran en general características similares a las obtenidas en el caso de la cavidad cuadrada. $\mathrm{Al}$ aumentar $R a_{l}$ existe, por un lado, mayor tendencia de las isotermas y de las líneas de corriente a aplanarse horizontalmente en el centro de la cavidad, y por otro, estos contornos, junto con los de vorticidad, se encuentran cada vez más cerca de las paredes verticales; además de que, sobre la superficie caliente, las isotermas muestran más cercanía entre sí en la parte inferior de la misma.

La Figura 3.7 muestra el efecto que produce el alargamiento de las paredes isotérmicas para $R a_{l}=10^{5}$. La consecuencia de que el fluido se caliente y se enfríe a lo largo de paredes con mayor longitud, respecto al mismo valor 

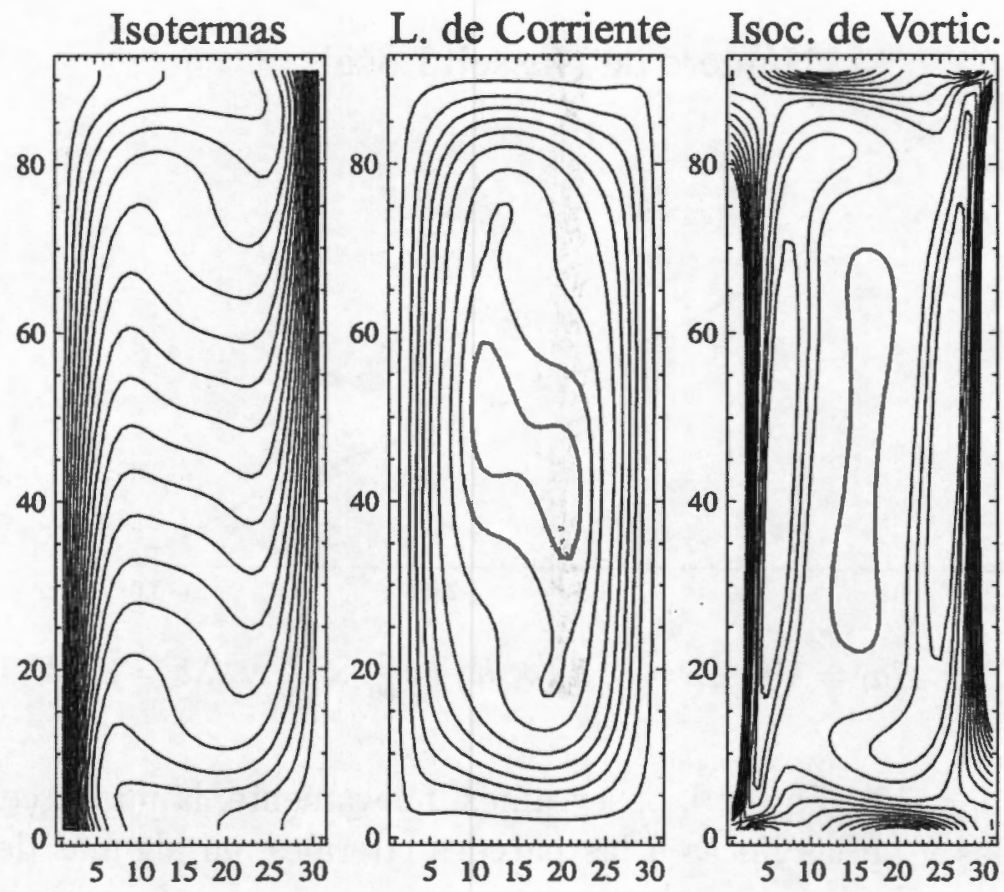

Fig. 3.7: $R a_{l}=10^{5}$ con $h_{x} \times h_{y}=\frac{1}{30} \times \frac{3}{90}$ y $\Delta t=10^{-4}$

de $R a_{l}$ en la cavidad cuadrada, es un importante aumento en el movimiento del fluido, como se puede ver del valor de $\psi_{\min }$ en la Tabla T.2. Esto provoca que las líneas de corriente se expandan y se acerquen a las paredes de la cavidad. Una de estas líneas se ha deformado en algunas partes, de manera similar a lo que se obtiene en la cavidad cuadrada para $R a_{l}=10^{7}$, y apunta en la dirección donde el fluido choca con la pared fría, esquina superior, y con la caliente, esquina inferior. Las isotermas también muestran este efecto y sufren distorsiones en estas zonas donde el fluido choca con las superficies verticales. Los isocontornos de vorticidad llenan prácticamente toda la cavidad, sin embargo, algunos muestran deformaciones, que en el caso de $A=1$ ya aparecen pero de manera más suave, hacia las esquinas mencionadas anteriormente. A pesar de que el fluido es más activo, puede apreciarse de la Figura 3.8 que la transferencia de calor local aumenta ligeramente, respecto del caso de la cavidad cuadrada, aunque la transferencia de calor global, $\overline{N u}$, muestra en cambio un ligero descenso. En cuanto al tiempo $T_{e s t}$, puede apreciarse que el fluido requiere de más tiempo para encontrar el estado estacionario. 


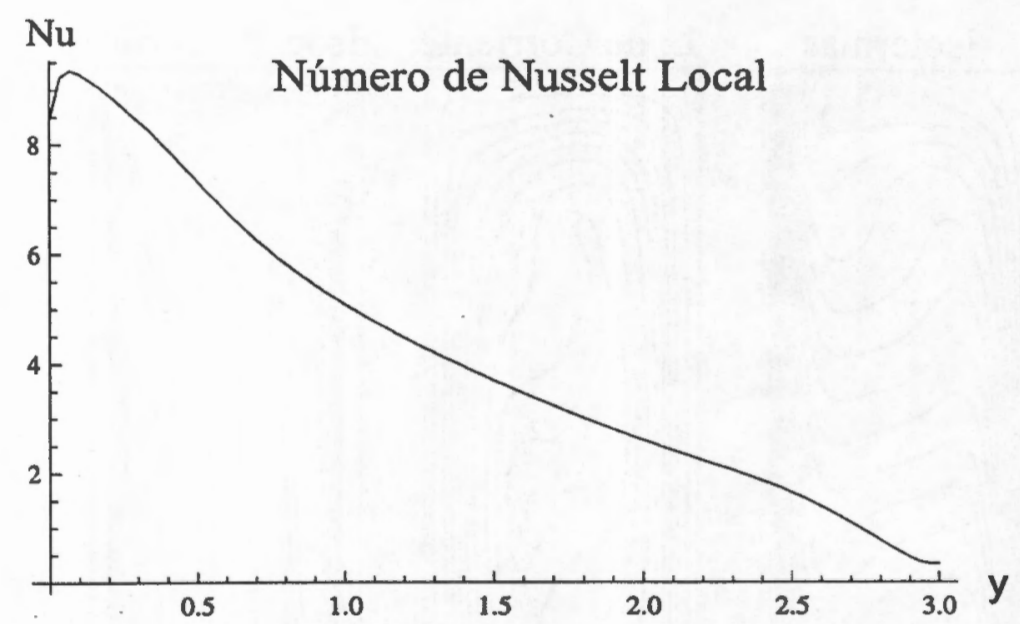

Fig. 3.8: $R a_{l}=10^{5}, A=3, h_{x} \times h_{y}=\frac{1}{30} \times \frac{3}{90}$ y $\Delta t=10^{-4}$

Cuando $R a_{l}=10^{6}$, Fig. 3.9, observamos, nuevamente, la mayor cercanía de las isotermas y de las líneas a las paredes laterales, en algunas de éstas también aparecen deformaciones, aunque más grandes. Los isocontornos de vorticidad están un poco más concentrados hacia los extremos de la cavidad. El valor de $\psi_{\min }$, en la Tabla T.2, señala que el movimiento del fluido se ha incrementado. La correspondiente gráfica del número de Nusselt local, Fig. 3.10 , así como el valor de $\overline{N u}$ muestran que existe un importante aumento en la transferencia de calor, sin embargo, al comparar éstos con el caso respectivo en la cavidad cuadrada, de nuevo se advierte que aunque la transferencia local aumenta un poco, la global disminuye. Algo similar sucede con el tiempo $T_{e s t}$, pues no obstante que el fluido alcanza el estado estacionario más rápido que en el caso anterior, este tiempo es casi el doble del que se requiere para el caso correspondiente en la cavidad cuadrada.

Finalmente, la Figura 3.11 muestra los resultados para $R a_{l}=10^{7}$. Las isotermas y las líneas de corriente muestran todavía mayor tendencia a aplanarse en el centro y a adherirse a las paredes verticales, y las distorsiones que éstas presentan cerca de las paredes laterales disminuyen en tamaño. Puede observarse también que los isocontornos de vorticidad se concentran en mayor número cerca de las paredes laterales, en aquellas regiones donde las distorsiones mencionadas se hacen más notorias, esto es donde el fluido que asciende y el que cae chocan con las superficies horizontales.

La Figura 3.12 muestra que el número de Nusselt local sufre un aumento 

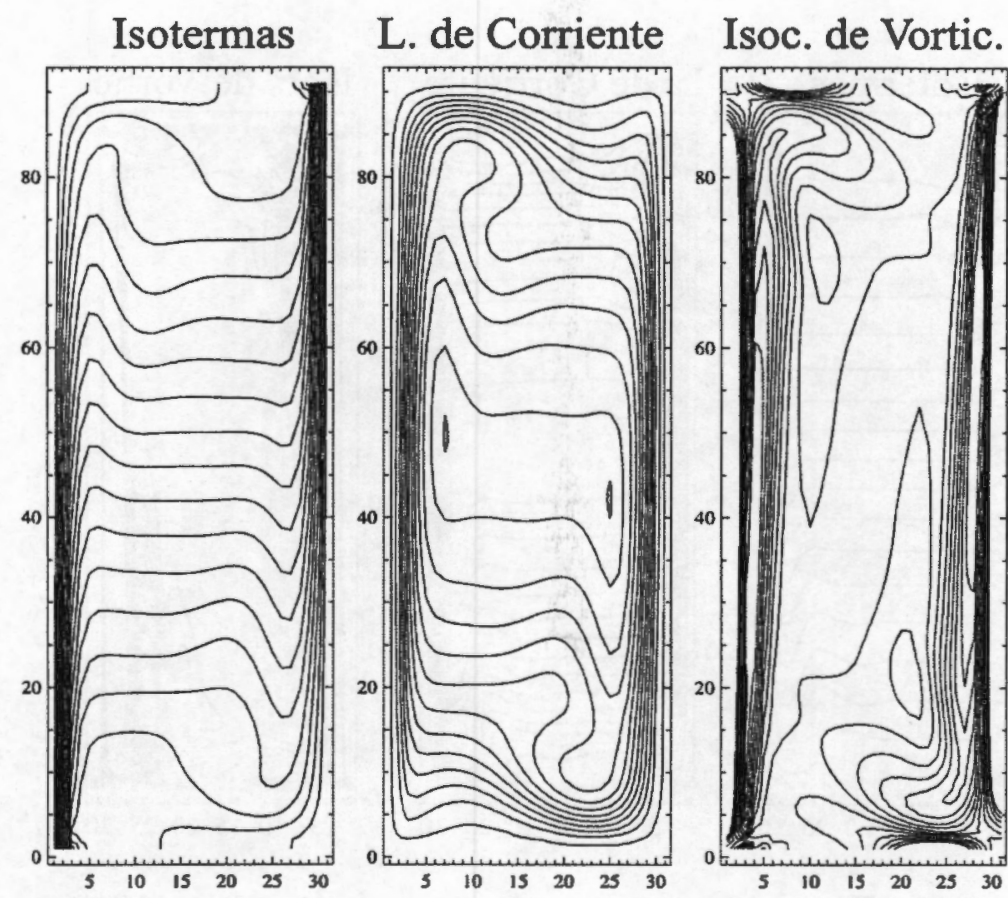

Fig. 3.9: $R a_{l}=10^{6} \operatorname{con} h_{x} \times h_{y}=\frac{1}{30} \times \frac{3}{90}$ y $\Delta t=10^{-5}$

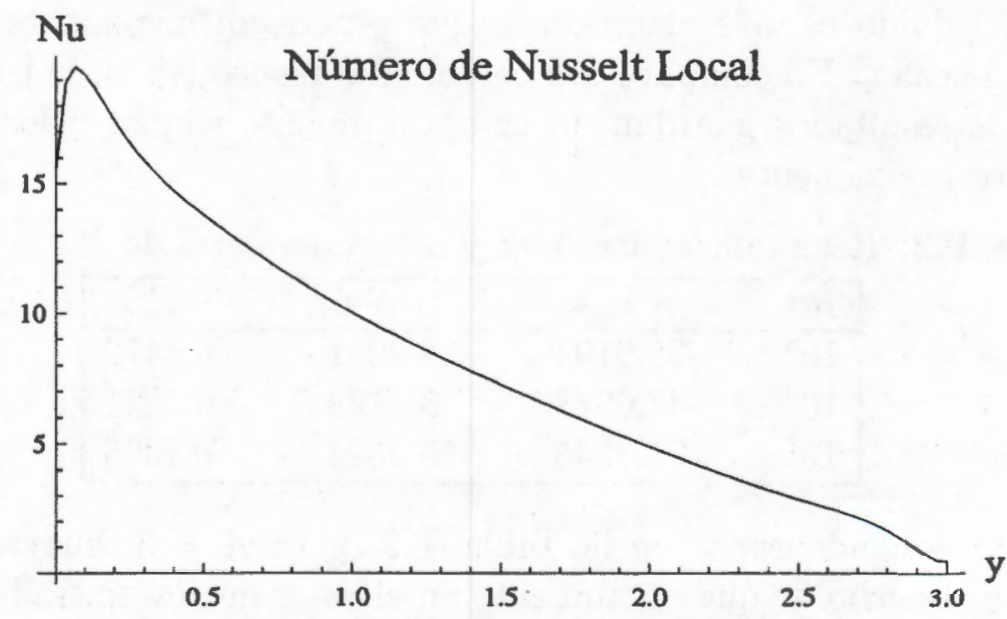

Fig. 3.10: $R a_{l}=10^{6}, A=3, h_{x} \times h_{y}=\frac{1}{30} \times \frac{3}{90}$ y $\Delta t=10^{-5}$ 

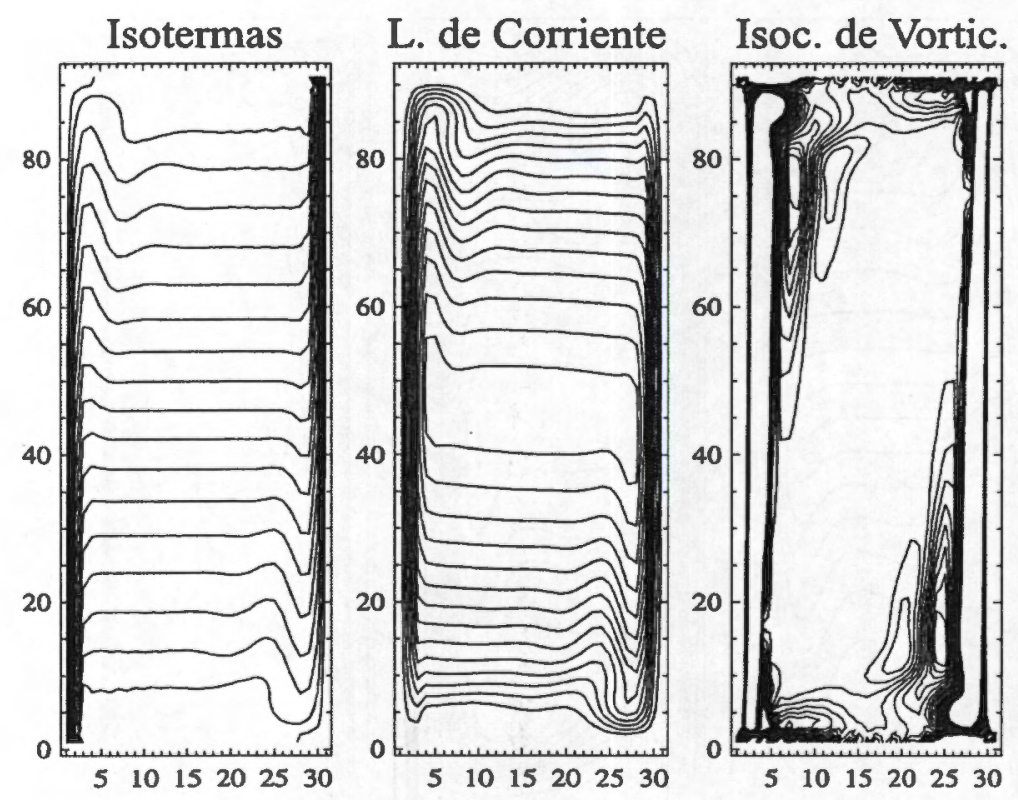

Fig. 3.11: $R a_{l}=10^{7} \operatorname{con} h_{x} \times h_{y}=\frac{1}{30} \times \frac{3}{90}$ y $\Delta t=10^{-6}$

importante, comparado con los dos casos anteriores, especialmente al inicio de la gráfica, lo que concuerda con lo esperado, pues al aumentar $R a_{l}$ el movimiento del fluido es más vigoroso, lo que genera un incremento en la transferencia de calor. En comparación con el caso respectivo en la cavidad cuadrada, estos resultados guardan un comportamiento similar a los otros dos comentados previamente.

Tabla T.2. Resultados para $\mathrm{A}=3$ y distinto valores de $R a_{l}$.

\begin{tabular}{|ccrc|}
\hline$R a_{l}$ & $\psi_{\min }$ & $\overline{N u}$ & $T_{\text {est }}$ \\
\hline $10^{5}$ & -28.9194 & 4.2041 & 0.3472 \\
$10^{6}$ & -47.0948 & 8.1128 & 0.2241 \\
$10^{7}$ & -71.3045 & 15.2521 & 0.1305 \\
\hline
\end{tabular}

Los resultados condensados en la Tabla T.2, para $A=3$, nuevamente hacen evidente el hecho de que un aumento en el valor de $R a_{l}$ se traduce en un movimiento más intenso del fluido, como lo indica la magnitud de $\psi_{\min }$. Esta situación genera a su vez una mayor transmisión de calor, cuantificada globalmente por $\overline{N u}$. En cambio, el tiempo $T_{\text {est }}$ requerido para alcanzar el 


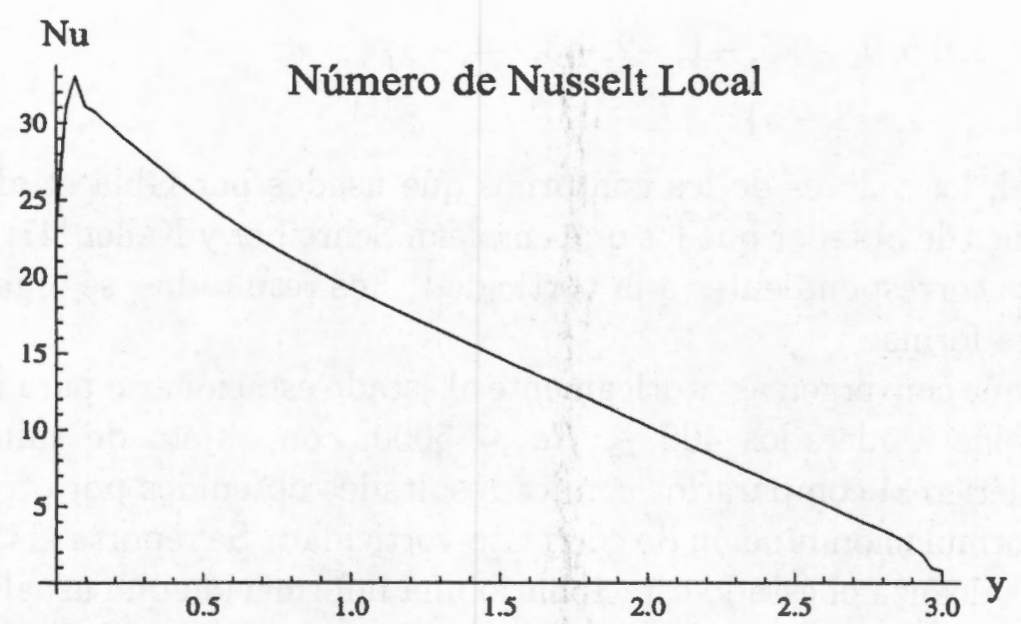

Fig. 3.12: $R a_{l}=10^{7}, A=3, h_{x} \times h_{y}=\frac{1}{30} \times \frac{3}{90}$ y $\Delta t=10^{-6}$

estado estacionario disminuye.

\subsubsection{Caso Isotérmico}

Los resultados se obtienen considerando que la cavidad tiene tapa superior deslizable, así, la condición de frontera para la velocidad $\mathbf{u}$ está dada por $\mathbf{g}=(1,0)$ sobre la pared móvil $(x, y=b)$, mientras que $\mathbf{g}=(0,0)$ en el resto de la frontera. Puesto que se considera que el fluido parte del reposo, la condición inicial para la velocidad es $\mathbf{u}_{o}=(0,0)$.

Los experimentos numéricos que se presentan corresponden a valores moderados y altos del número de Reynolds en el rango de $400 \leq R e \leq 15000$. Los valores para las líneas de corriente y para los contornos de vorticidad, son los mismos que los de Ghia et al. [48], con excepción del caso para $R e=4000$ donde empleamos los valores considerados por Schreiber and Keller, [47]. Los valores para las líneas de corriente considerados por Ghia et al., cg, y por Schreiber y Keller, cs, respectivamente son:

$$
\begin{aligned}
& \mathrm{cg}=\left\{3 \times 10^{-3}, 1.5 \times 10^{-3}, 10^{-3}, 5 \times 10^{-4}, 2.5 \times 10^{-4}, 10^{-4}, 5 \times 10^{-5},\right. \\
& 10^{-5}, 10^{-6}, 10^{-7}, 10^{-8},-10^{-10},-10^{-7},-10^{-5},-10^{-4},-0.01 \text {, } \\
& -0.03,-0.05,-0.07,-0.09,-0.1,-0.11,-0.115,-0.1175\} \text {. } \\
& \mathrm{cs}=\{-0.01,-0.02,-0.04,-0.06,-0.08,-0.1,-0.11\}
\end{aligned}
$$

De manera análoga, los valores para los isocontornos de vorticidad usados por Ghia et al., vg, y por Schreiber y Keller, vs, son: 


$$
\begin{aligned}
& \mathbf{v g}=\{3,2,1,0.5,0,-0.5,-1,-2,-3,-4,-5\} \\
& \mathbf{v s}=\{5,3,1,-1,-3,-5\}
\end{aligned}
$$

En general, los valores de los contornos que usados por Ghia et al. [48] son más difíciles de obtener que los que emplean Schreiber y Keller [47], principalmente los correspondientes a la vorticidad. Los resultados, se organizan en la siguiente forma:

1) Flujos que convergen asintóticamente al estado estacionario para números de Reynolds moderados $400 \leq R e \leq 5000$, con objeto de validar el esquema numérico al compararlos con los resultados obtenidos por otros autores con la formulación función de corriente-vorticidad. Se reporta el tiempo $T_{\text {est }}$ en que se alcanza el estado estacionario una información que usualmente no se considera cuando se resuelve el problema no estacionario. 2) Para $R e=4000$ se muestra la evolución del flujo a distintos tiempos antes de $T_{e s t}$. 3) Para valores grandes del número de Reynolds se reportan flujos dependientes del tiempo; para $R e=10000$ a distintos tiempos y para $R e=15000$ en dos tiempos, uno cerca del inicial y otro a un tiempo mayor; en estas tres primeras secciones, los experimentos tienen lugar en la cavidad cuadrada unitaria. 4) Se reportan flujos de estado estacionario en cavidades verticales (razón geométrica $A>1$ ) para $R e=1000$.

1) Flujos que convergen asintóticamente al estado estacionario:

En la Figura 3.13 se muestra el flujo que se obtiene para $R e=1000$, con $h=\frac{1}{80}$ y $\Delta t=0.01$, en $T_{\text {est }}=31.15$. Como puede observarse de las líneas de corriente, aparecen tres vórtices bien formados, uno primario que gira en dirección negativa y dos secundarios que lo hacen en la dirección positiva, mientras que la vorticidad se manifiesta de manera más intensa cerca de la frontera. Este resultado concuerda bien con el que se muestra en Ghia et al. [48], donde $h=\frac{1}{128}$, así como con las líneas de corriente que presentan Erturk et al., [49], quienes emplean una malla uniforme con $h=\frac{1}{600}$ (en este último trabajo no se reportan los isocontornos de la vorticidad).

El flujo de la Figura 3.14 corresponde al caso $R e=4000$, con $h=\frac{1}{128}$ y $\Delta t=0.005$, en un tiempo final $T=37.5$. Este tiempo corresponde al momento en que el flujo concuerda con el reportado en Schreiber y Keller [47] para el problema estacionario y en donde $h=\frac{1}{160}$; y no se reporta en Ghia et al. [48] ni en Erturk et al. [49]. El tiempo en que se alcanza el estado estacionario es $T_{\text {est }}=86.88$, y el flujo correspondiente a este tiempo muestra sólo pequeñas diferencias respecto al mostrado en la Figura 3.14. 

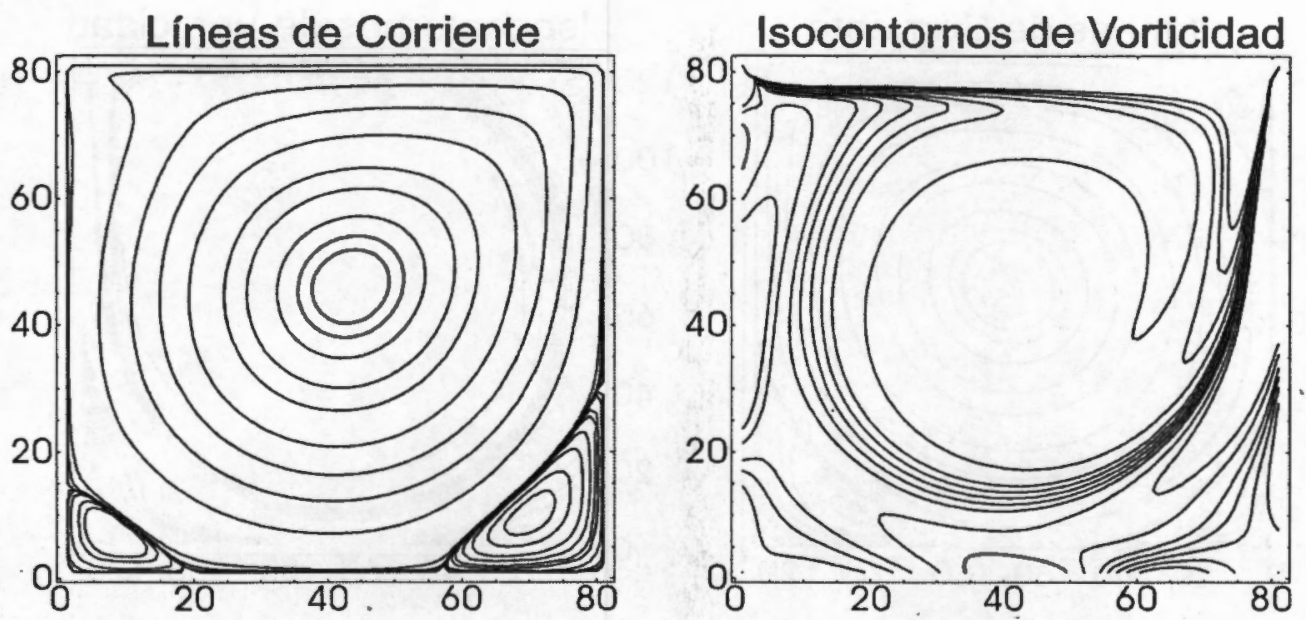

Fig. 3.13: $R e=1000, h=\frac{1}{80}, \Delta t=0.01, T_{\text {est }}=31.15$

Nuevamente, en las líneas de corriente puede apreciarse un vórtice principal, sin embargo, el número de pequeños subvórtices es mayor, por uno, que el obtenido para $R e=1000$; por su parte los isocontornos de vorticidad indican que ésta se manifiesta principalmente hacia las paredes de la cavidad.
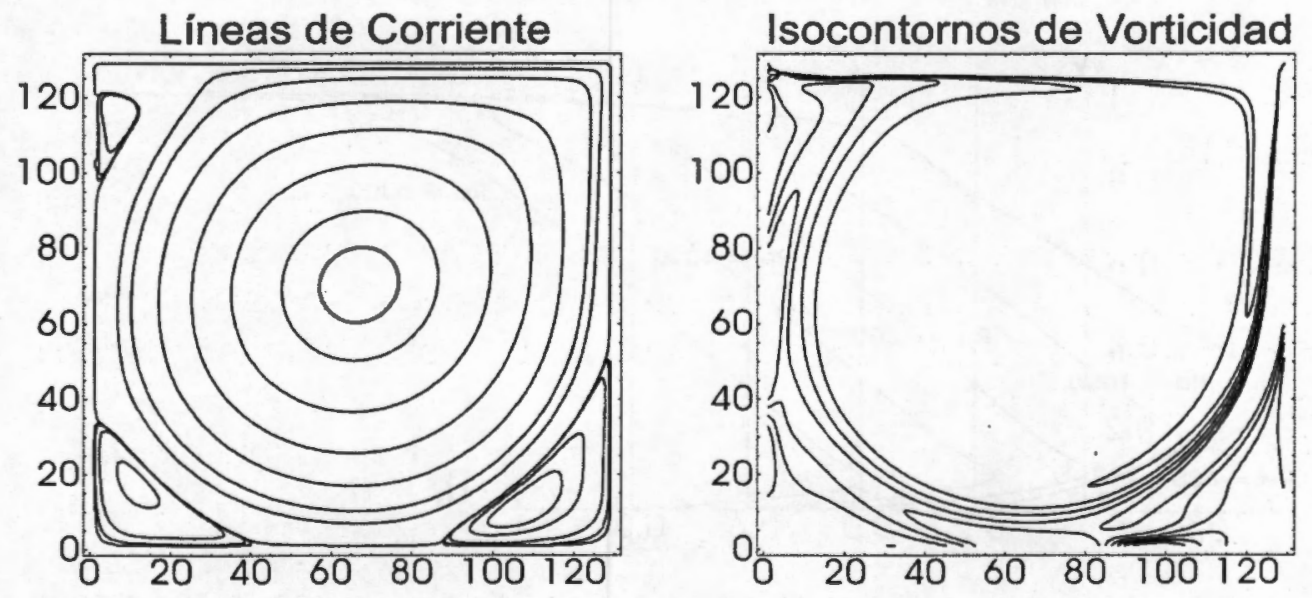

Fig. 3.14: $R e=4000, h=\frac{1}{128}, \Delta t=0.005, T=37.5$

En el flujo que se obtiene para $R e=5000$ en $T_{\text {est }}=121.884, \operatorname{con} h=\frac{1}{128} \mathrm{y}$ $\Delta t=0.004$, Figura 3.15, se observa la misma cantidad de vórtices secundar rios que en el caso anterior, pero un poco más grandes, además un pequeño vórtice terciario, girando en dirección negativa, hace su aparición en la es- 

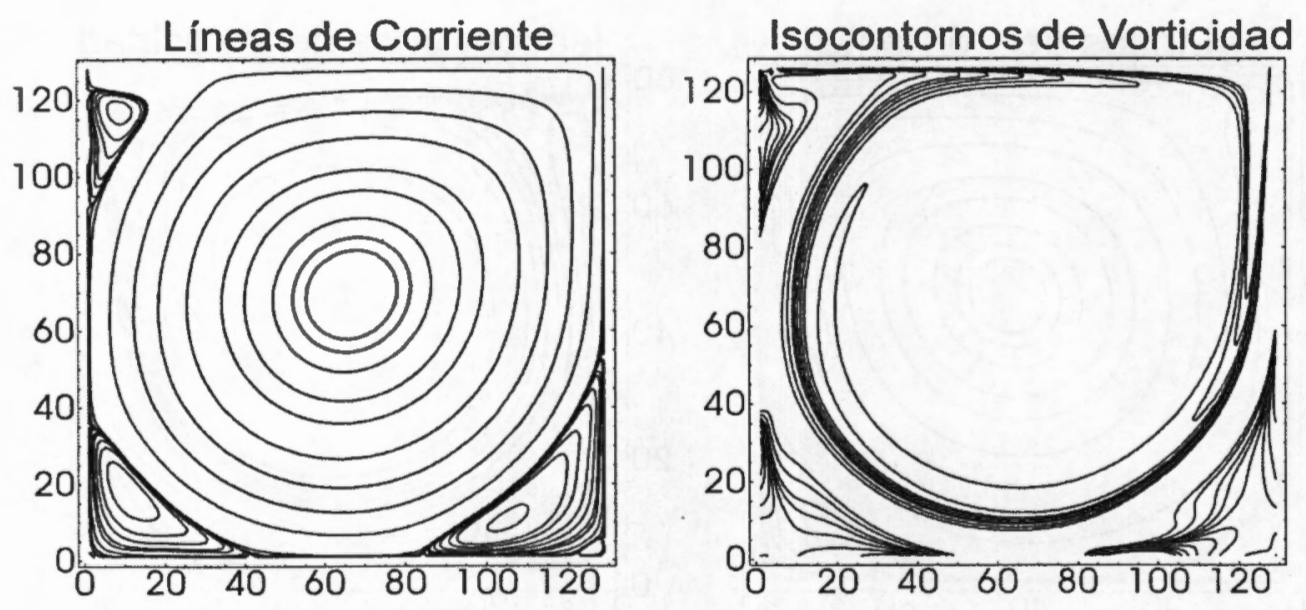

Fig. 3.15: $R e=5000, h=\frac{1}{128}, \Delta t=0.004, T_{\text {est }}=121.884$

quina inferior izquierda. Los contornos de vorticidad, más desarrollados, se mantienen nuevamente cerca de la frontera, indicando mayor actividad en dicha zona. El resultado concuerda con el que se obtiene en Ghia et al. [48], con $h=\frac{1}{256}$, así como con las líneas de corriente obtenidas en Erturk et al. [49] usando $h=\frac{1}{600}$.

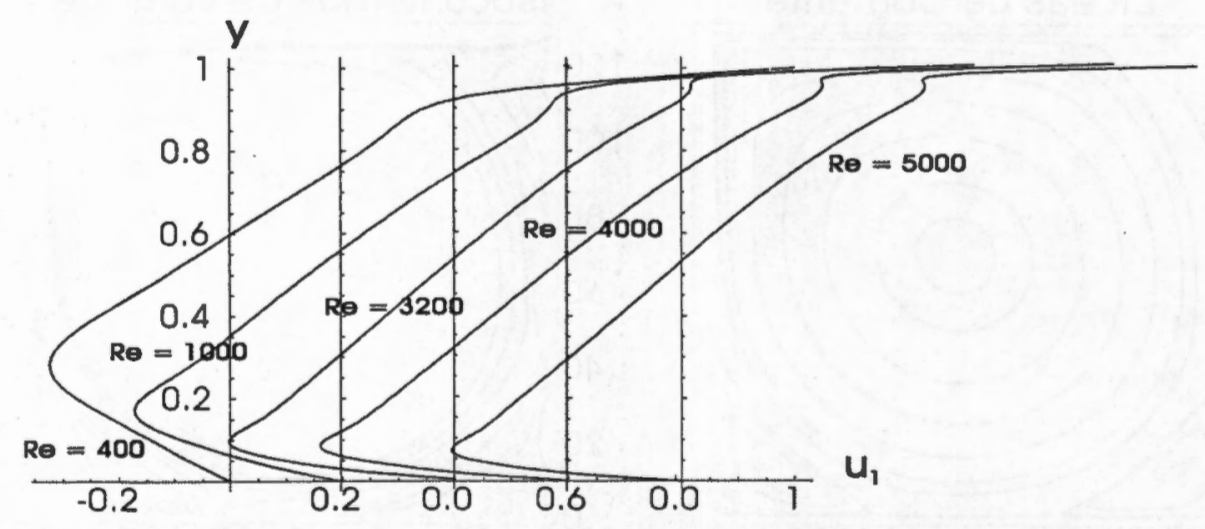

Fig. 3.16: Perfiles de velocidad horizontal en $x=0.5$ para $400 \leq R e \leq 5000$

A partir de los pasos de tiempo, $\Delta t=0.004$ y $\Delta t=0.005$, empleados para $R e=5000$ y $R e=4000$ respectivamente, con el mismo $h$, se deduce que $\Delta t$ debe disminuir cuando $R e$ aumenta para capturar el incremento en la dinámica del flujo.

Se obtuvieron también los flujos correspondientes para $R e=400$, en 


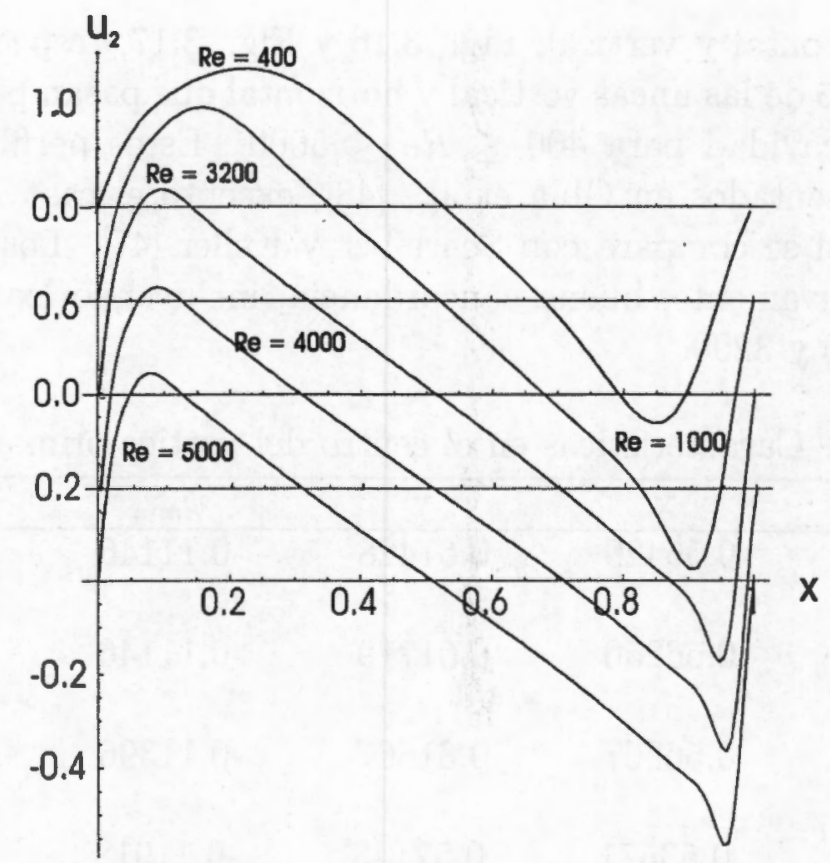

Fig. 3.17: Perfiles de la velocidad vertical en $y=0.5$ para $400 \leq R e \leq 5000$

$T_{\text {est }}=23.08$ con $h=\frac{1}{60}$ y $\Delta t=0.01$, así como para $R e=3200$, en $T_{\text {est }}=67$ con $h=\frac{1}{128}$ y $\Delta t=0.005$; ambos casos concuerdan con los que se reportan en Ghia et al. [48]. Para este rango de valores de $R e$, $400 \leq R e \leq 5000$, el tamaño de malla empleado en este trabajo es significativamente más grande que los que usan aquellos autores con cuyos trabajos estamos comparando. Además, en todos los casos, existe buena concordancia con los valores obtenidos de $T_{\text {est }}$, los cuales aumentan a medida que $R e$ se incrementa, esto es, el fluido requiere de más tiempo para alcanzar el estado estacionario a medida que el movimiento del mismo es mayor. Por otro lado, aunque los tiempos $T=37.5$ y $T_{\text {est }}=86.88$ para $R e=4000$ pueden parecer extraños, la coincidencia de los flujos obtenidos, en esta sección, en los tiempos $T_{\text {est }}$ indicados, con los flujos de estado estacionario presentados en [48] para $R e=400,1000,3200$ y 5000, junto con el hecho de que es más fácil satisfacer los valores de los contornos dados en Schreiber y Keller [47] que aquellos empleados en Ghia et al. [48], implican que nuestra manera de determinar $T_{\text {est }}$ conduce a que los respectivos valores de contorno que obtenemos, presentan mayor coincidencia con los que se muestran en [48].

Para reforzar la validación del método numérico, se muestran los perfiles 
de velocidad, horizontal y vertical, Fig. 3.16 y Fig. 3.17, respectivamente, obtenidos a lo largo de las líneas vertical y horizontal que pasan por el centro geométrico de la cavidad para $400 \leq R e \leq 5000$. Estos perfiles se comparan con los presentados en Ghia et al. [48], excepto el caso $R e=4000$ a $T=37.5$, el cual se compara con Schreiber y Keller [47]. Los resultados muestran, cualitativamente, buena concordancia, incluyendo los correspondientes a $R e=400$ y 3200 .

Tabla I.1. Características en el centro del vortice primario.

\begin{tabular}{|cccccc|}
\hline $\mathrm{Re}$ & $\mathrm{h}$ & $\mathrm{x}$ & $\mathrm{y}$ & $\psi_{\min }$ & $\omega$ \\
\hline 400 & $\frac{1}{140}$ & 0.56429 & 0.61428 & -0.11146 & -2.25923 \\
400 & $\frac{1}{128}$ & 0.56250 & 0.61719 & -0.11146 & -2.27267 \\
400 & $\frac{1}{60}$ & 0.56667 & 0.61667 & -0.11396 & -2.33054 \\
1000 & $\frac{1}{140}$ & 0.53571 & 0.57143 & -0.11918 & -2.12054 \\
1000 & $\frac{1}{128}$ & 0.53906 & 0.57031 & -0.11948 & -2.12790 \\
1000 & $\frac{1}{80}$ & 0.53750 & 0.57500 & -0.12025 & -2.14618 \\
3200 & $\frac{1}{128}$ & 0.52344 & 0.54688 & -0.12233 & -1.96368 \\
4000 & $\frac{1}{160}$ & 0.51875 & 0.53750 & -0.12170 & -1.99651 \\
4000 & $\frac{1}{128}$ & 0.52344 & 0.53906 & -0.11368 & -1.93892 \\
5000 & $\frac{1}{256}$ & 0.51953 & 0.54297 & -0.11992 & -1.76250 \\
5000 & $\frac{1}{128}$ & 0.52344 & 0.53906 & -0.12118 & -1.86258 \\
\hline
\end{tabular}

La ubicación del centro del vórtice primario así como los valores de la función de corriente, que corresponde a un mínimo, y de la vorticidad en este centro, se muestran en la Tabla I.1. Se realizaron comparaciones con Schreiber y Keller [47] y Ghia et al. [48], empleando las mismas mallas usadas en estos trabajos, así como algunas otras, y los diferencias observadas con [48], fueron menos de $3 \%$ para la función de corriente y menos de $6 \%$ para 
la vorticidad. (Para $R e=4000$ las diferencias mostradas, al comparar con Schreiber y Keller [47], fueron de $8.30 \%$ para la función de corriente y $10.61 \%$ para la vorticidad). Para $R e=1000$, al comparar los valores obtenidos por Erturk et al. [49], en donde usan $h=\frac{1}{600}$, con los correspondientes valores obtenidos aquí con $h=\frac{1}{140}, \frac{1}{128}, \mathrm{y} \frac{1}{80}$ las diferencias son, menos de $1.3 \%$ para la función de corriente $(0.34 \%, 0.59 \%$ y $1.24 \%$ respectivamente) y menos de $4 \%$ para la vorticidad $(2.67 \%, 3.02 \%, 3.91 \%)$; mientras que para $R e=5000$, con $h=\frac{1}{256}$ y $\frac{1}{128}$ las discrepancias son, menos de $2 \%$ para la función de corriente $(1.14 \%, 0.09 \%)$ y a lo más $9.3 \%$ para la vorticidad $(9.3 \%, 3.44 \%)$.

2) Flujo transitorio, $\operatorname{Re}=4000$ :

Se reportan resultados para $R e=4000$ con $h=\frac{1}{128}$ y $\Delta t=0.005$ que muestran la evolución del flujo en diferentes tiempos anteriores a $T=37.5$, Fig. 3.14, el cual corresponde al tiempo en que el flujo concuerda con el que obtienen, a partir del problema estacionario, Schreiber y Keller [47]; para efectos de comparar el flujo, obtenido en este caso, consideramos los mismos
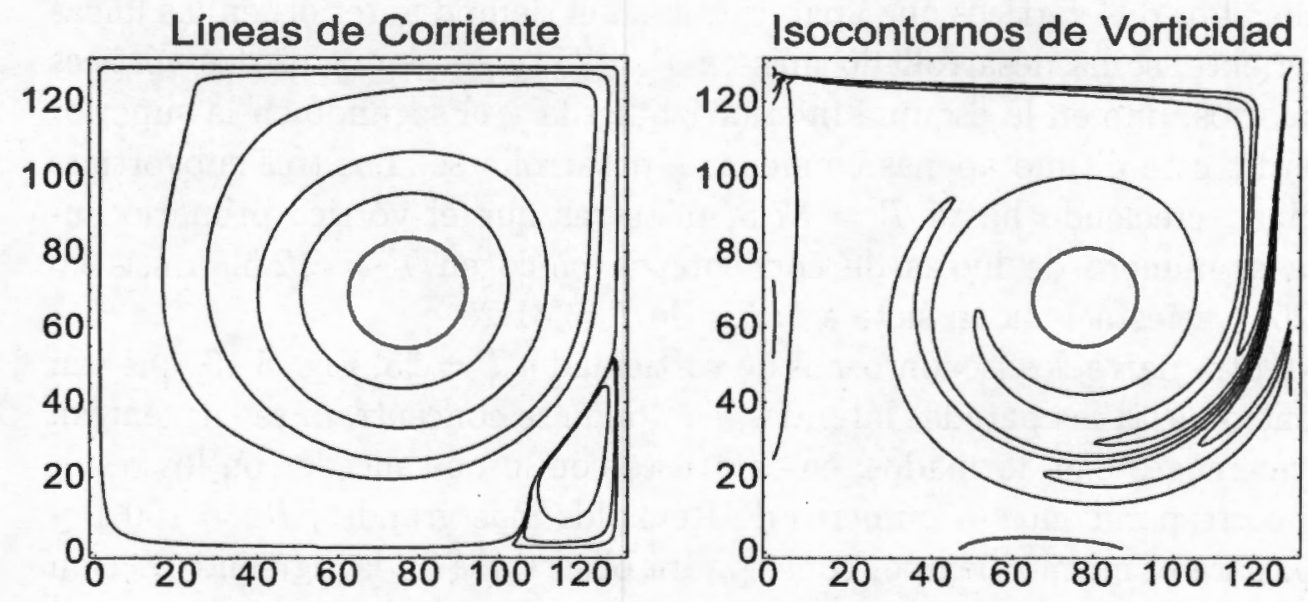

Fig. 3.18: $R e=4000, h=\frac{1}{128}, \Delta t=0.005, T=12.5$

valores de los contornos empleados en ésta referencia. Los flujos obtenidos en los tiempos $T=12.5, T=25$, y $T=31.25$ se muestran sucesivamente, Fig. 3.18, Fig. 3.19 y Fig. 3.20 , y puede observarse con claridad las características de la evolución hasta $T=37.5$.

Al tiempo $T=12.5$ apenas inicia el desarrollo del flujo, que proviene de la frontera derecha, lo cual se implica por la aparición del primer vórtice 

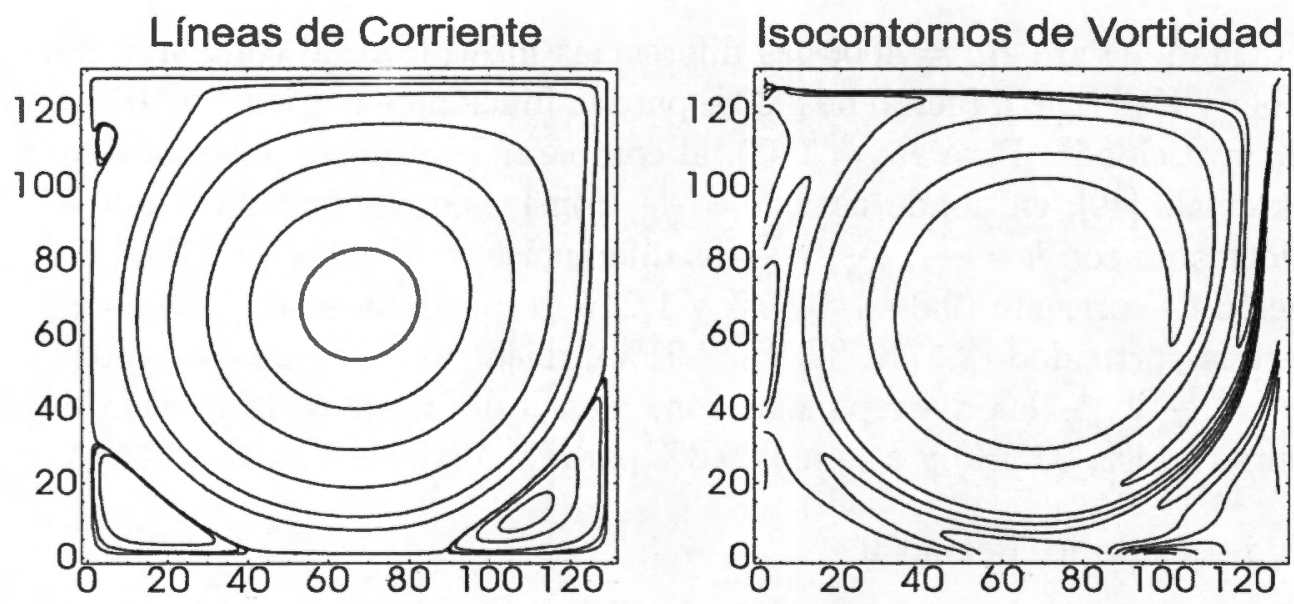

Fig. 3.19: $R e=4000, h=\frac{1}{128}, \Delta t=0.005, T=25$

secundario en la esquina inferior derecha en las líneas de corriente, así como por el hecho de que los isocontornos de vorticidad se concentran en la pared derecha. Los dos vórtices que aparecieron en el tiempo anterior, en las líneas de corriente, se ha desarrollado más en $T=25$, y surgen otros dos vórtices secundarios, uno en la esquina inferior izquierda y el segundo en la superior izquierda, este último apenas comienza a desarrollarse. Los tres subvórtices continuan creciendo hasta $T=37.5$, mientras que el vórtice primario aumenta el número de líneas de corriente de cinco en $T=12.5$, a seis en $T=25$, y se estaciona en siete a partir de $T=31.25$.

Por otra parte, los isocontornos de vorticidad a $T=25$, Fig. 3.19, que van del centro hasta las paredes laterales, en donde se concentran, se encuentran uniformemente bien formados, en contraste con lo que sucede con los resultados correspondientes a números de Reynolds más grandes, $R e=10000$ y 15000, para el mismo tiempo, como podrá observarse en la siguiente sección de resultados.

3) Flujos dependientes del tiempo, $R e=10000$ y $R e=15000$ :

Cuando $R e=10000$ se observa que no se alcanza un estado estacionario. Esto coincide con la conclusión a la que han llegado varios autores: el flujo es dependiente del tiempo tan pronto como $R e>7500$, esto es, aparece la transición a flujos oscilatorios, lo cual sólo puede observarse cuando se realizan cálculos para tiempos muy grandes; con este objetivo, se muestran resultados hasta un tiempo de $T=5000$, el cual representa un tiempo con- 

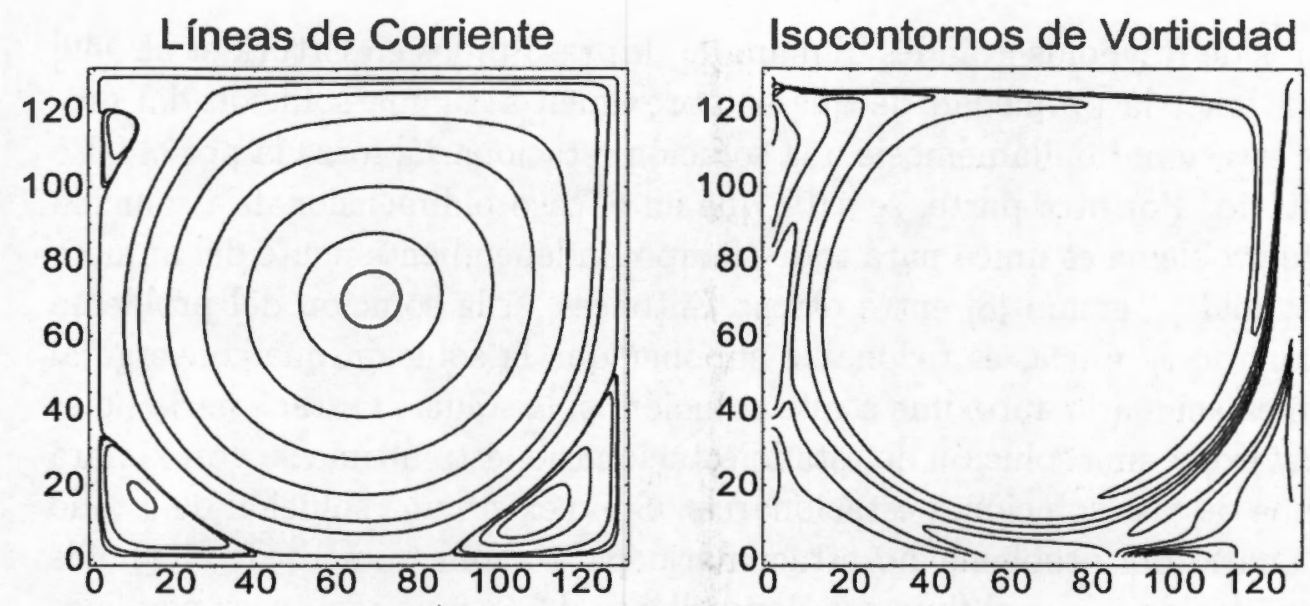

Fig. 3.20: $R e=4000, h=\frac{1}{128}, \Delta t=0.005, T=31.25$

siderablemente grande comparado con aquellos $T_{\text {est }}$ en que los flujos de la subsección 1) alcanzan el estado estacionario.

A continuación se presentan una serie de resultados para $R e=10000$ con $h=\frac{1}{128}$ y $\Delta t=0.004$ con el objeto de seguir la evolución del flujo a diferentes tiempos, comenzando a partir de la condición inicial de reposo. Cabe hacer notar que: i) el paso de tiempo $\Delta t$ empleado es el mismo que el que se utilizó para $R e=5000$, lo cual significa que la estabilidad del esquema numérico no es sensible a este aumento del número de Reynolds. ii) El tamaño de la malla $h=\frac{1}{128}$ es dos veces más gruesa que aquella empleada en Ghia et al. [48] y Sahin y Owens [50], y mucho más gruesa comparada con $h=\frac{1}{600}$ que usan en Erturk et al. [49]. iii) Los resultados a $T=208$, Fig. 3.24, concuerda bien con los obtenidos en [48], y con las líneas de corriente de [49], y parcialmente con los correspondientes resultados en [50], ya que estos últimos obtienen seis líneas de corriente en el vórtice primario de la función de corriente mientras que en [48] y [49], así como en nuestros resultados se obtienen nueve. iv) Respecto a los resultados en [48] y [49] debemos señalar que debido a que los números de Reynolds considerados en la subsección 1) no son grandes, la solución del problema no estacionario para $t$ suficientemente grande aproxima el flujo de estado estacionario, que por otra parte debe aproximarse a la solución única del correspondiente problema estacionario. En la referencia [49], a través de una técnica de falso transitorio, el problema estacionario se resuelve para números de Reynolds grandes, hasta $R e=21000$. Debido a que se sabe que la solución del problema estacionario no es única para 
números de Reynolds grandes Temam [8], los resultados en Erturk et al. [49] pueden tener la propiedad de que se aproximen a alguna solución del problema estacionario, llamémosle una solución estacionaria, forzada por el falso transitorio. Por otra parte, se sabe que en el caso bidimensional la solución de este problema es única para todo tiempo, independientemente del número de Reynolds, Temam [8] entre otros. Entonces, si la solución del problema estacionario es única, es razonable suponer que la solución que converge al estado estacionario aproxime a esta solución; si la solución estacionaria no es única y existe una solución de estado estacionario, ésta última se aproximará a alguna de esas soluciones estacionarias. Si no existe una solución de estado estacionario del problema no estacionario para algún número de Reynolds grande, debido a que el flujo sea dependiente del tiempo, entonces podemos hacernos la siguiente pregunta ¿el flujo pasa por una solución del problema estacionario, a cierto tiempo? En Ghia et al. [48] se resuelve el problema no estacionario haciendo uso de las variables función de corriente y vorticidad; en este trabajo, no queda claro si la solución que obtienen corresponde al flujo en algún tiempo específico o si es la de estado estacionario. En cualquier caso, su concordancia con el flujo obtenido por Erturk et al. [49] significa que tal solución aproxima la solución del problema estacionario en cierto tiempo; entonces, nuestra solución en $T=208$ coincide, numéricamente hablando, con la de ellos, y continúa su evolución. Los resultados se comentan a continuación.
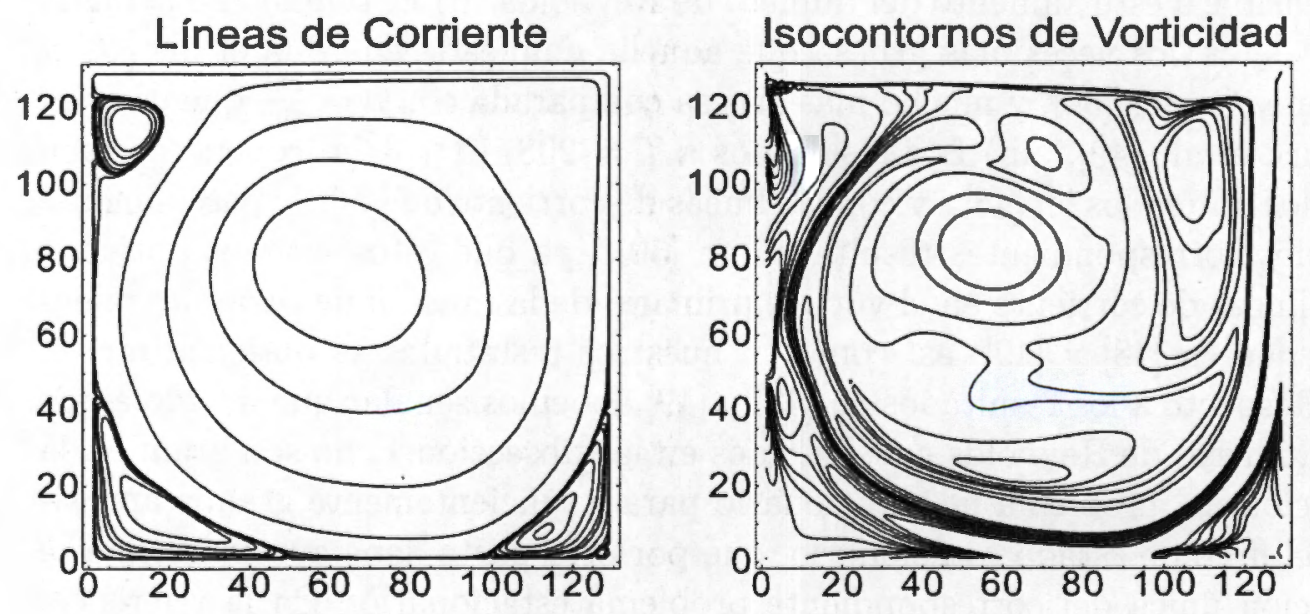

Fig. 3.21: Fig. 9. $R e=10000, h=\frac{1}{128}, \Delta t=0.004, T=25$

El flujo a $T=25$, Fig. 3.21, muestra una forma irregular en los contornos 
de las líneas de corriente y de la vorticidad, comparados con el flujo para $R e=4000$ obtenidos en el mismo tiempo, Fig. 3.19 de la subsección anterior, en esta última la vorticidad está abandonando el centro mientras que en la Figura 3.21 se extiende por toda la cavidad. El pequeño vórtice terciario, que apenas sugiere su existencia, en la esquina inferior derecha alcanza un tamaño notoriamente mayor al tiempo $T=50$, no reportado, mientras que el círculo central en el vórtice primario, el cual gira en sentido negativo, se inclina hacia la derecha y crece, haciendo espacio para un quinto contorno que aparece en el tiempo $T=86$, tampoco reportado, además, el número de subvórtices se incrementa a seis, debido a que el que se encuentra en la esquina superior izquierda se divide en dos, mientras que un vórtice terciario aparece en la esquina inferior izquierda, este subvórtice tendrá un papel especial en la evolución del flujo, pues como veremos aparece y desaparece en tiempos posteriores. Al tiempo $T=121.884$, Fig. 3.22 , se observa que existen cinco
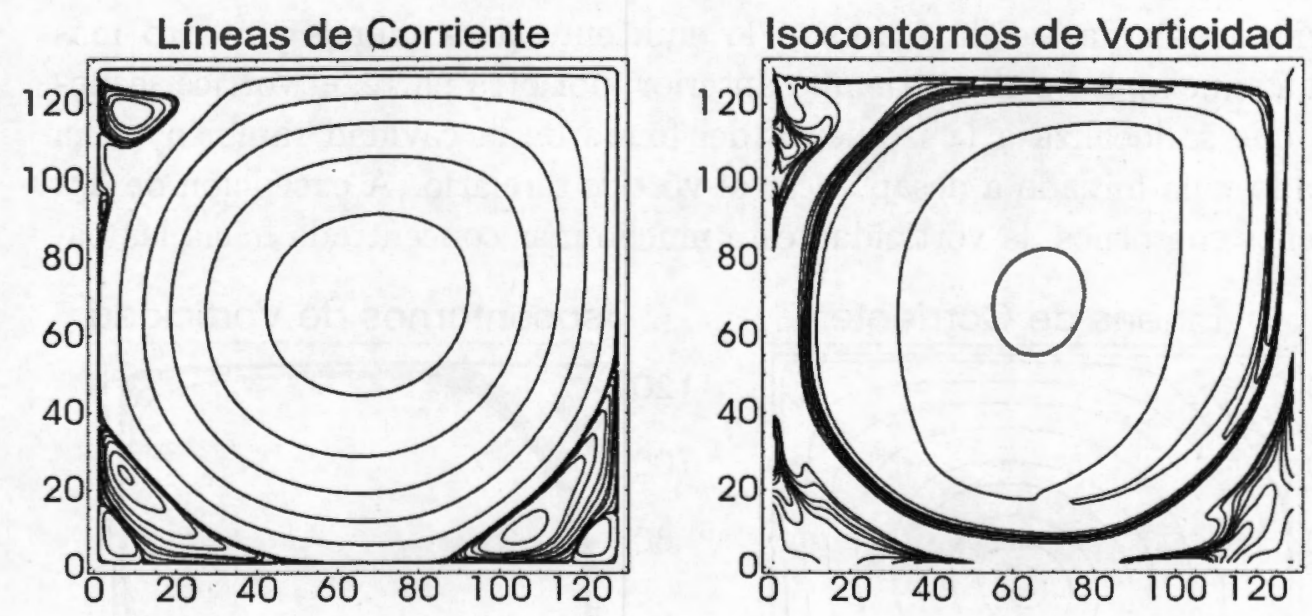

Fig. 3.22: $R e=10000, h=\frac{1}{128}, \Delta t=0.004, T=121.884$

contornos en el vórtice primario y que el número de subvórtices aún es de seis, dos de los cuales son terciarios y se localizan en las esquinas inferiores, el de la esquina izquierda no aparece en $T=25$ mientras que el de la esquina derecha, que apenas se apreciaba en ese tiempo inicial, ha aumentado su tamaño; por otro lado, los contornos de vorticidad muestran ya mayor concentración hacia las paredes de la cavidad.

La Figura 3.23 muestra el flujo al tiempo $T=168$, en donde dos nuevos contornos aparecen en el vórtice primario. El vórtice secundario, en la parte 

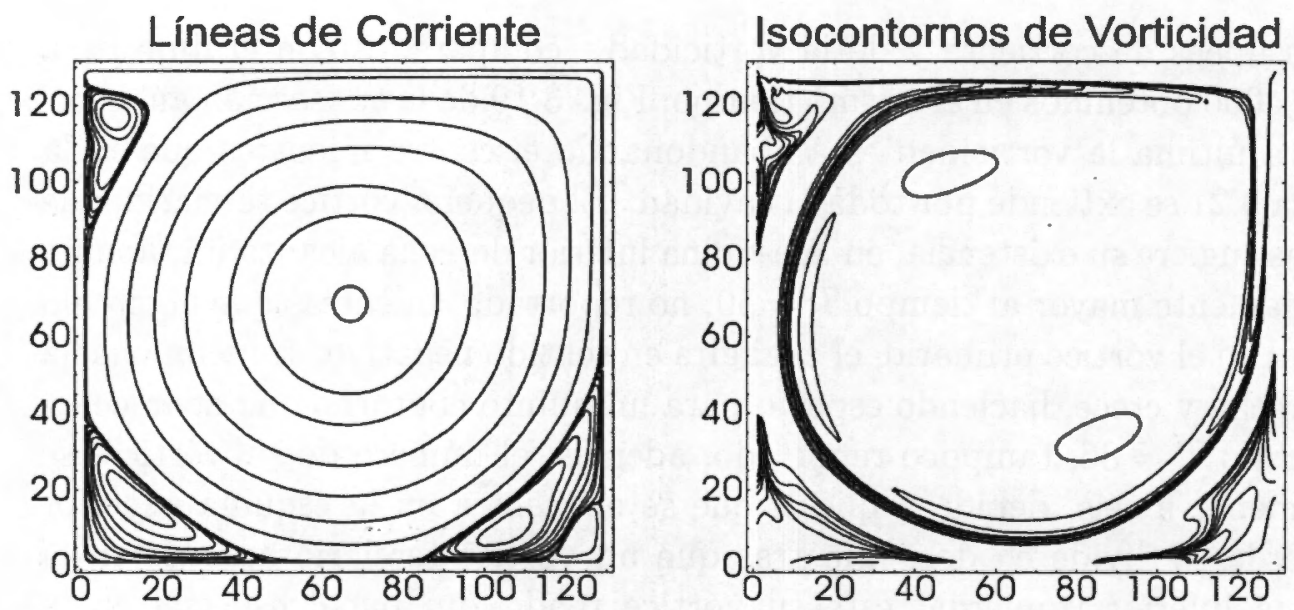

Fig. 3.23: $R e=10000, h=\frac{1}{128}, \Delta t=0.004, T=168$

superior izquierda, se ha alargado lo suficiente para unirse con otro más pequeño que aparece en un tiempo anterior; por otra parte, el vórtice secundario que se localiza a la izquierda del fondo de la cavidad también se ha alargado y ha forzado a desaparecer al vórtice terciario. A excepción de dos pequeños contornos, la vorticidad está mucho más concentrada hacia las pa-
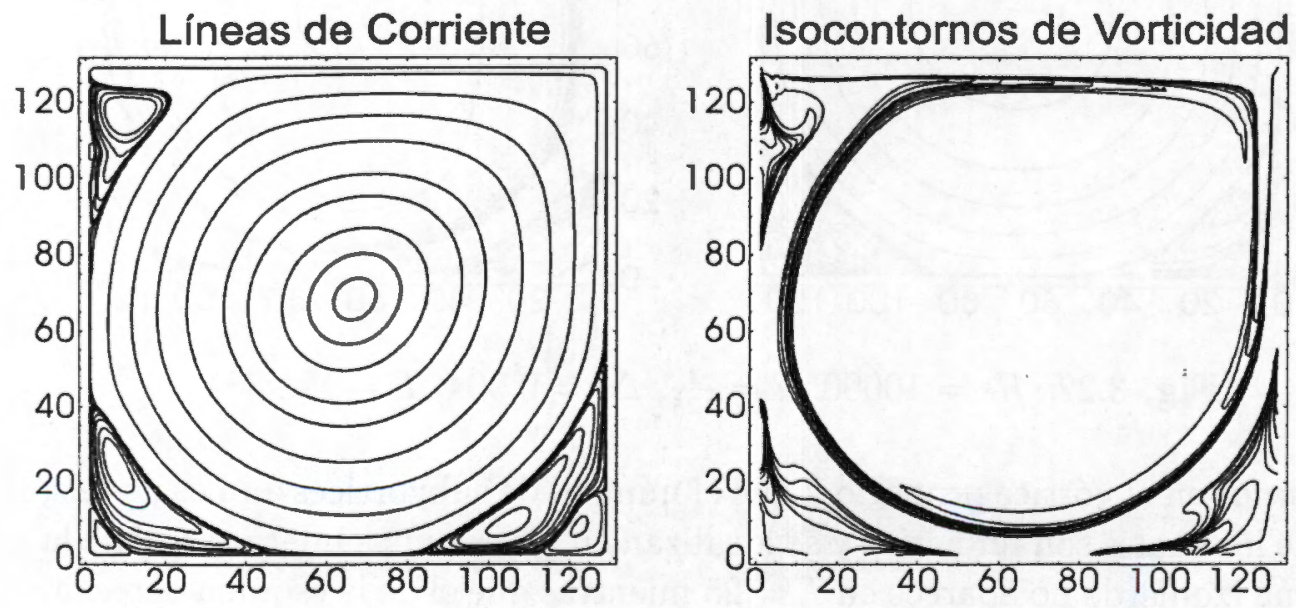

Fig. 3.24: $R e=10000, h=\frac{1}{128}, \Delta t=0.004, T=208$

redes. Al tiempo $T=208$, Fig. 3.24, se muestra un flujo que concuerda bien con el que presentan Erturk et al. [49] en donde, como se mencionó con anterioridad, emplean mallas más finas; el número de contornos en el 
vórtice primario se ha incrementado a nueve $\mathrm{y}$, sorpresivamente, el vórtice terciario del fondo que se había perdido, ha vuelto a aparecer. Además, comparando los respectivos flujos de las Figuras 3.14 y 3.15 con el de la Figura 3.24 al tiempo $T=208$, puede observarse que las líneas de corriente y los isocontornos de vorticidad tienen una forma definida, y éstos últimos tiende a confinarse dentro de la región de capa límite. La estructura de las líneas de corriente y de los contornos de vorticidad se conserva hasta $T=250$, no reportado, con excepción del vórtice secundario izquierdo en la parte superior que se ha dividido otra vez en dos, adquiriendo una forma similar a la de la Figura 3.22, correspondiente al tiempo $T=121.884$, mientras que los círculos internos del vórtice primario han dejado el centro de la cavidad hasta adquirir la forma que se observa al tiempo $T=2500$ en la Figura 3.25 , podemos percatarnos además de que el vórtice terciario en la parte izquierda
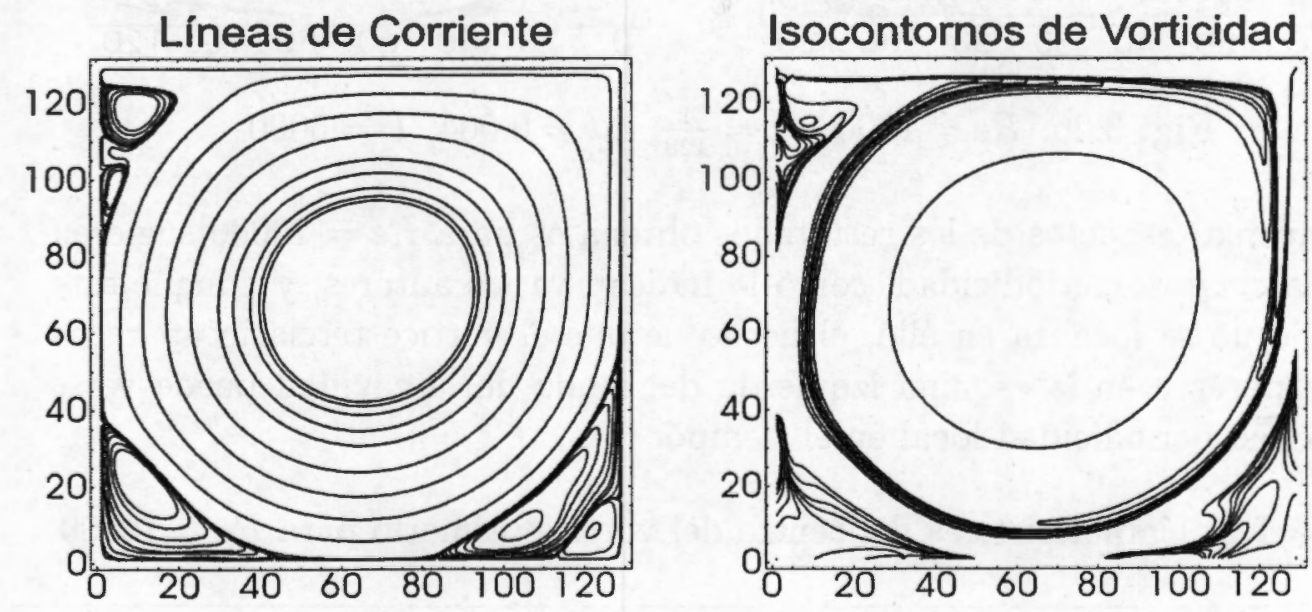

Fig. 3.25: $R e=10000, h=\frac{1}{128}, \Delta t=0.004, T=2500$

del fondo nuevamente se ha perdido; respecto a la vorticidad, aparece un contorno alrededor del centro de la cavidad, similar al de la Figura 3.22 en un tiempo anterior. Con objeto de determinar lo que sucede con la expansión, y creación, de los contornos en el vórtice primario, se continuó con la evolución del flujo hasta el tiempo $T=5000$, Fig. 3.26; el flujo es muy parecido al que se obtiene en $T=2500$, sólo que con pequeños cambios en los subvórtices, y nuevamente aparece el vórtice terciario en la parte inferior izquierda. Puede apreciarse también que la expansión de los contornos en el vórtice primario tiende a estacionarse y además no aparecen otros nuevos, así, el número de nueve contornos, reportados en Ghia et al. [48], al menos hasta $T=5000$ es 
un invariante, mostrando que las grandes estructuras, en este caso el vórtice primario, no cambia a partir de algún tiempo.
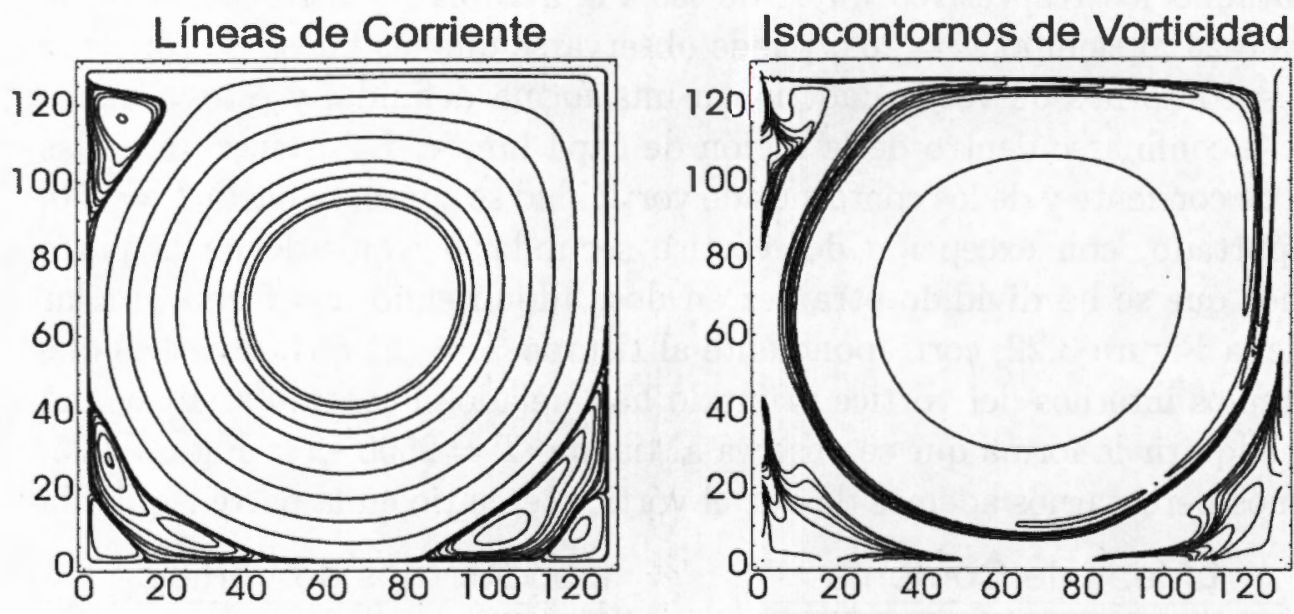

Fig. 3.26: $R e=10000, h=\frac{1}{128}, \Delta t=0.004, T=5000$

Diversos aspectos de los resultados obtenidos para $R e=10000$ sugieren alguna clase de periodicidad, como lo indican varios autores, y aunque este trabajo no se focaliza en ello, el hecho de que el vórtice terciario aparezca y desaparezca en la esquina izquierda del fondo de la cavidad puede verse como una periodicidad local en el tiempo.

Tabla I.2. Caracteristicas del centro del vórtice primario para $R e=10000$.

\begin{tabular}{|lccccc|}
\hline & $\mathrm{h}$ & $\mathrm{x}$ & $\mathrm{y}$ & \multicolumn{1}{c}{$\psi_{\min }$} & $|\omega|$ \\
\hline Ghia & $\frac{1}{256}$ & 0.5117 & 0.5333 & -0.119731 & 1.88082 \\
Erturk & $\frac{1}{600}$ & 0.5117 & 0.5300 & -0.120403 & 1.88899 \\
& & & & & \\
Este trabajo & $\frac{1}{128}$ & 0.5234 & 0.5313 & -0.118158 & 1.54997 \\
\hline
\end{tabular}

En la Tabla I.2 se muestran valores comparativos para $R e=10000$ en el centro del vórtice primario entre los obtenidos en Ghia et al. [48] y en Erturk et al. [49] y los que presentamos aquí al tiempo $T=208$, Fig. 3.24. Las discrepancias son: menos de $3 \%$ para la coordenada $x$; menos de $1 \%$ para la coordenada $y$; menos de $2 \%$ para $\psi_{\min }$ y a lo más $22.9 \%$ para $\omega$. Aunque la 
discrepancia en los valores de la vorticidad es significativa, los valores para los otro parámetros muestran buena similitud.

Finalizamos esta subsección señalando, por un lado, que se obtuvieron resultados para $R e=10000$ a tiempos cercanos al inicial $T=0$, cuando el flujo apenas inicia su desarrollo, $T=2,4,6,8,10 y 12$, como en la referencia Sahin y Owens [26], sin ninguna dificultad y los resultados correspondientes mostraron buena concordancia. Por otro lado, con el objeto de realizar una comparación con el flujo obtenido para $R e=10000$ a los tiempos $t=25$ y $T=208$, Figuras 3.21 y 3.24 , consideramos un número de Reynolds más grande, $R e=15000$, con $h=\frac{1}{256}$ y $\Delta t=0.0025$. En el flujo obtenido en $T=25$, Fig. 3.27, se observa que de manera similar a lo que sucede para $R e=10000$ en relación a valores más pequeños de $R e$, se ha incrementado el número de pequeñas estructuras en las líneas de corriente, ahora hay más de cuatro subvórtices, uno de los cuales es apenas visible abajo de otro mayor en la esquina superior izquierda, el vórtice terciario de la esquina derecha del fondo es más grande
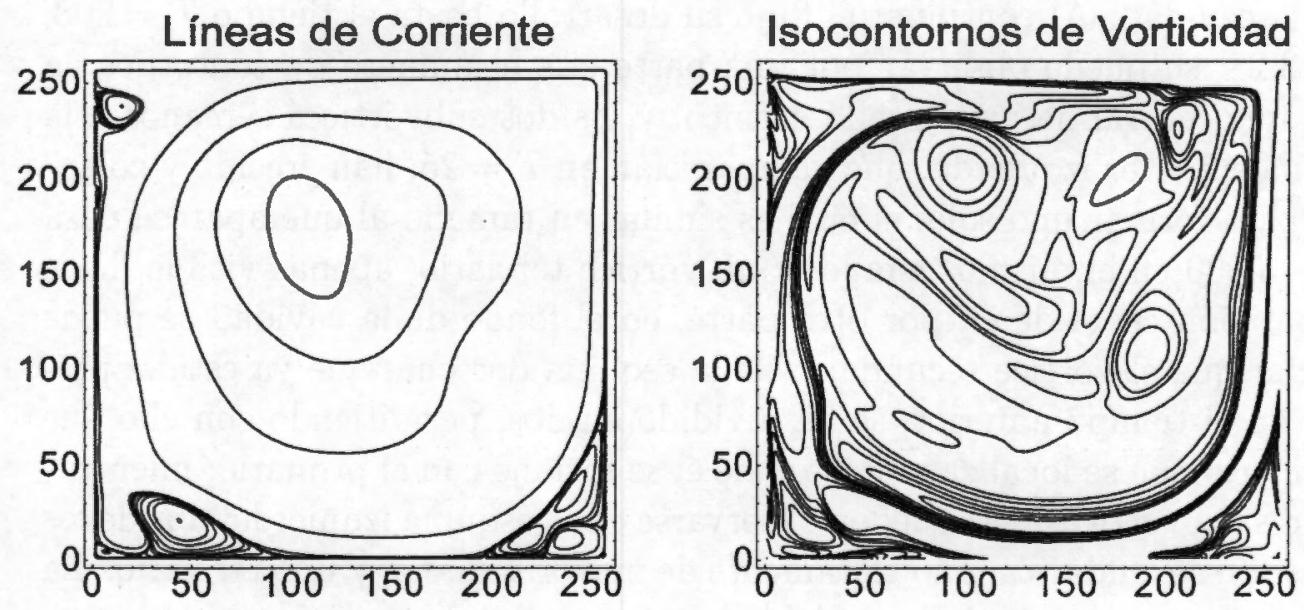

Fig. 3.27: $R e=15000, h=\frac{1}{256}, \Delta t=0.0025, T=25$

que el que se observa para $R e=10000$, y debajo de éste aparece, por primera vez, un vórtice cuaternario que es aún menos visible. Por otro lado, el vórtice primario en las líneas de corriente parece que, cualitativamente, ha girado un poco más, en dirección negativa, que el correspondiente de la Figura 3.21, lo cual podría verse como una consecuencia del movimiento más intenso del fluido para $R e=15000$; por su parte, los contornos de vorticidad al igual que 

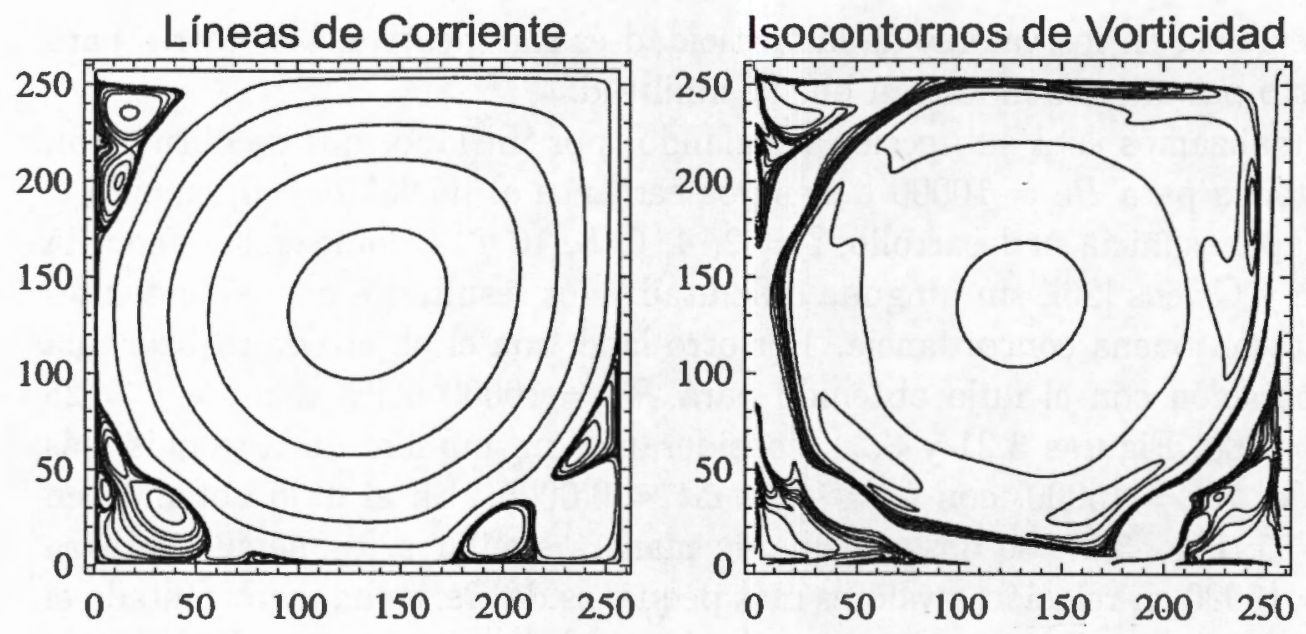

Fig. 3.28: $R e=15000, h=\frac{1}{256}, \Delta t=0.0025, T=208$

para el caso de $R e=10000$, en el mismo tiempo, también se extienden por toda la cavidad. Al continuar el flujo su desarrollo hasta el tiempo $T=208$, Fig. 3.28, se puede observar por una parte que el número de contornos en el vórtice central ha aumentado a cinco, y los dos subvórtices cercanos a la esquina superior izquierda, que ya aparecían en $T=25$, han crecido y colapsado para formar uno solo, el cual es similar en tamaño al que aparece para $R e=10000$ en el mismo tiempo, y un vórtice terciario, apenas visible, hace su aparición cerca de él; por otra parte, en el fondo de la cavidad se puede apreciar que el vórtice secundario de la esquina derecha, que ya estaba presente en el tiempo anterior, se ha dividido en dos, permitiendo con ello que el terciario que se localizaba debajo de él se fusione con el primario; mientras que tres subvórtices más pueden observarse en la esquina izquierda, dos de los cuales son secundarios, uno claramente de mayor tamaño, y uno terciario. La gráfica correspondiente a la vorticidad muestra el centro casi despejado, pero los contornos aún no se encuentran del todo definidos en su desarrollo. Así, el flujo observado en estos dos tiempos, para $R e=15000$, muestra algunos rasgos similares al presentado para $R e=10000$ cuando el flujo comienza a desarrollarse, es decir, al avanzar el tiempo, algunos subvórtices se dividen, otros surgen y/o desaparecen, variando con ello su número, mientras que el número de líneas de corriente presentes en el vórtice primario se incrementa ligeramente, además de que los isocontornos de vorticidad tienden a abandonar el centro para dirigirse hacia la frontera. 
4) Flujos con razón geométrica $A \geq 3$ para $R e=1000$ :

Respecto a los flujos que se presentan en esta sección, se observó que al aumentar la razón geométrica $\mathrm{A}$, éstos evolucionan lentamente, es decir, el flujo cambia muy poco entre dos pasos de tiempo consecutivos. Por esta razón consideramos conveniente emplear, para este caso, el criterio de convergencia relativo dado por

$$
\frac{\left\|f_{h_{x}, h_{y}}^{n+1}-f_{h_{x}, h_{y}}^{n}\right\|_{\infty}}{\left\|f_{h_{x}, h_{y}}^{n+1}\right\|_{\infty}}
$$

Con base en ello, las Figuras 3.29 y 3.30, muestran los resultados de estado estacionario para $R e=1000$ con razones geométricas $A=3$ (con tol $=10^{-9}$ ) y $A=4$ (con tol $=10^{-12}$ ) respectivamente.
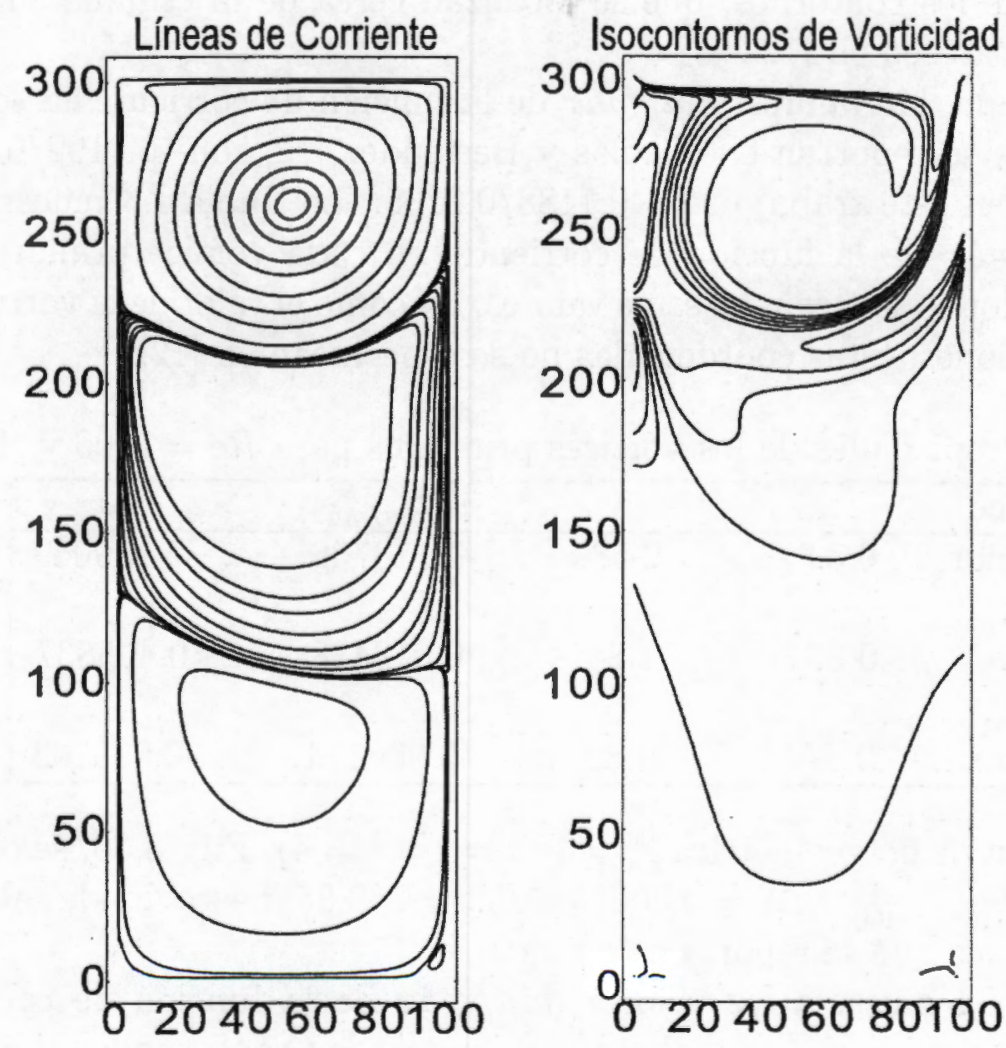

Fig. 3.29: $R e=1000, A=3, h_{x} \times h_{y}=\frac{1}{100} \times \frac{3}{300}, \Delta t=0.0001, T_{e s t}=122.85$

El flujo cuando $A=3(a=1$ y $b=3)$ se obtiene con $h_{x} \times h_{y}=\frac{1}{100} \times \frac{3}{300}$ y 
$\Delta t=0.0001$ en $T_{\text {est }}=122.85$; esta malla es significativamente más gruesa que la que se usa en Nicols y Bermúdez [12] en donde emplean $h_{x} \times h_{y}=\frac{1}{320} \times \frac{3}{960}$, la cual fue determinada a partir de estudios de independencia del paso de tiempo y de tamaño de malla con objeto de justificar que el flujo es correcto. Como se muestra en la Figura 3.29, se forman tres vórtices, en donde el superior y el medio tienen nueve contornos (este número coincide con el número de contornos en el vórtice primario para $A=1$, Fig. 3.13 , mientras que el inferior sólo tiene cuatro; además, aparece un pequeño vórtice secundario en la esquina inferior derecha. Aunque la diferencia entre los tamaños de las mallas es significativa, los resultados concuerdan con el reportado en Nicolás y Bermúdez [12], la única diferencia es que el pequeño vórtice secundario que aparece, en ese trabajo, en la esquina inferior izquierda. También se observa una buena coincidencia con la vorticidad, tomando en consideración que algunos de los contornos, que se localizan cerca de la esquina superior derecha, no aparecen en [12].

Por otro lado, los valores mín/máx de la función de corriente en toda la cavidad $\bar{\Omega}$ que se reportan en Nicolás y Bermúdez [12] son $-0.1192 / 0.0135$, mientras que en este trabajo son $-0.1188 / 0.0135$. La Tabla I.3 muestra los valores mín/máx de la función de corriente, en cada vórtice primario, las coordenadas donde se alcanzan estos valores, así como el valor de la vorticidad en estas posiciones; estas coordenadas no se especifican en [12].

Tabla I.3. Propiedades de los vórtices primarios para $R e=1000$ y $A=3$.

\begin{tabular}{|lcccc|}
\hline Vórtice & $\mathrm{x}$ & $\mathrm{y}$ & $\psi_{(\min / \operatorname{máx})}$ & $\omega$ \\
\hline Superior & 0.54 & 2.58 & -0.118756 & -2.139049 \\
Medio & 0.35 & 1.84 & 0.013438 & 0.465837 \\
& & & & \\
Inferior & 0.47 & 0.82 & -0.000174 & -0.006763 \\
\hline
\end{tabular}

El flujo con razón geométrica $A=4(a=1$ y $b=4)$, Fig. 3.30, se obtuvo con $h_{x} \times h_{y}=\frac{1}{100} \times \frac{4}{400}$ y $\Delta t=0.0001$ en $T_{\text {est }}=259.85$; hasta donde sabemos es la primera vez que se reporta este flujo.

La Tabla I.4 muestra los valores mín/máx de la función de corriente en cada vórtice primario, que ahora son cuatro, las coordenadas donde se alcanzan estos valores, así como el valor de la vorticidad en estas posiciones. Como se observa de esta misma tabla al restar una unidad a la coordenada Y de los primeros tres vórtices, se obtienen los valores correspondientes a la 

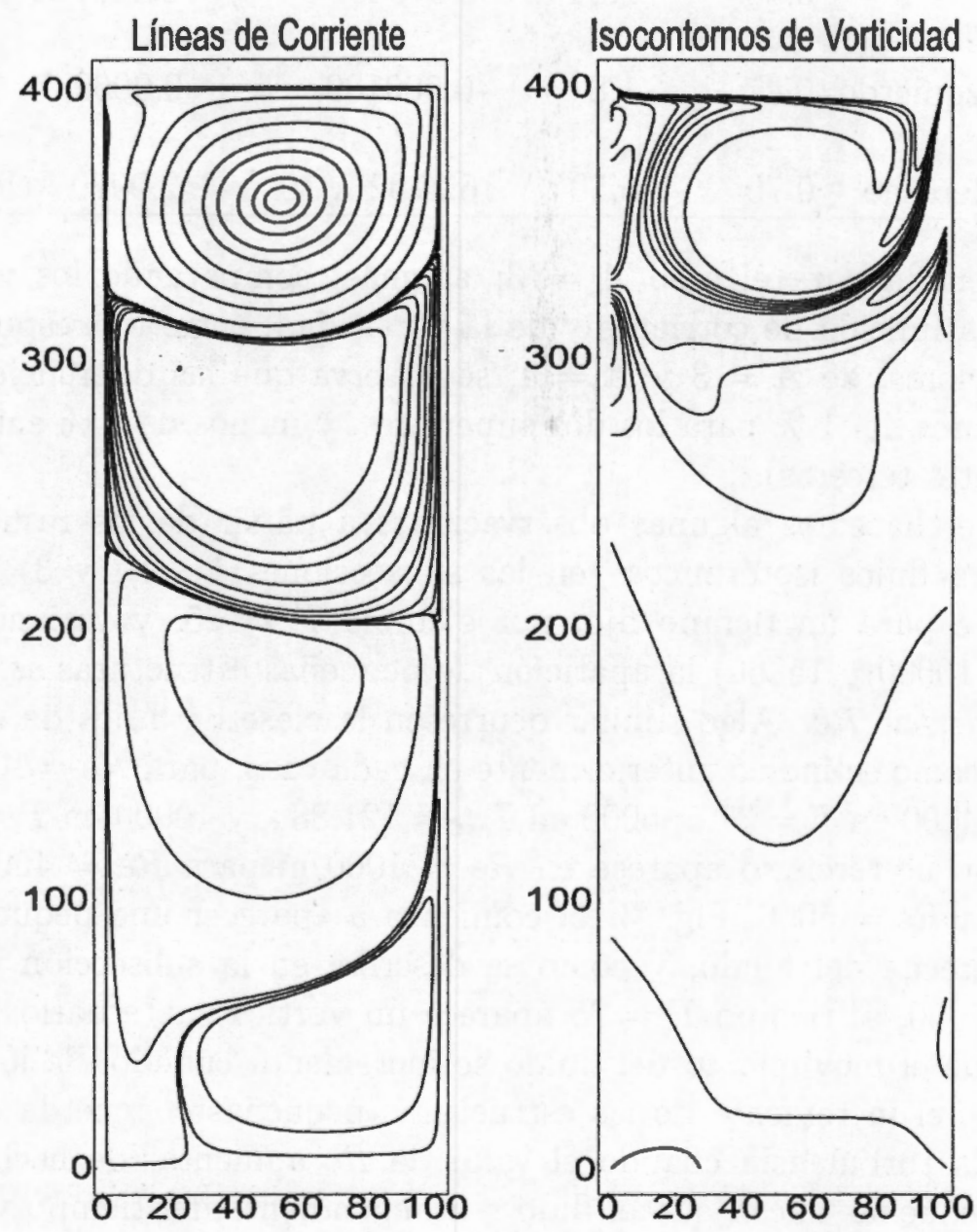

Fig. 3.30: $R e=1000, A=4, h_{x} \times h_{y}=\frac{1}{100} \times \frac{4}{400}, \Delta t=0.0001, T_{e s t}=259.85$ 
Tabla I.4. Propiedades de los vórtices primarios para $R e=1000$ y $A=4$.

\begin{tabular}{|lccll|}
\hline Vórtice & $\mathrm{x}$ & $\mathrm{y}$ & $\psi_{(\min / \max )}$ & $\omega$ \\
\hline Superior & 0.54 & 3.58 & -0.118762 & -2.139292 \\
Medio & 0.35 & 2.84 & 0.013461 & 0.465748 \\
Inferior izquierdo & 0.47 & 1.82 & -0.000189 & -0.006987 \\
Inferior derecho & 0.70 & 0.50 & $6.1792 \times 10^{-7}$ & $2.9970 \times 10^{-5}$ \\
\hline
\end{tabular}

misma coordenada para el caso $A=3$; además, comparando los valores extremos de la función de corriente y de la vorticidad, entre los respectivos vórtices superiores, de $A=3$ y $A=4$, se observa que las diferencias son pequeñas (menos del $1 \%$ para los dos superiores, y menos de $9 \%$ entre los correspondientes terceros).

Finalmente, hacemos algunas observaciones a partir de los resultados obtenidos para flujos isotérmicos: en las subsecciones 1), 2), y 3) puede observarse que para un tiempo fijo, por ejemplo $T=25$, y variando $R e$ $(R e=4000,10000$ y 15000) la aparición de pequeñas estructuras se incrementa al aumentar Re. Algo similar ocurre en la clase de flujos de estado estacionario, como se indicó anteriormente en cada caso, para $R e=1000$ en $T_{\text {est }}=31.15 ; 4000$ en $T=37.5 ; 5000$ en $T_{\text {est }}=121.884 ;$ y 10000 en $T=208$; ningún subvórtice terciario aparece en $R e=1000$ ni para $R e=4000$, sin embargo, para $R e=5000$, Fig. 3.15 , comienza a aparecer uno pequeño en la esquina derecha del fondo, y como se describe en la subsección previa para $R e=15000$, al tiempo $T=25$ aparece un vórtice cuaternario. Todo esto indica que el movimiento del fluido se incrementa cuando $R e$ lo hace. Por otro lado, el incremento de las estructuras pequeñas concuerda con la transición a la turbulencia cuando el valor de Re aumenta; de hecho, las estructuras pequeñas hacen que el flujo sea dependiente del tiempo ya que la interacción con las estructuras grandes, en este caso el vórtice primario con los vórtices secundarios y de éstos con los terciarios, tiende a incrementar su comportamiento oscilatorio. En relación con esto cabe recordar que para $R e=10000$ la estructura grande dada por el vórtice primario puede no mostrar cambios a partir de algún tiempo ya que, como se observó, la cantidad de nueve contornos ya no cambia a partir de $T=208$; sin embargo, la interacción de esta estructura con las más pequeñas puede implicar una periodicidad local en el tiempo, como se puntualizó en la subsección 3 (Flujos 
dependientes del tiempo).

Respecto al caso de $R e=1000$ con $A \geq 3$ cabe señalar, a partir de las líneas de corriente y de los isocontornos de vorticidad correspondientes, que la mayor actividad del flujo tiene lugar en la parte superior y media de la cavidad; para $A=4$ la región donde existe menor actividad se divide en dos partes. Por el contrario, para el flujo con $A=2$ la actividad más alta se distribuye de manera casi uniforme en la parte superior e inferior de la cavidad, donde se localizan los dos vórtices primarios; este resultado se reporta en Goyon [11] y Nicolás y Bermúdez [12] pero no en el presente trabajo, debido a que es común que otros autores lo reporten, en su lugar presentamos el caso con $A=4$ que, como ya se señaló, parece ser la primera vez que se reporta. Por otra parte, debido a que para los flujos con $A=3$ y $A=4$ no fue posible determinar $T_{\text {est }}$ con el criterio de convergencia inicialmente propuesto (criterio de paro absoluto), consideramos conveniente emplear, sólo para este caso, el correspondiente criterio relativo de convergencia (o de paro) con el objeto de determinar el tiempo $T_{\text {est }}$ en que ambos flujos alcanzan el estado estacionario.

\subsection{Esquema numérico en medios porosos}

El esquema que se desarrolla para aproximar la solución del sistema (2.12) en medios porosos sigue de cerca algunos de los pasos del procedimiento numérico empleado para medios libres. Si bien la ecuación de energía térmica y la de continuidad (condición de incompresibilidad) tienen la misma forma en ambos medios, la ecuación de momento es claramente distinta, y en el caso de medios porosos, el término de la derivada temporal de la velocidad, que juega un papel fundamental en la aplicación de los métodos de descomposición de operadores, no existe. Es por ello que los pasos del esquema numérico que involucran a las ecuaciones de momento y continuidad muestran una forma distinta a los que se obtienen en medios libres.

En el sistema (2.12) la derivada temporal, en la ecuación de energía, se sustituye nuevamente a través de la aproximación dada por (3.1), con lo cual 
el correspondiente sistema semidiscreto en tiempo adquiere la siguiente forma

$$
\begin{gathered}
\frac{\frac{3}{2} \theta^{n+1}-2 \theta^{n}+\frac{1}{2} \theta^{n-1}}{\Delta t}-\nabla^{2} \theta^{n+1}+\left(\mathbf{u}^{n+1} \cdot \nabla\right) \theta^{n+1}=0 \\
\mathbf{u}^{n+1}+\nabla p^{n+1}=R a_{p} \theta^{n+1} \mathbf{e}_{2} \\
\nabla \cdot \mathbf{u}^{n+1}=0
\end{gathered}
$$

De manera similar al caso de medios libres, en el procedimiento numérico resolvemos la ecuación de energía en el primer paso, donde el término convectivo $\mathbf{u} \cdot \nabla \theta$ se aproxima a través de la extrapolación lineal dada por (3.4). Posteriormente, al aplicar la divergencia a la ecuación de momento y considerar la condición de incompresibilidad, obtenemos en el segundo paso una ecuación de tipo Poisson para la presión; al tomar la componente normal de la respectiva ecuación semidiscreta de momento, sobre la frontera de la región, se obtiene la correspondiente condición de frontera de tipo Neumann, en donde la velocidad $\mathbf{u}^{n+1}$ se aproxima con una extrapolación lineal de los valores correspondientes en los dos niveles previos de tiempo. Finalmente, en el último paso, se obtiene la velocidad $\mathbf{u}^{n+1}$. De esta manera el esquema completo se reduce a tres pasos:

$$
\begin{aligned}
& \text { Paso 1) } \quad\left(\nabla^{2}-\frac{3}{2 \Delta t}\right) \theta^{n+1}=-\frac{2 \theta^{n}}{\Delta t}+\frac{\theta^{n-1}}{2 \Delta t}+2\left(\mathbf{u}^{n} \cdot \nabla\right) \theta^{n} \\
& -\left(\mathbf{u}^{n-1} \cdot \nabla\right) \theta^{n-1} \quad \text { en } \Omega \text {, } \\
& \left.B \theta^{n+1}\right|_{\Gamma}=0 \\
& \text { Paso 2) } \quad \nabla^{2} p^{n+1}=R a_{p} \nabla \cdot\left(\theta^{n+1} \mathbf{e}_{2}\right) \quad \text { en } \Omega \text {, } \\
& \left.\frac{\partial p^{n+1}}{\partial \mathbf{n}}\right|_{\Gamma}=\mathbf{n} \cdot\left[R a_{p} \theta^{n+1} \mathbf{e}_{2}-2 \mathbf{u}^{n}+\mathbf{u}^{n-1}\right] \\
& \text { Paso 3) } \quad \mathbf{u}^{n+1}=R a_{p} \theta^{n+1} \mathbf{e}_{2}-\nabla p^{n+1} \quad \text { en } \Omega \text {, } \\
& \mathbf{u}^{n+1} \mid \mathbf{r}=\mathbf{g} \text {, }
\end{aligned}
$$

En este caso, debemos resolver sólo dos problemas elípticos en cada nivel de tiempo, uno para la temperatura $\theta^{n+1}$ en el paso 1) y el segundo para la presión $p^{n+1}$ en el paso 2), mientras que la velocidad vectorial $\mathbf{u}^{n+1}$ se obtiene en forma explícita en el paso 3). Los valores de $\theta^{1}, p^{1}$ y $\mathbf{u}^{1}$ se obtienen 
procediendo de manera similar a como se indicó en el caso de medios libres, con el presente esquema.

Como se observa, el procedimiento numérico que obtenemos es, al igual que el correspondiente en medios libres, independiente de la dimensión del espacio y de la forma de la región. Las derivadas espaciales de $\theta, p$ y $\mathbf{u}$ se aproximan también mediante los esquemas de diferencias finitas de segundo orden descritos ya en el caso de medios libres.

\subsection{Resultados numéricos}

Los resultados numéricos que se presentan en esta sección muestran la evolución en el desarrollo de los flujos correspondientes a $R a_{p}=10^{2}$ y $10^{3}$, a través de distintos estados transitorios hasta llegar al estacionario, en la cavidad cuadrado unitaria; la condición de frontera para la velocidad es $\mathbf{u}=\mathbf{0}, \mathbf{y}$ para la temperatura se tiene nuevamente

$$
\left.\frac{\partial \theta}{\partial n}\right|_{y=0,1}=\left.0 \quad \theta\right|_{x=0}=\left.0.5 \quad \theta\right|_{x=1}=-0.5,
$$

como se supone que el fluido parte del reposo, la respectiva condición inicial para la velocidad es $\mathbf{u}(\mathbf{x}, 0)=\mathbf{0}$, mientras que para la temperatura se tiene que $\theta(x, 0)=0$. La tolerancia empleada para el criterio de convergencia es de $10^{-5}$. Nuevamente, los resultados se reportan en términos de las isotermas y de las líneas de corriente. Los valores que corresponden al paso de tiempo $\Delta t$ y al tamaño de la malla $h$, se indican en cada caso de estudio.

En la Figura 3.31 se muestra una serie de resultados a tiempos distintos para $R a_{p}=10^{2}$ y en donde se emplea una malla con $h=\frac{1}{30}$ y un paso de tiempo dado por $\Delta t=10^{-3}$. El tiempo en que se considera cada estadío se indica en la misma Figura, mientras que los valores correspondientes de $\psi_{\min }$ y $\overline{N u}$, para cada uno de ellos, se muestra en la Tabla R.1. Como se aprecia, al tiempo $T=0.01$, las isotermas son casi paralelas a las paredes laterales, lo cual es una característica cuando el mecanismo principal en la transferencia de calor es la conducción; al comenzar el calentamiento, las isotermas se encuentran muy cerca unas de otras a todo lo largo de la frontera caliente y, como puede apreciarse del valor correspondiente de $\overline{N u}$, se alcanza la más alta transferencia de calor global (en comparación con la de los otros tiempos reportados). Las líneas de corriente muestran que el fluido inicia su movimiento, cerca de la mitad de las paredes laterales, en pequeñas celdas que giran de izquierda a derecha, en sentido negativo; el flujo generado es 
Líneas de corriente
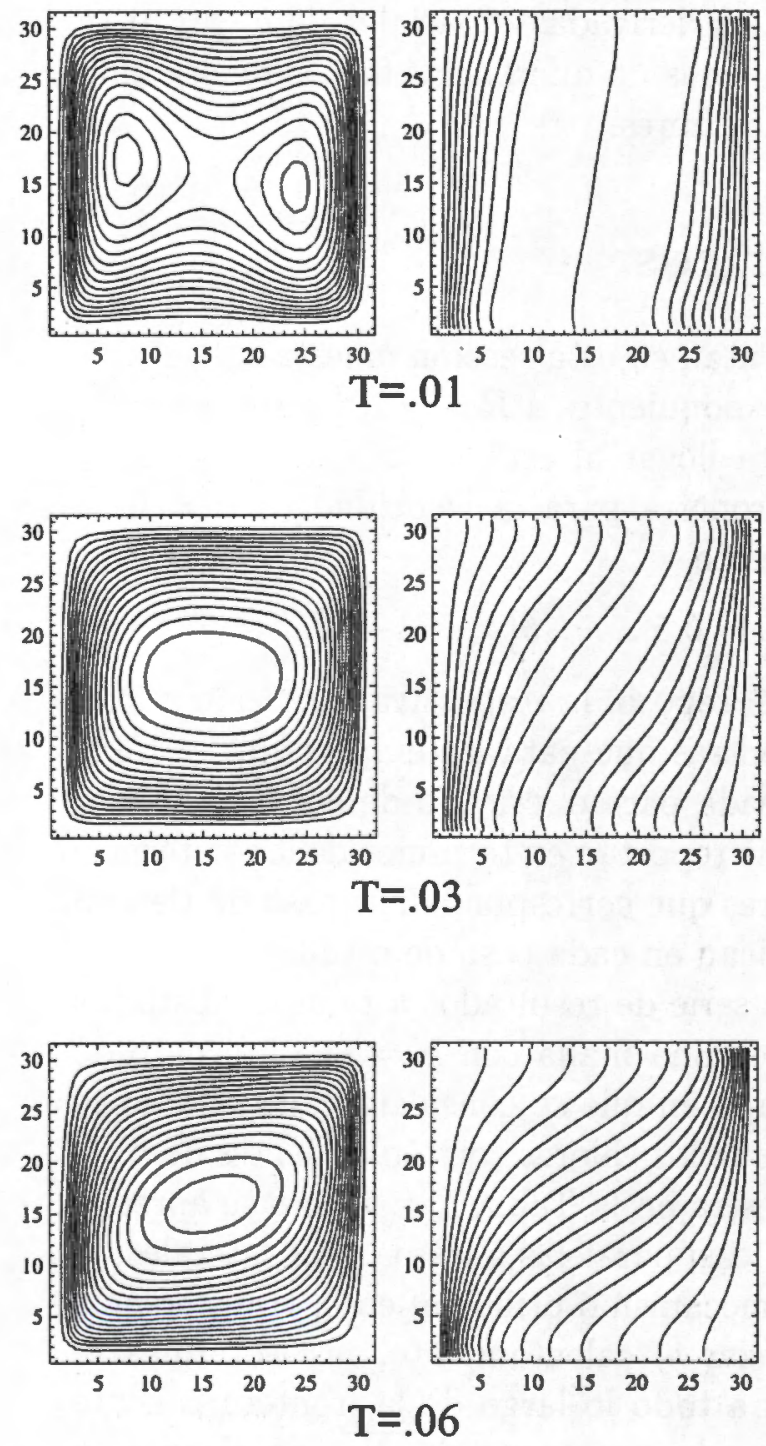

Líneas de corriente
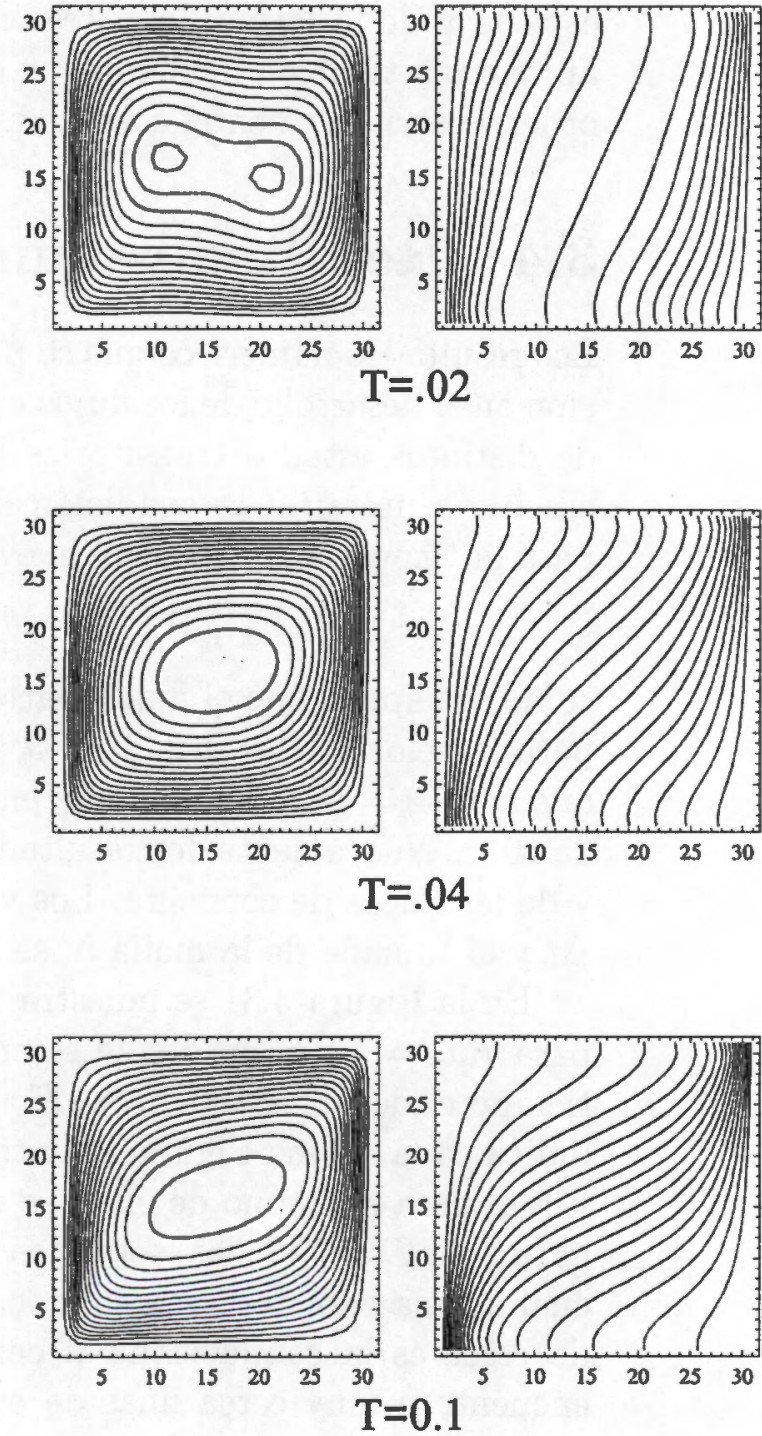

Fig. 3.31: $R a_{p}=10^{2}, h=\frac{1}{30}, \Delta t=10^{-3}$ 
lento, lo que se confirma con el respectivo valor mínimo de la función de corriente, $\psi_{\min }$. En un tiempo posterior, $T=0.02$, la conducción continúa presente, las isotermas empiezan a doblarse y aquellas que se encuentran cerca de la frontera caliente se han alejado un poco más de ésta, con lo que la transferencia de calor global $\overline{N u}$, disminuye; sin embargo el movimiento del fluido ha ganado intensidad, $\psi_{\min }$, y las pequeñas celdas que aparecían en el tiempo anterior han empezado a colapsar. Al continuar avanzando el tiempo, $T=0.03$, las isotermas han aumentado su distribución sobre la mitad de la cavidad, señalando que el fluido caliente se ha extendido más hacia la parte superior de la cavidad, muy cerca de la pared fría, con lo que la transferencia de calor sobre la pared caliente vuelve a disminuir un poco pero, a cambio, el movimiento del fluido aumenta; las dos pequeñas celdas, que habían empezado a colapsar, ya se han fusionadó por completo, dando origen a una única celda, que continúa extendiéndose en la cavidad. Cuando $T=0.04$, y el mecanismo de convección adquiere importancia, existe una mayor destribución del flujo, debido al ascenso y descenso de buena parte del fluido cerca de las paredes caliente y fría; el respectivo valor de $\psi_{\min }$ indica que el fluido sigue ganando fuerza pero ahora la transferencia de calor, $\overline{\mathrm{Nu}}$, muestra un ascenso leve.

Tabla R.1 Resultados correspondientes a $R a_{p}=10^{2} \operatorname{con} h=\frac{1}{30}$ y $\Delta t=10^{-3}$, para diferentes tiempos.

\begin{tabular}{|lcc|}
\hline \multicolumn{1}{|c}{$\psi_{\text {min }}$} & $\overline{N u}$ \\
\hline 0.01 & -3.0112 & 3.0693 \\
0.02 & -3.9100 & 2.4025 \\
0.03 & -4.5658 & 2.2044 \\
0.04 & -4.8175 & 2.2303 \\
0.06 & -4.6569 & 2.5799 \\
0.10 & -4.3597 & 2.8917 \\
0.21 (est.) & -4.3198 & 2.9310 \\
\hline
\end{tabular}

En $T=0.06$ puede apreciarse que el flujo se encuentra claramente desarrollado y la transferencia de calor por convección ya está presente, siendo ahora el mecanismo dominante; los valores correspondientes para $\psi_{\min }$ y $\overline{N u}$ señalan que a pesar de que el movimiento del fluido disminuye un poco, la transferencia de calor continúa aumentando. Esta situación se mantiene para $T=0.1$, así como para tiempos posteriores hasta el estado estacionario, en donde el movimiento del fluido sigue disminuyendo y la transferencia de calor aumenta 
ligeramente. En este último tiempo se tienen ya las características fundamentales del flujo de estado estacionario, el cual se alcanza en $T_{\text {est }}=0.21$, y cuyas gráficas respectivas son iguales a las que se observan para $T=0.1$, aunque, como se aprecia en la Tabla R.1, los respectivos valores de $\psi_{\min }$ y de $\overline{N u}$, muestran una pequeña variación. Los resultados correspondientes al estado estacionario concuerdan con los obtenidos por Baytas [29] en una cavidad inclinada cuando el ángulo de inclinación es $\phi=0^{\circ}$. Saeid y Pop [30] no presentan isotermas ni líneas de corriente para este valor de $R a_{p}$, sin embargo el correspondiente valor del número de Nusselt global que obtienen es $\overline{N u}=3.002$ mientras que en este trabajo $\overline{N u}=2.9310$, lo que indica una diferencia de $2.4 \%$.

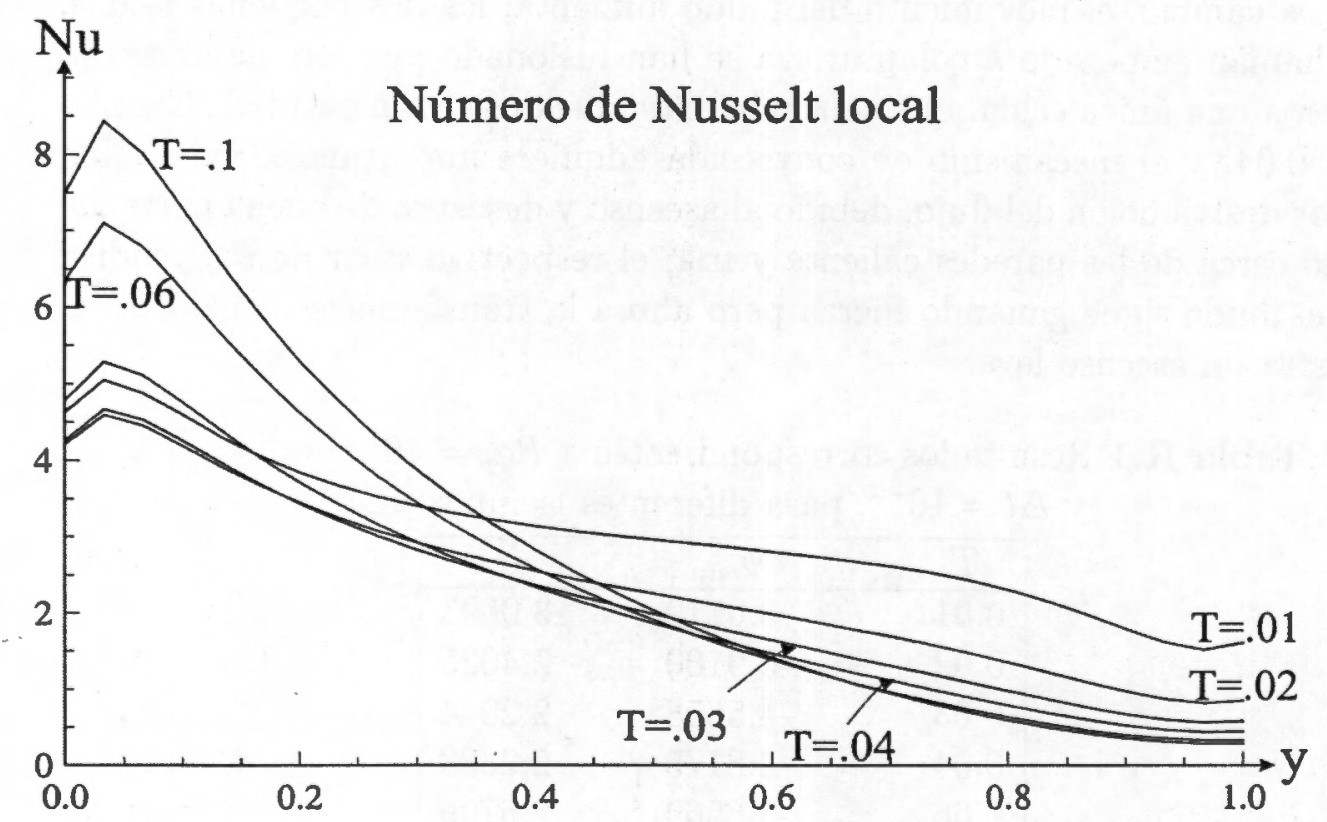

Fig. 3.32: $R a_{p}=10^{2}, h=\frac{1}{30}, \Delta t=10^{-3}$

Las gráficas del número de Nusselt local que se obtuvieron en los diferentes tiempos, Fig. 3.32, muestran que la transferencia de calor local sobre la frontera caliente es máxima cerca del fondo de la cavidad, donde se presenta la mayor concentración de isotermas, y disminuye paulatinamente con la altura; tanto el valor máximo como el descenso que muestran las gráficas se hace más pronunciado para $T$ grandes. Esto se debe a que inicialmente las isotermas son casi paralelas y la transferencia de calor local no cambia a 
lo largo de la frontera, sin embargo al avanzar el tiempo e irse alejando las isotermas cada vez más unas de otras, en la parte superior de la pared, esta transferencia va disminuyendo perceptiblemente con la altura; sin embargo para los últimos tiempos mostrados, y cuando la convección es claramente el mecanismo predominante del proceso, el fluido frío del fondo, proveniente de la frontera derecha, genera un mayor gradiente de temperatura en esta zona, lo que a su vez conduce a una transferencia de calor mucho más alta de la que se obtiene en tiempos anteriores. Por otra parte, las fluctuaciones que se observan alrededor de la segunda mitad de la correspondiente gráfica del número de Nusselt local para $T=0.01$, tienen relación con la presencia de la pequeña celda cercana a la frontera caliente, donde se está cuantificando la transferencia de calor; estas variaciones son apenas perceptibles en la respectiva gráfica para $T=0.02$ debido a que esta celda ha disminuido su tamaño.

Para $R a_{p}=10^{3}$ con $h=\frac{1}{70}$ y $\Delta t=10^{-6}$, se procede de manera similar al caso anterior, por lo que la Figura 3.33 muestra los resultados que se obtienen para tiempos diferentes, los cuales son los mismos que se consideran en Saeid y Pop [30] para este mismo valor de $R a_{p}$, con $h=\frac{1}{40}$ y $\Delta t=10^{-4}$. Al tiempo $T=0.0025$ las isotermas se concentran en las paredes laterales, así, el flujo comienza su desarrollo cerca de éstas, lo que nuevamente se manifiesta a través de las dos celdas secundarias que se observan en las líneas de corriente, ambas rotando en sentido negativo; los valores respectivos de $\psi_{\min }$ y $\overline{N u}$, pueden verse en la Tabla R.2. Al llegar a $T=0.005$ las isotermas abarcan una mayor porción de las fronteras superior e inferior indicando que el fluido caliente, en la parte superior, y el frío, en la inferior, continúan avanzando hacia las fronteras derecha e izquierda, respectivamente; además, las celdas secundarias que aparecen en el tiempo anterior se extienden un poco y algunos de estos contornos se unen en uno solo; si bien el valor de $\psi_{\min }$ señala un aumento en el movimiento del fluido, se aprecia que la transferencia de calor global, $\overline{\mathrm{Nu}}$ ha disminuído ligeramente. Cuando $T=0.01$, las isotermas se han extendido hasta ocupar gran parte de la cavidad, con excepción del centro de la misma, logrando aumentar el movimiento del fluido y, de manera notoria, la trasferencia de calor global; por su parte, las celdas secundarias han colapsado casi por completo. Al avanzar el tiempo hasta $T=0.02$ el fluido caliente se extiende ya por toda la parte superior de la cavidad, mientras que el frío lo hace en el fondo, y las líneas de corriente muestra ya una sola celda alargada hacia las fronteras laterales, y si bien ahora el fluido es un poco menos activo, la transferencia de calor muestra un importante incre- 
Líneas de corriente

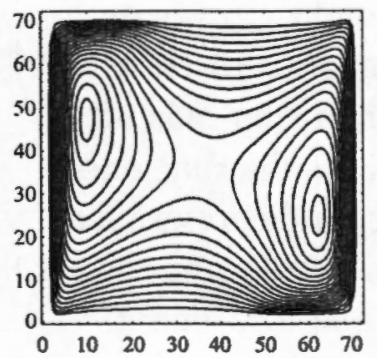

$$
\mathrm{T}=.0025
$$
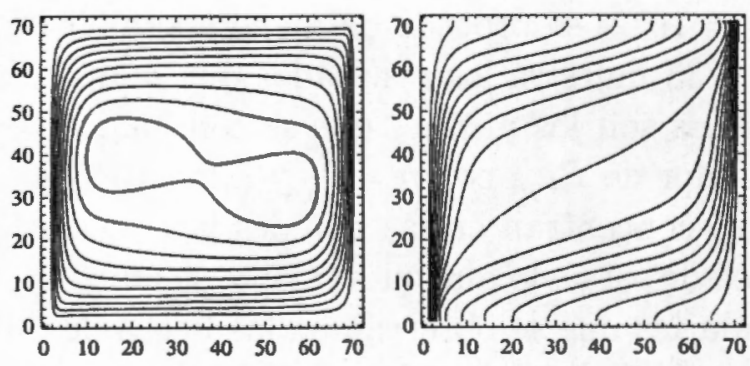

$\mathrm{T}=.01$
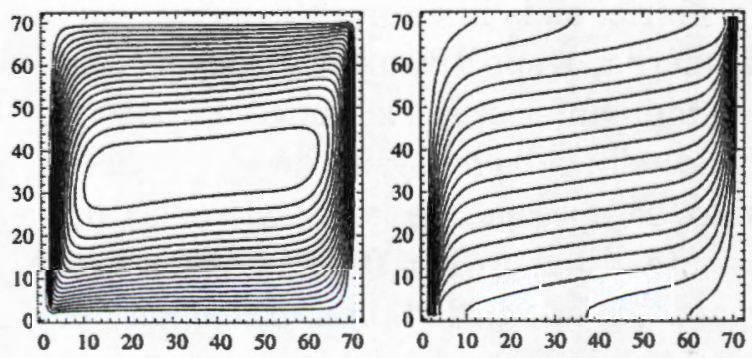

$\mathrm{T}=.04$
Líneas de corriente
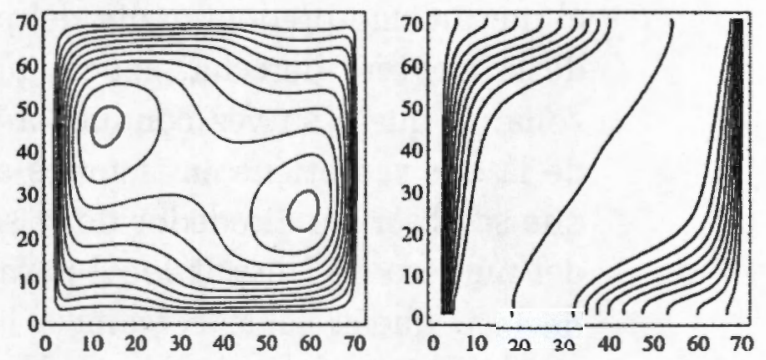

$\mathrm{T}=.005$
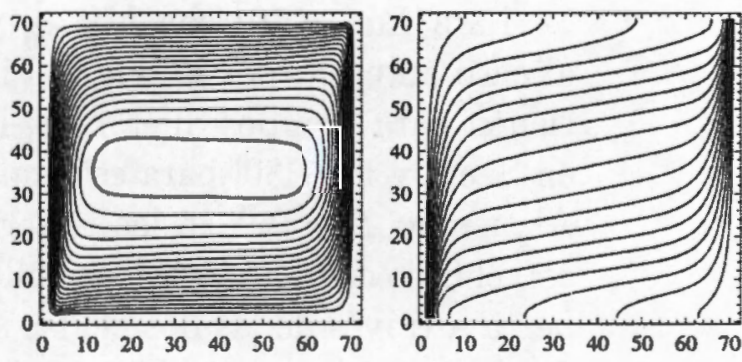

$\mathrm{T}=.02$
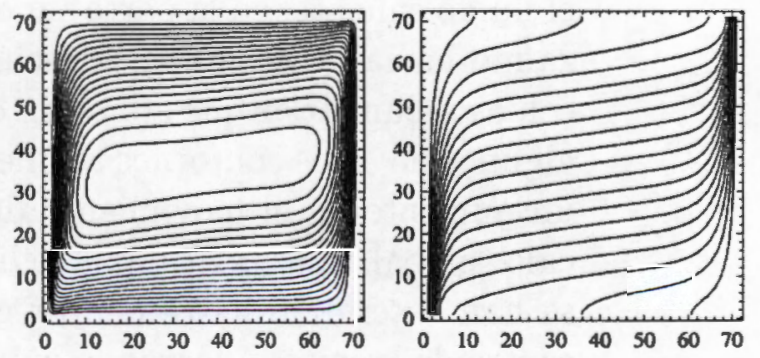

$\mathrm{T}=.08$

Fig. 3.33: $R a_{p}=10^{3}, h=\frac{1}{70}, \Delta t=10^{-6}$ 
mento; aunque todas estas características se conservan, en general, cuando $T=0.04$, puede observarse que la parte central de la celda, en las líneas de corriente, se extiende hacia las esquinas superior derecha e inferior izquierda. El flujo para $T=0.08$, tiempo en que Saeid y Pop [30] reportan que se alcanza el estado estacionario, ya no presenta cambios sustanciales, los resultados obtenidos para este último tiempo son prácticamente los mismos que aquellos correspondientes al estado estacionario, el cual se alcanza en $T_{e s t}=.0844$; éstos últimos guardan buen parecidos a los obtenidos por Baytas [29] con $\phi=0^{\circ}$, mientras que las isotermas y las líneas de corriente que se observan en los tiempos indicados del flujo transitorio, especialmente los correspondientes a los primeros tres tiempos $T=.0025, .005, .01$, muestran gran similitud con los obtenidos por Saeid y Pop [30]. Aunque los valores respectivos de $\psi_{\min }$ y $\overline{N u}$ para $T=0.08$ siguen el mismo comportamiento que en los dos tiempos anteriores, esta situación cambia en el estado estacionario, ya que el fluido aumenta ligeramente su actividad y la transferencia de calor disminuye un poco. Respecto al valor del número de Nusselt global que se alcanza en el estacionario, este es casi igual al valor $\overline{\mathrm{Nu}}=13.726$ que reportan Saeid y Pop.

Tabla R.2. Resultados correspondientes a $R a_{p}=10^{3}$ con $h=\frac{1}{70}$ y $\Delta t=10^{-6}$, para diferentes tiempos.

\begin{tabular}{|lcc|}
\hline \multicolumn{1}{|c}{$T$} & $\psi_{\min }$ & $\overline{N u}$ \\
\hline 0.0025 & -18.0334 & 8.7900 \\
0.005 & -20.4367 & 8.4166 \\
0.01 & -20.8907 & 10.0889 \\
0.02 & -19.2322 & 12.9148 \\
0.04 & -18.5146 & 13.7002 \\
0.08 & -18.4592 & 13.7872 \\
0.0844 (est.) & -18.4726 & 13.7203 \\
\hline
\end{tabular}

La gráfica del número de Nusselt local, Fig. 3.34, muestra un efecto similar al observado para el caso de $R a_{p}=10^{2}$, esta transferencia siempre es máxima cerca del fondo de la cavidad, aunque este máximo toma valores más grandes mientras mayor sea el tiempo $t$ de evolución considerado, y posteriormente disminuye con la altura; en tiempos iniciales, $T=.0025$ y $T=.005$, las respectivas curvas en la primera mitad de la frontera parecen fundirse una con otra, mientras que en la segunda mitad se diferencian claramente; para los tiempos finales considerados, $T=.04$ y $T=.08$, las curvas respectivas son casi idénticas y no se distinguen fácilmente una de la otra. 


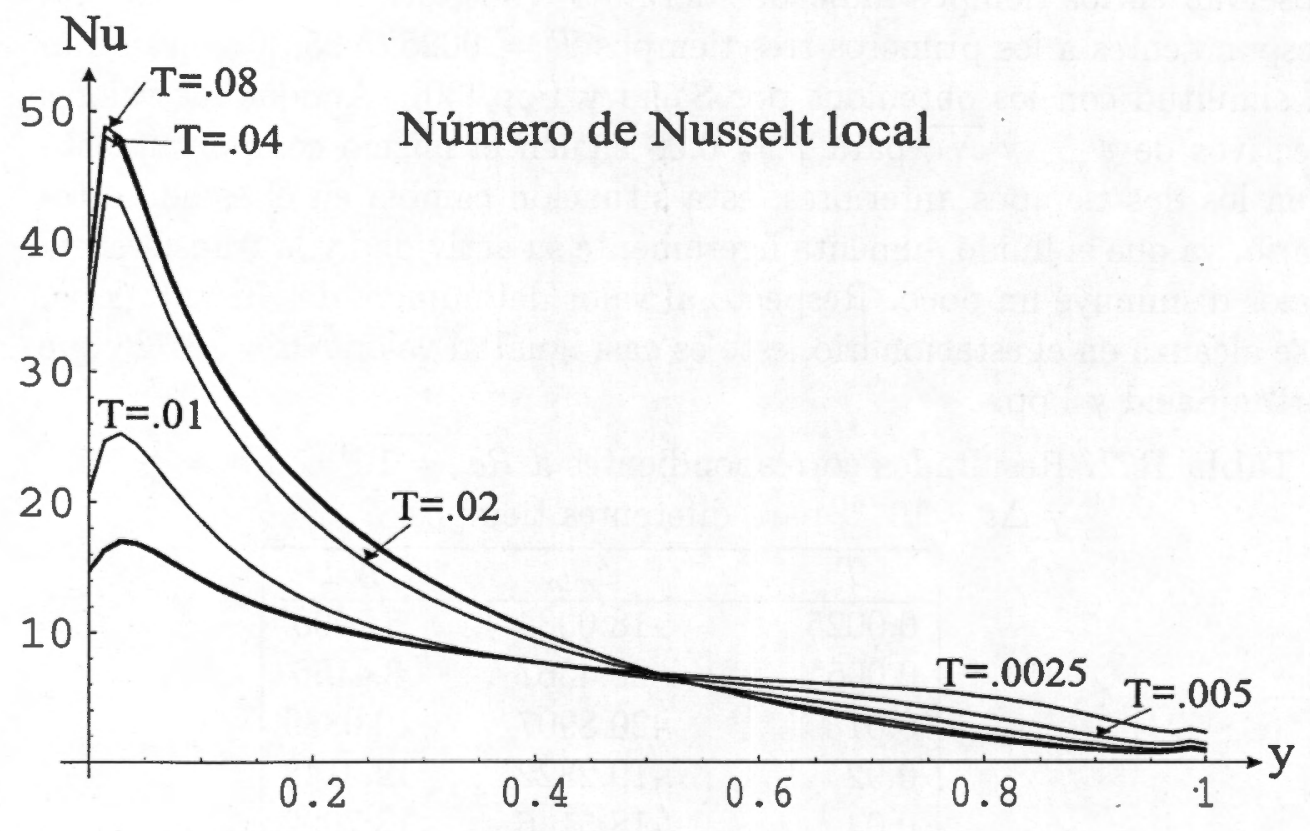

Fig. 3.34: $R a_{p}=10^{3}, h=\frac{1}{70}, \Delta t=10^{-6}$ 


\section{Capítulo 4}

\section{Esquemas numéricos en variables secundarias}

En este capítulo se transforman los correspondientes sistemas de ecuaciones adimensionales que describen el flujo de fluidos por convección natural en medios libres y en medios porosos, descritos en el capítulo 3, en términos de la función de corriente $\psi$ y de la vorticidad $\omega$, que se conocen como variables secundarias, además de la temperatura $\theta$. Se señalan las dificultades e inconvenientes que se presentan al trabajar con estas nuevas variables, posteriormente se describe el procedimiento numérico empleado para resolver los sistemas resultantes, así como una forma de determinar la condición de frontera adecuada para la vorticidad $\omega$; finalmente se muestran los resultados obtenidos al aplicar el esquema a regiones bidimensionales rectangulares para diferentes valores de los parámetros involucrados: número de Rayleigh, $R a_{l}$ - $R a_{p}$, la razón geométrica $A$, y el ángulo de inclinación $\phi$ (el cual no es considerado en variables primitivas).

\subsection{Ecuaciones adimensionales en variables secundarias}

A partir de las ecuaciones adimensionales respectivas, una simplificación adicional a la estructura incompresible que se obtiene, como consecuencia de la aproximación de Boussinesq, es la simplificación en términos de las variables función de corriente y vorticidad en regiones de flujo bidimensional.

Para lograr esto, observamos que por medio de la ecuación de continuidad 
se pueden relacionar las componentes de la velocidad y además, para el caso especial del flujo bidimensional de un fluido incompresible, permite expresar una de las componentes de la velocidad en términos de la otra. En este caso, las dos componentes de la velocidad pueden describirse en términos de la misma función escalar $\psi$, llamada función de corriente, como

$$
u_{1}=\frac{\partial \psi}{\partial y}, \quad u_{2}=-\frac{\partial \psi}{\partial x}
$$

donde $\mathbf{u}=\left(u_{1}, u_{2}\right)$.

Así, de estas expresiones puede verificarse fácilmente que $\psi$ satisface la ecuación de continuidad. Además, podemos considerar la rotación del fluido en un punto, para ello hacemos uso de la vorticidad $\omega$ que, para un flujo bidimensional, se define como $\omega \mathbf{k}=\nabla \times \mathbf{u}$, de donde se tiene entonces que $\omega=\frac{\partial u_{2}}{\partial x}-\frac{\partial u_{1}}{\partial y}$. En este contexto, y para el caso de flujos en medios libres, cuando aplicamos el rotacional a la ecuación de momento, ecuación (2.11b), obtenemos la siguiente ecuación para la vorticidad

$$
\omega_{t}-\frac{1}{R e} \nabla^{2} \omega+\mathbf{u} \cdot \nabla \omega=\frac{R a_{l}}{P r R e^{2}} \frac{\partial \theta}{\partial x}
$$

Finalmente, de acuerdo a la definición de $\omega$ y tomando en cuenta que las componentes $u_{1}$ y $u_{2}$ se expresan en términos de la función de corriente $\psi$, es posible deducir la siguiente ecuación diferencial para $\psi$

$$
-\omega=\frac{\partial^{2} \psi}{\partial x^{2}}+\frac{\partial^{2} \psi}{\partial y^{2}}=\nabla^{2} \psi
$$

De esta forma y para el caso bidimensional, en medios libres se obtiene el siguiente conjunto de ecuaciones escalares, en términos de las variables función de corriente y vorticidad, el cual es equivalente al sistema (2.11).

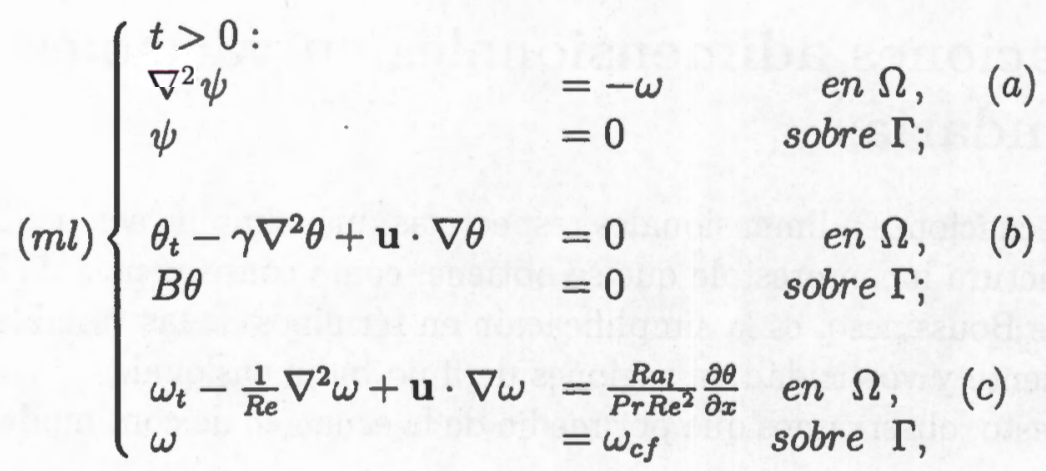


donde $\gamma=\frac{1}{\operatorname{RePr}}$.

La condición de frontera para la vorticidad, desconocida hasta el momento, se indica por $\omega_{c f}, \mathrm{y}$ además, la vorticidad requiere también de una condición inicial $\omega(\mathbf{x}, 0)=\omega(\mathbf{x})$ la cual debe ser igual a $\frac{\partial u_{20}}{\partial x}-\frac{\partial u_{10}}{\partial y}$.

$\mathrm{Si}$ el problema en medios libres es descrito originalmente por el sistema alterno (2.13), entonces al reformularlo en términos de las variables secundarias, función de corriente y vorticidad, adquiere la siguiente forma

$$
(m l)\left\{\begin{array}{llrl}
t>0: & \multicolumn{1}{c}{\text { en } \Omega, \quad(a)} \\
\nabla^{2} \psi & =-\omega & \text { sobre } \Gamma ; \\
\psi & =0 & \\
\theta_{t}-\frac{1}{\sqrt{R a_{l} P r}} \nabla^{2} \theta+\mathbf{u} \cdot \nabla \theta & =0 & \text { en } \Omega, \quad(b) \\
B \theta & =0 & \text { sobre } \Gamma ; \\
\omega_{t}-\sqrt{\frac{P r}{R a_{l}}} \nabla^{2} \omega+\mathbf{u} \cdot \nabla \omega & =\frac{\partial \theta}{\partial x} & \text { en } \Omega, \quad(c) \\
\omega & =\omega_{c f} & \text { sobre } \Gamma,
\end{array}\right.
$$

En el caso de medios porosos, sólo se obtiene la ecuación diferencial que gobierna $\psi$, pues debido a la forma que presenta la respectiva ecuación de momento, ecuación (2.12b), cuando se aplica a ésta el rotacional, en el lado izquierdo de la ecuación resultante únicamente aparece el negativo de la vorticidad, por lo que en virtud de la relación dada por (4.3) se obtiene que

$$
\nabla^{2} \psi=-R a_{p} \frac{\partial \theta}{\partial x}
$$

Por esta razón, el sistema de ecuaciones en medios porosos, a diferencia del que se obtuvo para medios libres, consta de sólo dos ecuaciones, las cuales se sintetizan a continuación

$$
(m p)\left\{\begin{array}{llrl}
t>0: & & \\
\nabla^{2} \psi & =-R a_{p} \frac{\partial \theta}{\partial x} & \text { en } \Omega, \quad(a) \\
\psi & =0 & \text { sobre } \Gamma ; \\
\theta_{t}-\nabla^{2} \theta+\mathbf{u} \cdot \nabla \theta & =0 & \text { en } \Omega, \quad(b) \\
B \theta & =0 & \text { sobre } \Gamma
\end{array}\right.
$$

y como consecuencia, al no estar presente la vorticidad, solamente hay que tratar con las condiciones de frontera para $\psi$ y $\theta$, esta última dada por el operador B, que involucra una condición de frontera de tipo mixta. 
Por último, señalamos que si la región donde se desarrolla el flujo es una cavidad bidimensional inclinada, que se obtiene al rotarla un ángulo $\phi$ en el sentido contrario al giro de las manecillas del reloj, esto significa que la fuerza gravitacional tiene componentes distintas de cero (en el caso sin inclinación, $\phi=0^{\circ}$, sólo la componente vertical es no nula) y, en tal caso, el vector unitario e está dado por $\mathrm{e}=(-\operatorname{sen} \phi,-\cos \phi)$, con lo cual las ecuaciones dadas por (4.4c) y (4.7a) se ven modificadas y están dadas respectivamente por

$$
\begin{aligned}
& \omega_{t}-\frac{1}{R e} \nabla^{2} \omega+\mathbf{u} \cdot \nabla \omega=\frac{R a_{l}}{P r R e^{2}}\left(\cos \phi \frac{\partial \theta}{\partial x}-\operatorname{sen} \phi \frac{\partial \theta}{\partial y}\right) \quad \text { en } \Omega \\
& \nabla^{2} \psi=-R a_{p}\left(\cos \phi \frac{\partial \theta}{\partial x}-\operatorname{sen} \phi \frac{\partial \theta}{\partial y}\right) \quad \text { en } \Omega
\end{aligned}
$$

De esta manera los sistemas respectivos, tanto en medios libres como en medios porosos, en cavidades inclinadas son

$$
\begin{aligned}
& (m l)\left\{\begin{array}{llr}
t>0: & & \\
\nabla^{2} \psi & =-\omega & \text { en } \Omega, \quad(a) \\
\psi & \text { sobre } \Gamma \\
\theta_{t}-\gamma \nabla^{2} \theta+\mathbf{u} \cdot \nabla \theta & =0 & \text { en } \Omega, \quad(b) \\
B \theta & =0 & \text { sobre } \Gamma ; \\
& & \\
\omega_{t}-\frac{1}{R e} \nabla^{2} \omega+\mathbf{u} \cdot \nabla \omega & =\frac{R a_{l}}{P r R e^{2}}\left(\cos \phi \frac{\partial \theta}{\partial x}-\operatorname{sen} \phi \frac{\partial \theta}{\partial y}\right) & \text { en } \Omega, \quad(c) \\
\omega & =\omega_{c f} & \text { sobre } \Gamma,
\end{array}\right. \\
& (m p)\left\{\begin{array}{llr}
t>0: & =-R a_{p}\left(\cos \phi \frac{\partial \theta}{\partial x}-\operatorname{sen} \phi \frac{\partial \theta}{\partial y}\right) & \text { en } \Omega, \quad(a) \\
\nabla^{2} \psi & \text { sobre } \Gamma ; \\
\psi & =0 & \text { en } \Omega, \quad(b) \\
\theta_{t}-\nabla^{2} \theta+\mathbf{u} \cdot \nabla \theta & =0 & \text { sobre } \Gamma \\
B \theta & =0
\end{array}\right.
\end{aligned}
$$

Los sistemas (4.8) y (4.9) representan la aproximación de Boussinesq en variables función de corriente y vorticidad en medios libres y en medios porosos respectivamente, en cavidades que tienen un ángulo de inclinación $\phi$. En el caso del sistema alterno (4.5), el lado derecho de la ecuación (4.5c) cambia por $\left(\cos \phi \frac{\partial \theta}{\partial x}-\operatorname{sen} \phi \frac{\partial \theta}{\partial y}\right)$ en cavidades inclinadas. Como puede observarse, 
en cualquiera de los sistemas obtenidos, el término asociado a la presión $p$ desaparece.

Respecto a las dificultades que involucra esta nueva formulación de los problemas, podemos mencionar: 1) el acoplamiento entre las ecuaciones correspondientes, para ambos medios, 2) la no linealidad presente en las ecuaciones de energía y vorticidad, en medios libres, debido a los términos convectivos; en medios porosos, esta no linealidad sólo se presenta en la ecuación de energía, 3) no se cuenta con una condición de frontera para la ecuación de la vorticidad.

Con el objeto de preservar el acoplamiento natural de los fenómenos, aplicamos una discretización en tiempo en forma implícita, con lo cual todas las variables se consideran en el nivel de tiempo que se quiere conocer, incluyendo las que aparecen en las ecuaciones que no dependen explícitamente del tiempo. Con esta discretización temporal se obtiene un sistema nolineal de ecuaciones elípticas. Para resolver este sistema, la no-linealidad y el acoplamiento entre las ecuaciones deben manejarse apropiadamente, lo cual puede lograrse mediante un proceso iterativo que conduzca a resolver en cada iteración problemas elípticos lineales, simétricos y desacoplados.

\subsection{Esquema numérico en medios libres}

Considerando que el problema semidiscreto en tiempo se obtiene también haciendo uso de la aproximación de segundo orden dada por (3.1) para las correspondientes derivadas temporales, iniciamos el proceso indicando una forma de obtener la condición de frontera que se requiere para la vorticidad. Posteriormente se describe el método iterativo de punto fijo para resolver el sistema de ecuaciones correspondiente. Terminamos presentando resultados para flujos de convección natural en regiones rectangulares inclinadas.

En el caso de medios libres, se requiere de una forma adecuada para determinar la condición de frontera para la vorticidad $\omega$. Podemos señalar que el hecho de que la condición de incompresibilidad se satisfaga automáticamente se puede ver como una ventaja en contra de la desventaja de no tener condición de frontera para la vorticidad; por otro lado, en la frontera $\Gamma, \psi$ está sobredeterminada, Peyret y Taylor [52] y Dean et al. [53], pues además de conocerse $\left.\psi\right|_{\Gamma}$ también se conoce $\left.\frac{\partial \psi}{\partial n}\right|_{\Gamma}$.

Ya que por un lado, en fluidos viscosos la velocidad es cero en paredes sólidas y fijas, y por otro lado, por (4.1), $\psi$ es constante, podemos consi- 
derar esta constante como cero sobre la frontera $\Gamma$. Una forma de obtener la condición de frontera para $\omega$, es realizar una expansión de Taylor de $\psi$ en la frontera usando la relación (4.4a), y considerando la adaptación a convección natural de la de convección mixta que se usa en Nicolás y Bermúdez [12]. Así, obtenemos las siguientes relaciones para la condición de frontera $\omega_{c f}$, dadas implícitamente por valores de $\psi$ (no conocidos todavía) en el interior,

$$
\begin{array}{ll}
\omega(0, y, t)=\frac{1}{2 h_{x}^{2}}\left[8 \psi\left(h_{x}, y, t\right)-\psi\left(2 h_{x}, y, t\right)\right] & +O\left(h_{x}^{2}\right) \\
\omega(a, y, t)=\frac{1}{2 h_{x}^{2}}\left[8 \psi\left(a-h_{x}, y, t\right)-\psi\left(a-2 h_{x}, y, t\right)\right] & +O\left(h_{x}^{2}\right) \\
\omega(x, 0, t)=\frac{1}{2 h_{y}^{2}}\left[8 \psi\left(x, h_{y}, t\right)-\psi\left(x, 2 h_{y}, t\right)\right] & +O\left(h_{y}^{2}\right) \\
\omega(x, b, t)=\frac{1}{2 h_{y}^{2}}\left[8 \psi\left(x, b-h_{y}, t\right)-\psi\left(x, b-2 h_{y}, t\right)\right] & +O\left(h_{y}^{2}\right)
\end{array}
$$

donde $h_{x}$ y $h_{y}$ denotan los pasos de discretización espacial en las direcciones $\mathrm{X}$ y $\mathrm{Y}$ respectivamente.

Una vez que hemos resuelto el problema de no contar con una condición de frontera para $\omega$, procedemos, a continuación, a describir el esquema numérico correspondiente.

Aplicamos la aproximación de segundo orden dada por (3.1) en las derivadas temporales $\theta_{t}$ y $\omega_{t}$ que aparecen en el sistema (4.8), por lo que el correspondiente sistema semidiscreto que se obtiene es

$$
\begin{aligned}
& \nabla^{2} \psi^{n+1}=-\omega^{n+1} \\
& \psi^{n+1}=0 \\
& \alpha \theta^{n+1}-\gamma \nabla^{2} \theta^{n+1}+\mathbf{u}^{\mathbf{n}+1} \cdot \nabla \theta^{n+1}=f_{\theta} \quad \text { en } \Omega \text {, } \\
& B \theta^{n+1}=0 \\
& \begin{array}{l}
\text { en } \Omega \text {, } \\
\text { sobre } \Gamma \text {; }
\end{array} \\
& \alpha \omega^{n+1}-\frac{1}{R e} \nabla^{2} \omega^{n+1}+\mathbf{u}^{n+1} \cdot \nabla \omega^{n+1}=\frac{R a l}{P r R e^{2}}\left(\cos \phi{\frac{\partial \theta^{n+1}}{\partial x}}^{n+1}\right. \\
& \omega^{n+1}=\omega_{c f}
\end{aligned}
$$

donde $n \geq 1, \alpha=\frac{3}{2 \Delta t}, f_{\theta}=\frac{4 \theta^{n}-\theta^{n-1}}{2 \Delta t}, f_{\omega}=\frac{4 \omega^{n}-\omega^{n-1}}{2 \Delta t}$. El término $\omega_{c f}$ denota la condición de frontera para la vorticidad dada por (4.10) y $\mathbf{u}=\left(u_{1}, u_{2}\right)$ está dada por (4.1) en términos de $\psi$.

Si renombramos $\left(\psi^{n+1}, \theta^{n+1}, \omega^{n+1}\right)$ como $(\psi, \theta, \omega)$ entonces en cada nivel 
de tiempo debemos resolver el sistema de ecuaciones elípticas no lineal

$$
\begin{array}{lr}
\nabla^{2} \psi=-\omega & \text { en } \Omega, \\
\psi=0 & \text { sobre } \Gamma \\
\alpha \theta-\gamma \nabla^{2} \theta+\mathbf{u} \cdot \nabla \theta=f_{\theta} & \text { en } \Omega, \\
B \theta=0 & \text { sobre } \Gamma \\
\alpha \omega-\frac{1}{R e} \nabla^{2} \omega+\mathbf{u} \cdot \nabla \omega=\frac{R a l}{P r R e^{2}}\left(\cos \phi \frac{\partial \theta}{\partial x}-\operatorname{sen} \phi \frac{\partial \theta}{\partial y}\right) & \\
+f_{\omega} & \text { en } \Omega, \\
\omega=\omega_{c f} & \text { sobre } \Gamma
\end{array}
$$

La forma en que resolvemos el sistema (4.12) en cada nivel de tiempo $(n+1) \Delta t$, es mediante un método iterativo de punto fijo, para lo cual usamos la notación

$$
\Theta(\theta, \psi) \equiv \alpha \theta-\gamma \nabla^{2} \theta+\left(u_{1}, u_{2}\right) \cdot \nabla \theta-f_{\theta}
$$

y

$$
\begin{aligned}
R(\omega, \psi) \equiv & \alpha \omega-\frac{1}{R e} \nabla^{2} \omega+\left(u_{1}, u_{2}\right) \cdot \nabla \omega \\
& -\frac{R a_{l}}{P r R e^{2}}\left(\cos \phi \frac{\partial \theta}{\partial x}-\operatorname{sen} \phi \frac{\partial \theta}{\partial y}\right)-f_{\omega}
\end{aligned}
$$

Entonces, el sistema (4.12), para medios libres, es equivalente a

$$
\begin{array}{llll}
\nabla^{2} \psi=-\omega & \text { en } \Omega, \psi=0 & \text { sobre } \Gamma, \\
\Theta(\theta, \psi)=0 & \text { en } \Omega, B \theta=0 & \text { sobre } \Gamma \\
R(\omega, \psi)=0 & \text { en } \Omega, \omega=\omega_{\text {cf }} & \text { sobre } \Gamma
\end{array}
$$

luego, el sistema (4.15) lo resolvemos mediante el siguiente proceso iterativo de punto fijo.

Con $\left(\psi^{o}, \theta^{o}, \omega^{o}\right)=\left(\psi^{n}, \theta^{n}, \omega^{n}\right)$ conocidos, resolvemos hasta obtener convergencia

$$
\begin{array}{lr}
\nabla^{2} \psi^{m+1}=-\omega^{m} & \text { en } \Omega, \\
\psi=0 & \text { sobre } \Gamma, \\
\theta^{m+1}=\theta^{m}-\lambda\left(\alpha-\gamma \nabla^{2}\right)^{-1} \Theta\left(\theta^{m}, \psi^{m+1}\right) & \text { en } \Omega, \\
B \theta=0 & \text { sobre } \Gamma, \\
\omega^{m+1}=\omega^{m}-\lambda\left(\alpha-\frac{1}{R e} \nabla^{2}\right)^{-1} R\left(\omega^{m}, \psi^{m+1}\right) & \text { en } \Omega, \\
\omega=\omega_{c f} & \text { sobre } \Gamma,
\end{array}
$$


y tomamos $\left(\theta^{n+1}, \omega^{n+1}, \psi^{n+1}\right)=\left(\theta^{m+1}, \omega^{m+1}, \psi^{m+1}\right)$. Respecto a los valores $\left(\psi^{1}, \theta^{1}, \omega^{1}\right)$ que se requieren dentro del proceso, a través de $f_{\theta}$ y $f_{\omega}$, se obtienen de manera similar a lo indicado en el caso de variables primitivas.

Un aspecto distintivo del esquema numérico en medios libres, es que el método iterativo debe extenderse hasta la frontera para poder construir la condición de frontera de la vorticidad, dada en términos de valores de $\psi$, todavía desconocidos, en el interior de la región.

Por último, hacemos la observación de que la segunda ecuación del sistema (4.16) es equivalente a

$$
\left(\alpha-\gamma \nabla^{2}\right) \theta^{m+1}=\left(\alpha-\gamma \nabla^{2}\right) \theta^{m}-\lambda \theta\left(\theta^{m}, \psi^{m+1}\right)
$$

mientras que la tercera es equivalente a

$$
\left(\alpha-\frac{1}{R e} \nabla^{2}\right) \omega^{m+1}=\left(\alpha-\frac{1}{R e} \nabla^{2}\right) \omega^{m}-\lambda R\left(\omega^{m}, \psi^{m+1}\right)
$$

De esta manera, la solución del problema involucra resolver $3(m+1)$ problemas elípticos lineales y simétricos en cada en cada nivel de tiempo, para lo cual nuevamente empleamos el paquete Fishpack, con la opción de segundo orden; por otro lado, tomando en cuenta que todas las primeras derivadas espaciales de $\psi, \theta$ y $\omega$ se aproximan mediante diferencias finitas de segundo orden adecuadas, esto es, centradas, regresivas o progresivas (usando (3.1)), se concluye que el esquema numérico se basa en aproximaciones de segundo orden.

\subsubsection{Resultados numéricos}

Los experimentos numéricos se desarrollan en cavidades rectangulares, donde la condición de frontera para la temperatura nuevamente está dada por

$$
\left.\frac{\partial \theta}{\partial n}\right|_{y=0, b}=\left.0 \quad \theta\right|_{x=0}=\left.0.5 \quad \theta\right|_{x=a}=-0.5
$$

y las respectivas condiciones iniciales son: $\theta(\mathbf{x}, 0)=0, \psi(\mathbf{x}, 0)=0 \quad$ y $\omega(\mathbf{x}, 0)=0$. Los resultados que presentamos en esta sección conciernen a flujos de estado estacionario, donde $\operatorname{Pr}=0.71,10^{4} \leq R a_{l} \leq 10^{6} \mathrm{y}$ $0^{\circ} \leq \phi \leq 360^{\circ}$. En el proceso iterativo se considera que $\lambda=0.7$, y para determinar $T_{\text {est }}$ empleamos el criterio de convergencia dado por (3.9) con tolerancia $10^{-5}$, aplicado a la función de corriente $\psi$ y a la temperatura $\theta$. Los resultados que se muestran en la primera subsección corresponden a flujos 
en la cavidad cuadrado unitaria, $A=1$, mientras que en la segunda se presentan los que se obtienen en una cavidad rectangular con razón geométrica $A=8$. Algunos de los experimentos numéricos tienen como objetivo validar el esquema numérico, al compararlos con resultados conocidos y que se supone son correctos; otros se consideran resultados nuevos, los cuales se obtuvieron mediante estudios de independencia de malla y de paso de tiempo considerando el error relativo discreto $L_{\infty}$ en $\bar{\Omega}$

$$
\begin{gathered}
\text { con } \Delta t \text { fijo: } \frac{\left\|f_{h x_{1}, h h_{1} ; \Delta t}-f_{h x_{2}, h y_{2} ; \Delta t}\right\|_{\infty}}{\left\|f_{h x_{1}, h y_{1} ; \Delta t}\right\|_{\infty}} \\
\text { con } h_{x} \times h_{y} \text { fija: } \frac{\left\|f_{h_{x}, h_{y} ; \Delta t_{1}}-f_{h x, h y_{i} \Delta t_{2}}\right\|_{\infty}}{\left\|f_{h x, h y ;} ; t_{1}\right\|_{\infty}},
\end{gathered}
$$

donde $h_{x}$ y $h_{y}\left(h\right.$ si $h_{x}=h_{y}$ ) representan, nuevamente, el tamaño de la malla en las direcciones $\mathrm{X}$ y $\mathrm{Y}, \mathrm{y} \Delta t$ el paso de tiempo.

1) Cavidad cuadrada inclinada $(a=1=b)$

Los experimentos numéricos se llevan a cabo considerando el sistema (4.8), para lo cual se emplea una malla uniforme con $h=\frac{1}{30}$ y $\Delta t=10^{-4}$ independientemente del valor del Número de Rayleigh $R a_{l}$ empleado. En el caso de $R a_{l} \leq 10^{3}$ los resultados muestran que las isotermas son casi paralelas a las paredes laterales y las líneas de corriente se ordenan en una sola celda en el centro de la cavidad, signo característico de sistemas donde la conducción de calor es el mecanismo predominante; además, se observa poca variación del número de Nusselt global $\overline{\mathrm{Nu}}$, así como del valor mínimo que alcanza la función de corriente $\psi_{\min }$, independientemente del ángulo de inclinación de la cavidad. Para $R a_{l}=10^{4}$, aunque las isotermas empiezan a mostrar algunos cambios y ya no son paralelas a las fronteras laterales, las líneas de corriente siguen mostrando una sola celda central. Los resultados correspondientes a estos dos valores de $R a_{l}$ no se muestran en el presente trabajo.

En la Figura 4.1 se muestran los resultados obtenidos para $R a_{l}=10^{5}$ con diversos ángulos de inclinación. En este caso, el movimiento del fluido es mayor en comparación con aquellos que se comentaron para $R a_{l}$ menores. Los resultados correspondientes a $\phi=0^{\circ}$, son los mismos que se muestran en la Figura 3.1 para el caso de variables primitivas, y que ya se comentaron; al girar la cavidad a partir de este ángulo, en dirección positiva respecto a la horizontal, las isotermas muestran mayor o menor deformación en el centro de la cavidad, mientras que las líneas de corriente muestran una sola 


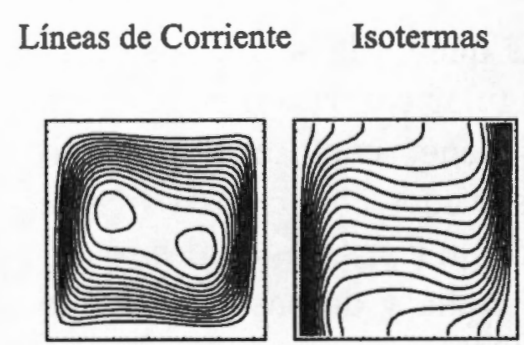

$0^{\circ}$

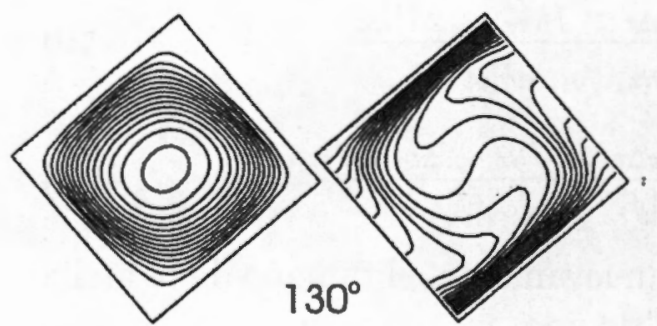

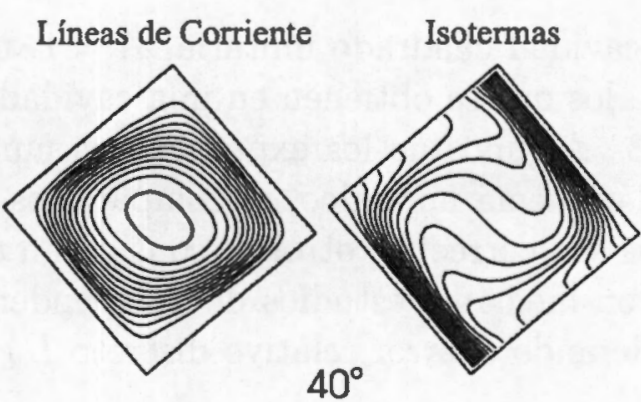

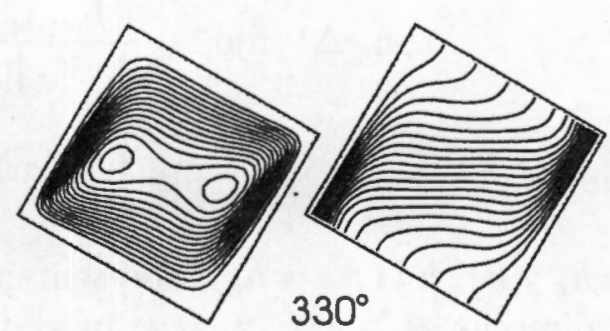

$\mathrm{Nu}$ Número de Nusselt local

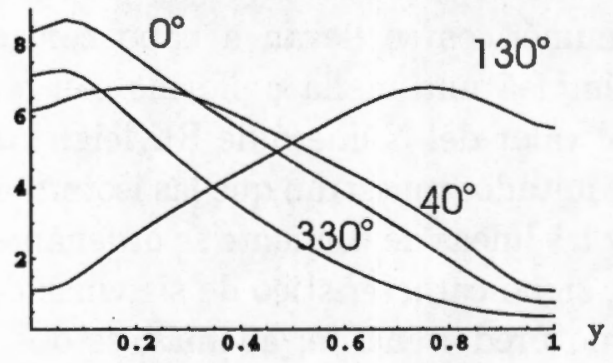

Fig. 4.1: $R a_{l}=10^{5}, A=1, h=\frac{1}{30}$ y $\Delta t=10^{-4}$.

celda principal, en donde la parte central de éstas tienden a redondearse o a alargarse, dependiendo de la inclinación de la cavidad, e incluso en algunos ángulos aparecen dos celdas secundarias. Para los ángulos $0^{\circ}, 40^{\circ}$ y $330^{\circ}$ la celda principal rota en dirección de las manecillas del reloj, mientras que para $130^{\circ}$ gira en sentido opuesto. Por otra parte, y tomando como referencia la cavidad sin inclinación, la gráfica del número de Nusselt local sobre la frontera caliente indica que el máximo se alcanza cerca de la esquina inferior para los ángulos $0^{\circ}, 40^{\circ}$ o $330^{\circ}$, y cerca de la superior para $130^{\circ}$; en los primeros tres ángulos se observa que la curva correspondiente decrece suavemente desde la esquina inferior hacia la superior, y esta situación se presenta de manera opuesta cuando el ángulo es de $130^{\circ}$. 
Tabla L.1. Resultados para $R a_{l}=10^{5}$, con $A=1$,

$h=\frac{1}{30} . y \Delta t=10^{-4}$.
\begin{tabular}{|c|c|c|c|}
\hline$\phi$ & $\psi_{\min / \max }$ & $\overline{N u}$ & $T_{\text {est }}$ \\
\hline $0^{\circ}$ & -13.4661 & 4.7504 & 0.2175 \\
$40^{\circ}$ & -24.6617 & 4.7386 & 0.2426 \\
$130^{\circ}$ & 28.7379 & 4.6638 & 0.2843 \\
$330^{\circ}$ & -7.2228 & 3.1358 & 0.2386 \\
\hline
\end{tabular}

En la Tabla L.1 se indican los resultados que se obtienen para el número de Nusselt global $\overline{N u}$, el valor extremo de la función de corriente $\psi_{\text {min/máx }}$, así como el tiempo $T_{\text {est }}$ que se requiere para alcanzar el estado estacionario cuando $R a_{l}=10^{5}$. Se observa que el movimiento del fluido es mayor cuando el ángulo es de $130^{\circ}$, y la transferencia de calor disminuye al incrementar el ángulo.

Con objeto de validar el flujo que se obtiene para $R a_{l}=10^{6}$ con el presente esquema numérico, se realizaron cálculos, tomando como base la cavidad sin inclinación, con tres tamaños de malla así como con tres pasos de tiempo distintos, los cuales se indican a continuación:

(1) paso de tiempo $\Delta t=10^{-4}$ fijo y $h=1 / 30,1 / 45,1 / 60$;

(2) tamaño de malla $h=1 / 30$ fija y $\Delta t=10^{-4}, 5 \times 10^{-5}, 2.5 \times 10^{-5}$.

Las diferencias respectivas para cada conjunto de valores fueron:

(1) a lo más $7.7 \%$ ( $7.7 \%$ para la vorticidad, $2.2 \%$ para la función de corriente y $5.4 \%$ para la temperatura);

(2) menos de $1 \%$ ( $0.14 \%$ para la vorticidad, $0.13 \%$ para la función de corriente y $0.08 \%$ para la temperatura).

El valor máximo de la función de corriente $\psi$ es siempre cero, mientras que el correspondiente valor mínimo en cada caso es:

(1) $\min =-23.8070,-23.6150,-23.6034$,

(2) $\min =-23.8070,-23.8043,-23.7993$.

De esta manera, se considera que el flujo de la Figura 4.2 es correcto debido a lo reducido de las discrepancias mostradas arriba, además de que no se observaron cambios en los resultados al considerar mallas más finas. Como puede apreciarse, el flujo obtenido muestra una estructura más compleja, como lo demuestra el hecho de que para algunos ángulos, en la gráfica correspondiente a las líneas de corriente aparecen dos y hasta tres celdas 


\section{Lineas de Corriente Isotermas}

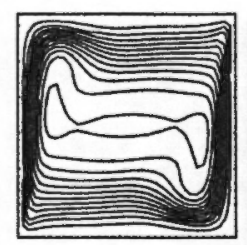

$0^{\circ}$
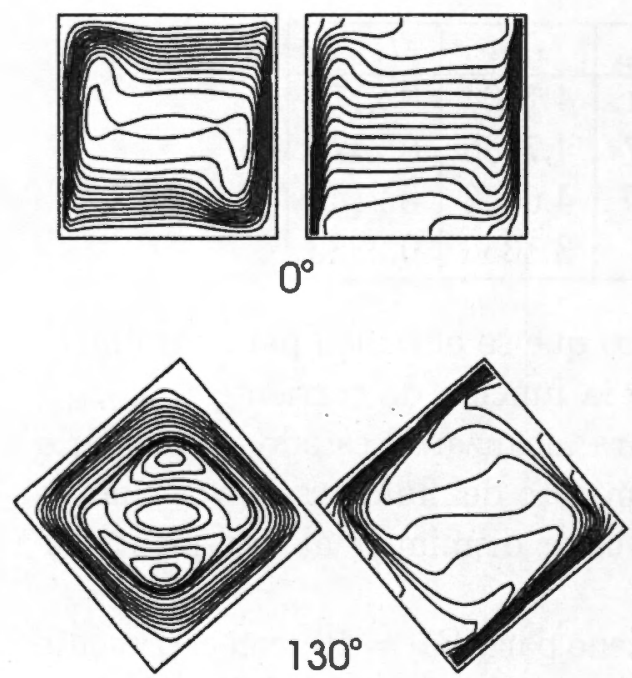

$130^{\circ}$
Lineas de Corriente
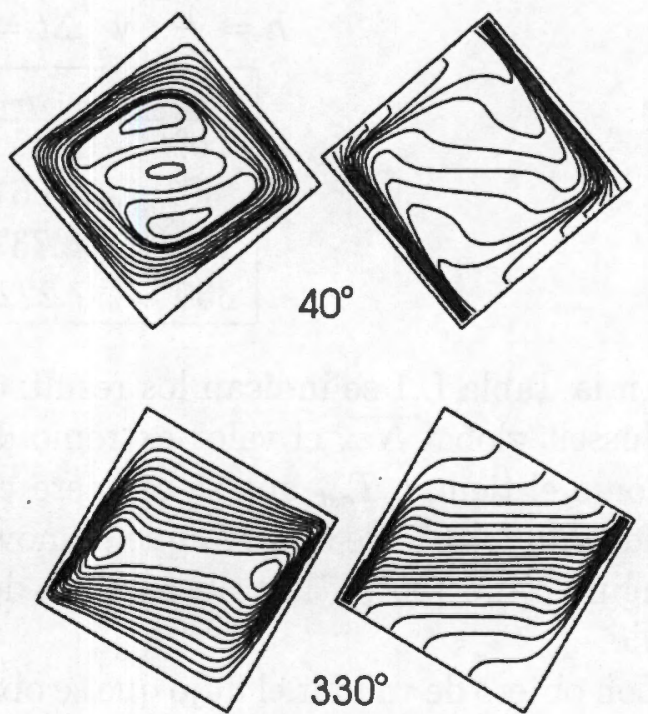

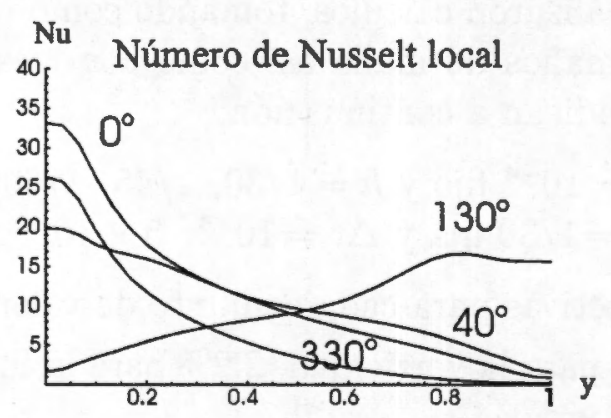

Fig. 4.2: $R a_{l}=10^{6}, A=1, h=\frac{1}{30}$ y $\Delta t=10^{-4}$.

secundarias. La zona central de estas líneas de corriente se expanden hacia todas las paredes de la cavidad, y en algunos ángulos esta expansión es más notoria hacia las paredes que tienen temperatura prescrita, en donde los contornos parecen adherirse a ellas. Respecto a las gráficas del número de Nusselt local, se observa que éstas tienen un descenso más pronunciado que las del caso anterior, cerca de la esquina inferior izquierda, con excepción de la que corresponde a $\phi=130^{\circ}$, donde esta situación es opuesta, esto es, el descenso ocurre cerca de la esquina superior.

Los valores de $\overline{N u}, \psi_{\min / \max }$ y $T_{\text {est }}$ para los cuatro ángulos considerados, en la Tabla L.2, muestran un comportamiento muy similar al observado para el caso de $R a_{l}=10^{5}$; el movimiento más fuerte del fluido se presenta en 
$130^{\circ}$, mientras que la transferencia de calor es máxima en $0^{\circ}$ y disminuye al aumentar el ángulo. Comparando los resultados de las Tablas L.1 y L.2, es claro que el flujo se vuelve más rápido y la transferencia de calor es mayor cuando $R a_{l}$ aumenta.

Tabla L.2. Resultados para $R a_{l}=10^{6}$, con $A=1$, $h=\frac{1}{30}$ y $\Delta t=10^{-4}$.

\begin{tabular}{|c|c|c|c|}
\hline$\phi$ & $\psi_{\min / \operatorname{máx}}$ & $\overline{N u}$ & $T_{\text {est }}$ \\
\hline $0^{\circ}$ & -23.8098 & 11.3083 & .1806 \\
$40^{\circ}$ & -41.8493 & 10.4157 & .2961 \\
$130^{\circ}$ & 47.3883 & 9.9411 & .7188 \\
$330^{\circ}$ & -12.6913 & 6.5755 & .1977 \\
\hline
\end{tabular}

Los resultados obtenidos para $R a_{l}=10^{5}$ y $10^{6}$ con ángulo $\phi=0^{\circ}$ mostraron buena concordancia con los obtenidos por Bermúdez y Nicolás [15], mientras que los obtenidos en la cavidad inclinada para $R a_{l}=10^{6}$, con los ángulos correspondientes, concuerdan con los que Kuyper et al. [54] reportan al considerar una cavidad cuadrada que es calentada por abajo.

Tabla L.3. Número de Nusselt global y valor máximo de las velocidades horizontal y vertical para $\phi=0^{\circ}$ y $\Delta t=10^{-4}$.

\begin{tabular}{|c|c|c|c|c|c|c|}
\hline$R a_{l}$ & $u_{1 \max }(\mathrm{M})$ & $u_{2 \max }(\mathrm{M})$ & $\overline{N u}(\mathrm{M})$ & $u_{1 \max }(\mathrm{B})$ & $u_{2 \max }(\mathrm{B})$ & $\overline{N u}(\mathrm{~B})$ \\
\hline $10^{3}$ & 3.54 & 3.59 & 1.108 & 5.081 & 5.140 & 1.117 \\
\hline $10^{4}$ & 16.18 & 19.44 & 2.201 & 22.513 & 27.017 & 2.257 \\
\hline $10^{5}$ & 42.51 & 69.08 & 4.430 & 58.773 & 91.990 & 4.750 \\
\hline $10^{6}$ & 117.8 & 226.7 & 8.754 & 172.644 & 295.914 & 9.245 \\
\hline
\end{tabular}

En la Tabla L.3 se muestra una relación comparativa correspondientes al número de Nusselt global $\overline{N u}$, así como a los valores máximos de las velocidades, horizontal y vertical, $u_{1 \max }$ y $u_{2 \max }$ para $10^{3} \leq R a_{l} \leq 10^{6}$, obtenidos en este trabajo (B) y los reportados por Markatos y Pericleous [55], indicados por (M), a partir del problema estacionario. Aunque se observan diferencias importantes, en particular en los valores de las velocidad, los resultados de Markatos y Pericleous se obtuvieron sin considerar la aproximación de Boussinesq, y señalan que los valores correspondientes al número de Nusselt obtenidos para $R a_{l}=10^{5}$ y $10^{6}$ muestran diferencias de hasta $3.7 \%$ con respecto a aquellos que se obtienen cuando esta aproximación si es considerada. 
2) Cavidad rectangular con razón geométrica $A=8$

En este caso, los cálculos se realizan empleando el equivalente al sistema alterno (4.5) para cavidades con inclinación. El flujo se desarrolla en una región rectangular, donde $a=1$ y $b=8$, para valores de $R a_{l}=3.4 \times 10^{5}$ y $10^{6}$; en ambos casos, se emplea una malla de $h_{x} \times h_{y}=1 / 30 \times 8 / 240$, y un paso de tiempo de $\Delta t=10^{-2}$. Las respectivas condiciones de frontera $\mathrm{e}$ iniciales para cada una de las variables son las mismas que se indican al inicio de esta sección. En la primera subsección presentamos resultados para ambos valores de $R a_{l}$ en la cavidad sin inclinación, mientras que en la segunda se muestran los correspondientes a $R a_{l}=3.4 \times 10^{5}$ en la cavidad inclinada.

\section{1) Cavidad sin inclinación}

En la Figura 4.3 se muestran resultados para $R a_{l}=3.4 \times 10^{5}$ en tres tiempos distintos; de manera similar, la Figura 4.4 muestra los correspondientes resultados para $R a_{l}=10^{6}$. En ambas figuras puede observarse que en las líneas de corriente se obtiene una sola celda principal, que gira en dirección negativa, y ésta a su vez contiene varias celdas secundarias, más numerosas para $R a_{l}=10^{6}$; de igual manera, las isotermas muestran algunas distorsiones para este último valor de $R a_{l}$, que se incrementan cerca de la tapa y del fondo de la cavidad. Las respectivas gráficas del número de Nusselt local para $R a_{l}=3.4 \times 10^{5}$, en los distintos tiempos, muestran poca diferencia entre ellos; se observan pequeñas variaciones en las regiones donde se localizan las celdas secundarias, cerca de la parte inferior y hacia la mitad de la pared caliente, éstas últimas se incrementan hacia la parte superior de la misma. Puede observarse que para $R a_{l}=10^{6}$ la transferencia de calor local es mayor, sin embargo ésta presenta varios cambios súbitos importantes, en los tres tiempos indicados, principalmente hacia la parte final de la pared caliente, en donde existe mayor cantidad de celdas secundarias cerca de ésta.

Para ambos valores de $R a_{l}$, se observó el desarrollo de los flujos en tiempos más grandes que los que reportamos en este trabajo y ellos indican que los flujos respectivos no alcanzan el estado estacionario, sino por el contrario son flujos oscilatorios, lo que para el caso de $R a_{l}=3.4 \times 10^{5}$ concuerda con lo que reporta Glowinski [20], en donde se presentan resultados para diversos tiempos $T$ correspondientes a un periodo.

La Tabla L.4 confirma, por un lado, que existe una mayor transferencia de calor sobre la pared izquierda de la cavidad para $R a_{l}=10^{6}$, aunque el va- 

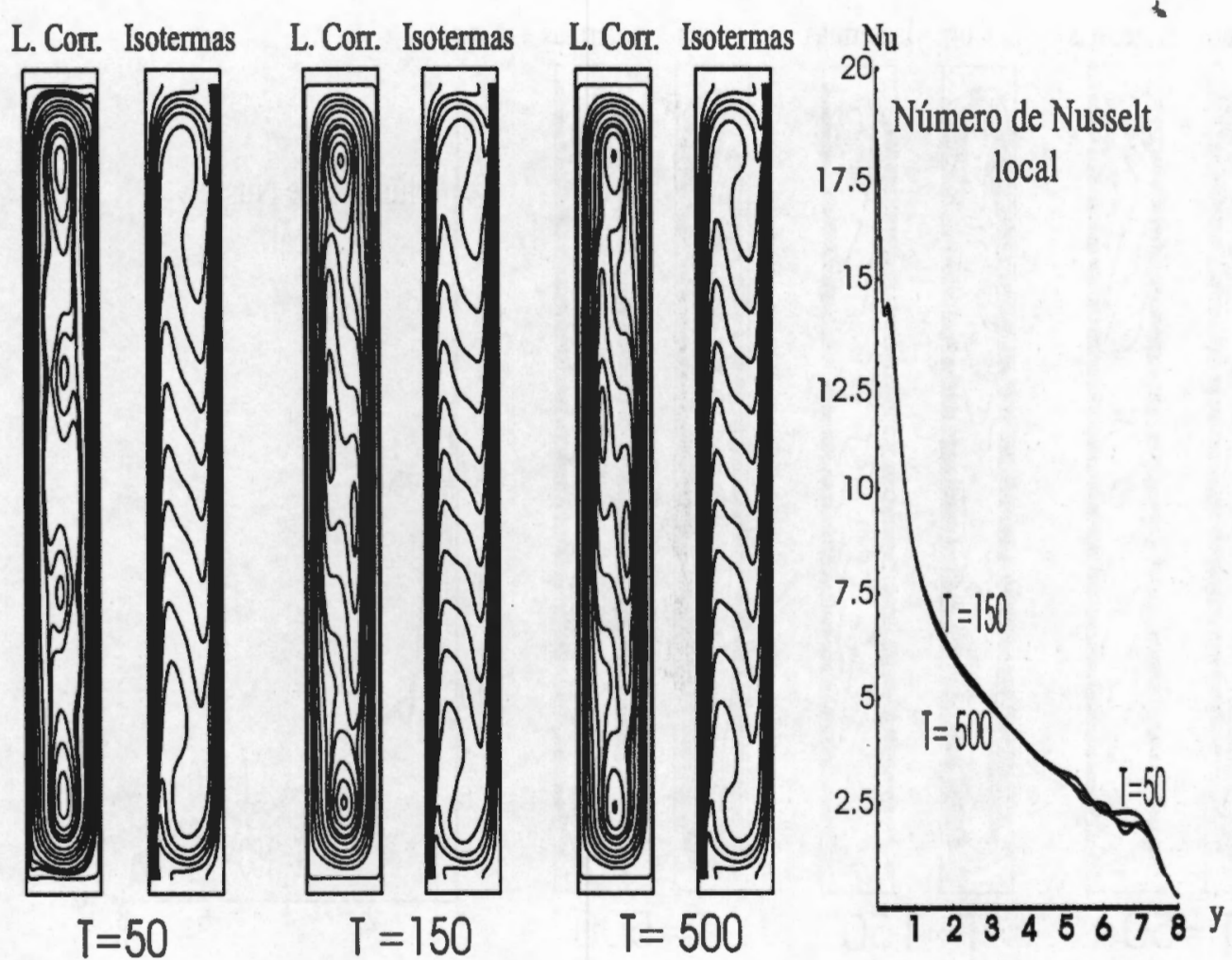

Fig. 4.3: $R a_{l}=3.4 \times 10^{5}, A=8, h_{x} \times h_{y}=\frac{1}{30} \times \frac{8}{240}$ y $\Delta t=10^{-2}$.

Table L.4. Resultados para $R a_{l}=3.4 \times 10^{5}$ y $R a_{l}=10^{6}$ con $A=8$, $h_{x} \times h_{y}=\frac{1}{30} \times \frac{8}{240}$ y $\Delta t=10^{-2}$ (caso sin inclinación).

\begin{tabular}{|c|c|c|c|}
\hline$R a_{l}$ & $\psi_{\min }$ & $\overline{N u}$ & $T$ \\
\hline $3.4 \times 10^{5}$ & -0.1498 & 4.8471 & 50 \\
& -0.1289 & 4.8354 & 150 \\
& -0.1285 & 4.8352 & 500 \\
\hline $10^{6}$ & -0.1737 & 6.6580 & 50 \\
& -0.1097 & 6.5888 & 150 \\
& -0.1207 & 6.6408 & 500 \\
\hline
\end{tabular}

lor de $\psi_{\min }$ indica que el movimiento del fluido, en $T=150$ y $T=500$, es más fuerte para $R a_{l}=3.4 \times 10^{5}$ que para $R a_{l}=10^{6} \mathrm{y}$, por otra parte, con $R a_{l}$ fijo, se observa que la transferencia de calor global $\overline{N u}$ se incrementa cuando el movimiento del fluido aumenta. 


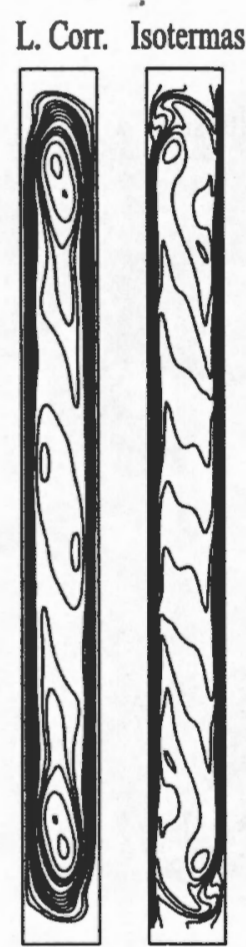

$\mathrm{T}=50$
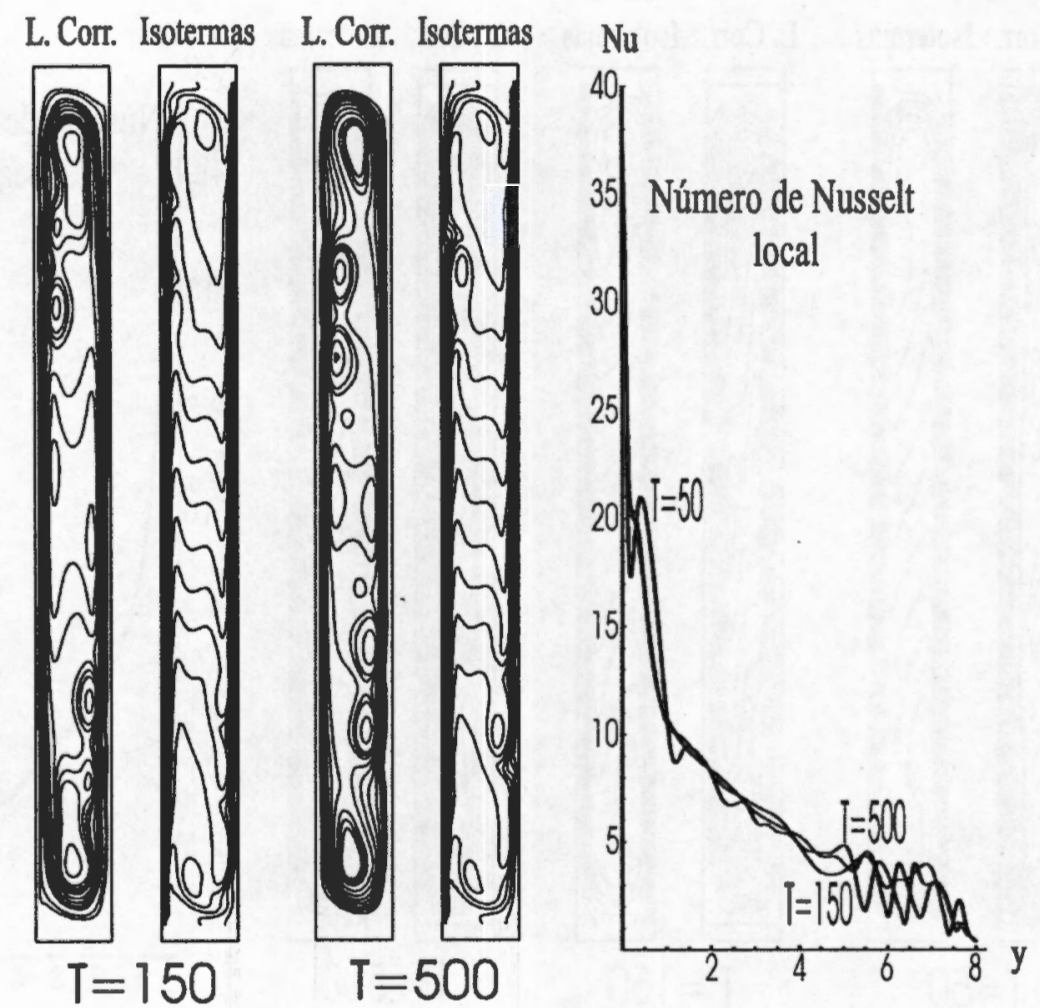

Fig. 4.4: $R a_{l}=10^{6}, A=8, h_{x} \times h_{y}=\frac{1}{30} \times \frac{8}{240} \quad$ y $\Delta t=10^{-2}$.

\section{2) Cavidad inclinada}

Con el objeto de observar el efecto que tiene el inclinar la cavidad rectangular un ángulo $\phi$ para un tiempo fijo, en la Figura 4.5 mostramos algunos resultados correspondientes a los ángulos $40^{\circ}, 130^{\circ}$ y $330^{\circ}$ para $R a_{l}=3.4 \times 10^{5}$ en $T=50$. Los resultados respectivos para el ángulo $0^{\circ}$ se pueden ver en la Figura 4.3. Hasta donde sabemos, estos resultados se están reportando por primera vez en este trabajo.

Para los ángulos $40^{\circ}$ y $130^{\circ}$, las isotermas parecen agudizar su estiramiento hacia algunas de las esquinas de la cavidad; en el caso de las líneas de corriente, se observa que si bien las celdas secundarias en el fondo y en la parte superior de la cavidad están siempre presentes, para cualquiera de los ángulos que se muestran, éstas crecen en algunos casos y en otros disminuyen su tamaño; además, cuando el ángulo es de $0^{\circ}$, Fig. 4.3, dos celdas secundarias adicionales se encuentran presentes cerca de las paredes laterales, las 

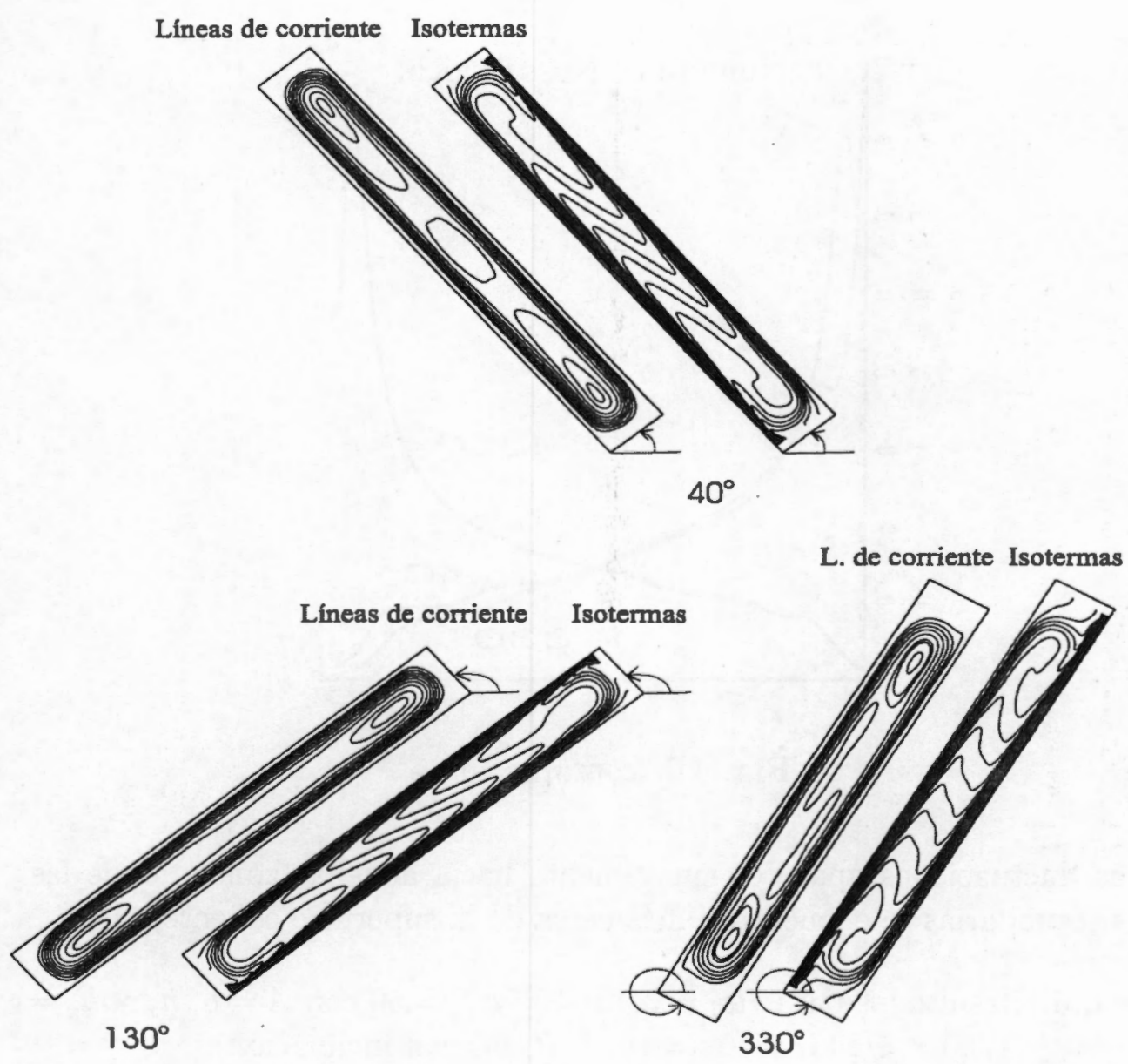

Fig. 4.5: $R a_{l}=3.4 \times 10^{5}, A=8, h_{x} \times h_{y}=\frac{1}{30} \times \frac{8}{240}, \Delta t=10^{-2}$ y $T=50$.

cuales desaparecen para $40^{\circ}$ y en cambio se forma una celda secundaria más en el centro de la cavidad; al aumentar el ángulo de inclinación hasta $130^{\circ}$, la celda secundaria del centro desaparece al fundirse con algunos contornos provenientes de las otras celdas secundarias localizadas en los extremos de la región; por último, para $330^{\circ}$ surgen dos nuevas celdas secundarias que se alargan hacia la zona central de la cavidad.

La respectiva gráfica del número de Nusselt local, Fig. 4.6, tomando en cuenta la de la Figura 4.3, correspondiente a $\phi=0^{\circ}$ en $T=50$, muestra nuevamente que la máxima transferencia de calor se alcanza en la parte inferior de la pared caliente y disminuye al ascender sobre la misma para los ángulos $\phi=0^{\circ}, \phi=40^{\circ}$ y $\phi=330^{\circ}$ y la situación se invierte para $\phi=130^{\circ}$. 


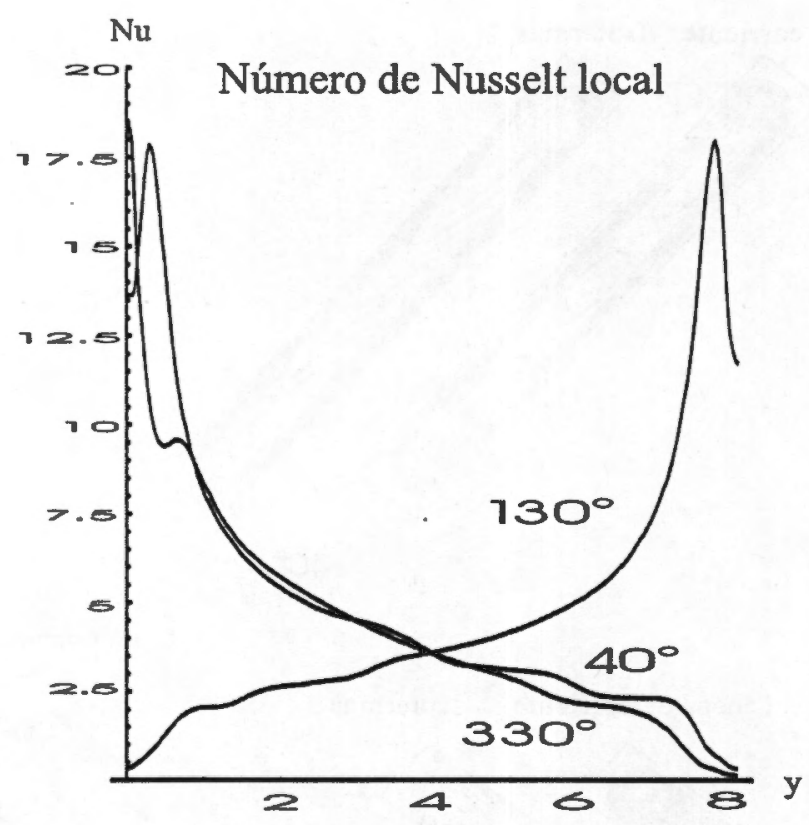

Fig. 4.6: continuación

Ligeras fluctuaciones aparecen nuevamente hacia aquellas zonas donde las celdas secundarias se encuentran más cerca de la superficie caliente,

Tabla L.5. Resultados para $R a_{l}=3.4 \times 10^{5}$ y $T=50$ con $A=8, h_{x} \times h_{y}=$ $1 / 30 \times 8 / 240$ y $\Delta t=10^{-2}$. (Caso con inclinación).

\begin{tabular}{|c|c|c|}
\hline$\phi$ & $\psi_{\min / \text { max }}$ & $\overline{N u}$ \\
\hline $0^{\circ}$ & -0.1498 & 4.8471 \\
$40^{\circ}$ & -0.1725 & 4.8046 \\
$130^{\circ}$ & 0.1745 & 4.6573 \\
$330^{\circ}$ & -0.1325 & 4.3867 \\
\hline
\end{tabular}

En la Tabla L.5 se resumen los valores extremos de la función de corriente así como el número de Nusselt global, en donde podemos observar que la inclinación de la cavidad provoca que el fluido tenga un movimiento más fuerte para ciertos ángulos, $40^{\circ}$ y $130^{\circ}$, girando en dirección negativa y positiva respectivamente, sin embargo la transferencia de calor sobre la pared caliente, medida por $\overline{N u}$, es mayor cuando no existe inclinación, $\phi=0^{\circ}$, aunque la diferencia con la que se obtiene para cualquiera de los otros ángulos es relativamente pequeña. 


\subsection{Esquema numérico en medios porosos}

El desarrollo del respectivo esquema numérico para medios porosos en términos de las variables secundarias, es muy similar al caso de medios libres con las diferencias naturales debido a la ecuación de momento correspondiente a cada medio, lo cual conducirá en el caso de medios porosos a un sistema de ecuaciones más sencillo que en el de medios libres, en el que no aparece la ecuación para la vorticidad y por lo tanto el número de ecuaciones a resolver es menor.

Una vez que se aplica la discretización en el tiempo, dada por la relación (3.1), se llega al siguiente sistema semidiscreto

$$
\begin{array}{rr}
\alpha \theta^{n+1}-\nabla^{2} \theta^{n+1}+\mathbf{u}^{n+1} \cdot \nabla \theta^{n+1}=f_{\theta} & \text { en } \Omega, \\
B \theta^{n+1}=0 & \text { sobre } \Gamma ; \\
\nabla^{2} \psi^{n+1}=-R a_{p}\left(\cos \phi{\left.\frac{\partial \theta}{\partial x+1}-\operatorname{sen} \phi \partial^{n y+1}\right)}^{n+1}=0\right. & \text { en } \Omega, \\
\psi^{n+1}=0 & \text { sobre } \Gamma
\end{array}
$$

donde nuevamente $n \geq 1, \alpha=\frac{3}{2 \Delta t}, f_{\theta}=\frac{4 \theta^{n}-\theta^{n-1}}{2 \Delta t}, \mathbf{u}=\left(u_{1}, u_{2}\right)$ está dada por (4.1) en términos de $\psi$, y B es el operador de frontera mixto para la temperatura.

Al renombrar $\left(\psi^{n+1}, \theta^{n+1}\right)$ por $(\psi, \theta)$ el sistema estacionario de ecuaciones que debemos resolver en cada nivel de tiempo se simplifica en la forma

$$
\begin{array}{rlrl}
\nabla^{2} \psi=-R a_{p}\left(\cos \phi \frac{\partial \theta}{\partial x}-\operatorname{sen} \phi \frac{\partial \theta}{\partial y}\right) & & \text { en } \Omega, \\
\psi & =0 & & \text { sobre } \Gamma ; \\
\alpha \theta-\nabla^{2} \theta+\mathbf{u} \cdot \nabla \theta & =f_{\theta} & & \text { en } \Omega, \\
B \theta & =0 & & \text { sobre } \Gamma
\end{array}
$$

Usando la relación (4.13) se tiene que el sistema (4.22) es equivalente a

$$
\begin{array}{llrl}
\nabla^{2} \psi=-R a_{p}\left(\cos \phi \frac{\partial \theta}{\partial x}-\operatorname{sen} \phi \frac{\partial \theta}{\partial y}\right) & \text { en } \Omega, & \psi=0 & \text { sobre } \Gamma, \\
\Theta(\theta, \psi)=0 & \text { en } \Omega, & B \theta=0 & \text { sobre } \Gamma
\end{array}
$$

A partir de este sistema y conocidos $\left(\psi^{\circ}, \theta^{\circ}\right)$, aplicamos el siguiente método iterativo

$$
\begin{aligned}
& \nabla^{2} \psi^{m+1}=-R a_{p}\left(\cos \phi{\frac{\partial \theta^{m}}{\partial x}}^{m}-\operatorname{sen} \phi_{\frac{\theta}{\partial y}^{m}}\right) \text { en } \Omega, \quad B \theta=0 \text { sobre } \Gamma, \\
& \theta^{m+1}=\theta^{m}-\lambda\left(\alpha-\nabla^{2}\right)^{-1} \Theta\left(\theta^{m}, \psi^{m+1}\right) \text { en } \Omega, \quad B \theta=0 \text { sobre } \Gamma \text {. }
\end{aligned}
$$


Resolvemos hasta alcanzar la convergencia y, cuando esto ocurre, tomamos $\left(\psi^{n+1}, \theta^{n+1}\right)=\left(\psi^{m+1}, \theta^{m+1}\right)$. Los valores de $\left(\psi^{1}, \theta^{1}\right)$ que se requieren en el proceso, se obtienen de manera similar a como se indicó para medios libres.

Finalmente señalamos que la segunda ecuación del sistema (4.24) puede sustituirse por (4.17), con $\gamma=1$, y los dos problemas elípticos que resultan del proceso los resolvemos nuevamente usando Fishpack, con la opción de segundo orden.

\subsubsection{Resultados numéricos}

Presentamos experimentos numéricos para flujos de estado estacionario que se desarrollan en cavidades rectangulares, donde nuevamente la condición de frontera para la función de corriente es $\psi=0$, y para la temperatura está dada por

$$
\left.\frac{\partial \theta}{\partial n}\right|_{y=0, b}=\left.0 \quad \theta\right|_{x=0}=\left.0.5 \quad \theta\right|_{x=a}=-0.5
$$

en las primeras dos subsecciones, donde se consideran cavidades cuadradas y rectangulares verticales; mientras que en la tercera subsección, cavidades rectangulares horizontales, se considera la siguiente condición de frontera

$$
\left.\frac{\partial \theta}{\partial n}\right|_{x=0, a}=\left.0 \quad \cdot \theta\right|_{y=0}=\left.1 \quad \theta\right|_{y=b}=0 .
$$

Además, en cualesquiera de los casos considerados, las condiciones iniciales respectivas son $\psi(\mathbf{x}, 0)=0$ y $\theta(\mathbf{x}, 0)=0$. Nuevamente, el parámetro del proceso iterativo es $\lambda=0.7$ y la tolerancia para determinar $T_{\text {est }}$, a partir del criterio dado por (3.9), es de $10^{-5}$. Los experimentos numéricos involucran una variedad amplia de: razones geométricas, ángulos de inclinación y de valores del número de Rayleigh. Algunos de los resultados que se presentan se comparan con los obtenidos por otros autores con el objeto de validar el esquema, mientras que otros son resultados nuevos que se obtuvieron realizando los correspondientes estudios de independencia de malla y de paso de tiempo, considerando para ello el error relativo discreto, descrito por las relaciones (4.19) y (4.20).

En algunos resultados se especifican los valores de los contornos mientras que en otros se obtienen por defecto. Nuevamente, los resultados se complementan con los respectivos números de Nusselt, local y global, sobre la frontera caliente y con los valores extremos de la función de corriente $\psi$. Los 
respectivos parámetros de discretización, el paso de tiempo $\Delta t$ y el tamaño de la malla $h_{x} \times h_{y}$, se especifican en cada caso.

1) Cavidad cuadrada $(a=1=b)$

Los resultados corresponden a diversos valores del número de Rayleigh entre $10^{2} \leq R a_{p} \leq 10^{4}$ y una variedad de ángulos entre $0^{\circ} \leq \phi \leq 360^{\circ}$. Muchos de estos resultados son obtenidos por Baytas [29], resolviendo el problema no estacionario con otro esquema numérico.

La Figura 4.7 muestra algunos resultados para $R a_{p}=10^{2}$ y $0^{\circ} \leq \phi \leq 90^{\circ}$. $\mathrm{Al}$ considerar este rango de ángulos reparamos desde el calentamiento lateral hasta el calentamiento en la frontera inferior, de la cavidad. Podemos observar que, para todos los ángulos, las líneas de corriente forman una única celda principal girando en dirección negativa, los valores que muestran los contornos indican que el movimiento del fluido es un poco más lento cuando el calentamiento es lateral $\left(\phi=0^{\circ}\right)$. Por otro lado, las isotermas se concentran, en mayor o menor medida, cerca de la parte inferior de la pared, indicando mayor transferencia de calor en esta zona. La gráfica del número de Nusselt local manifiesta que en la parte inferior de la superficie caliente, la transferencia de calor es mayor cuando $\phi=40^{\circ}$ y mucho menor cuando la cavidad se calienta por debajo, $\phi=90^{\circ}$, sin embargo, ésta última desciende lentamente en comparación a lo que sucede para los otros valores de $\phi$ considerados, e incluso en la mitad superior de la pared es casi igual o mayor que la de los otros ángulos. Estos resultados concuerdan perfectamente con los obtenidos por Baytas [29], con excepción del correspondiente para $\phi=60^{\circ}$ ya que este ángulo no lo considera.

Tabla P.1. Resultados para $R a_{p}=10^{2}, A=1$,

$$
h=\frac{1}{30} \text { y } \Delta t=10^{-2} \text {. }
$$

\begin{tabular}{|c|c|c|c|}
\hline$\phi$ & $\psi_{\min }$ & $\overline{N u}$ & $T_{\text {est }}$ \\
\hline $0^{\circ}$ & -4.6639 & 3.3865 & 0.16 \\
$40^{\circ}$ & -6.4561 & 4.1775 & 0.25 \\
$60^{\circ}$ & -6.5817 & 3.8961 & 0.31 \\
$90^{\circ}$ & -5.3464 & 2.6805 & 0.73 \\
\hline
\end{tabular}

A partir de los valores de $\psi_{\min }$ y $\overline{N u}$, Tabla P.1, puede verse que aunque el movimiento del fluido es más fuerte para $\phi=60^{\circ}$ y menor en $\phi=0^{\circ}$, la transferencia de calor global es mayor cuando $\phi=40^{\circ}$ mientras que la 

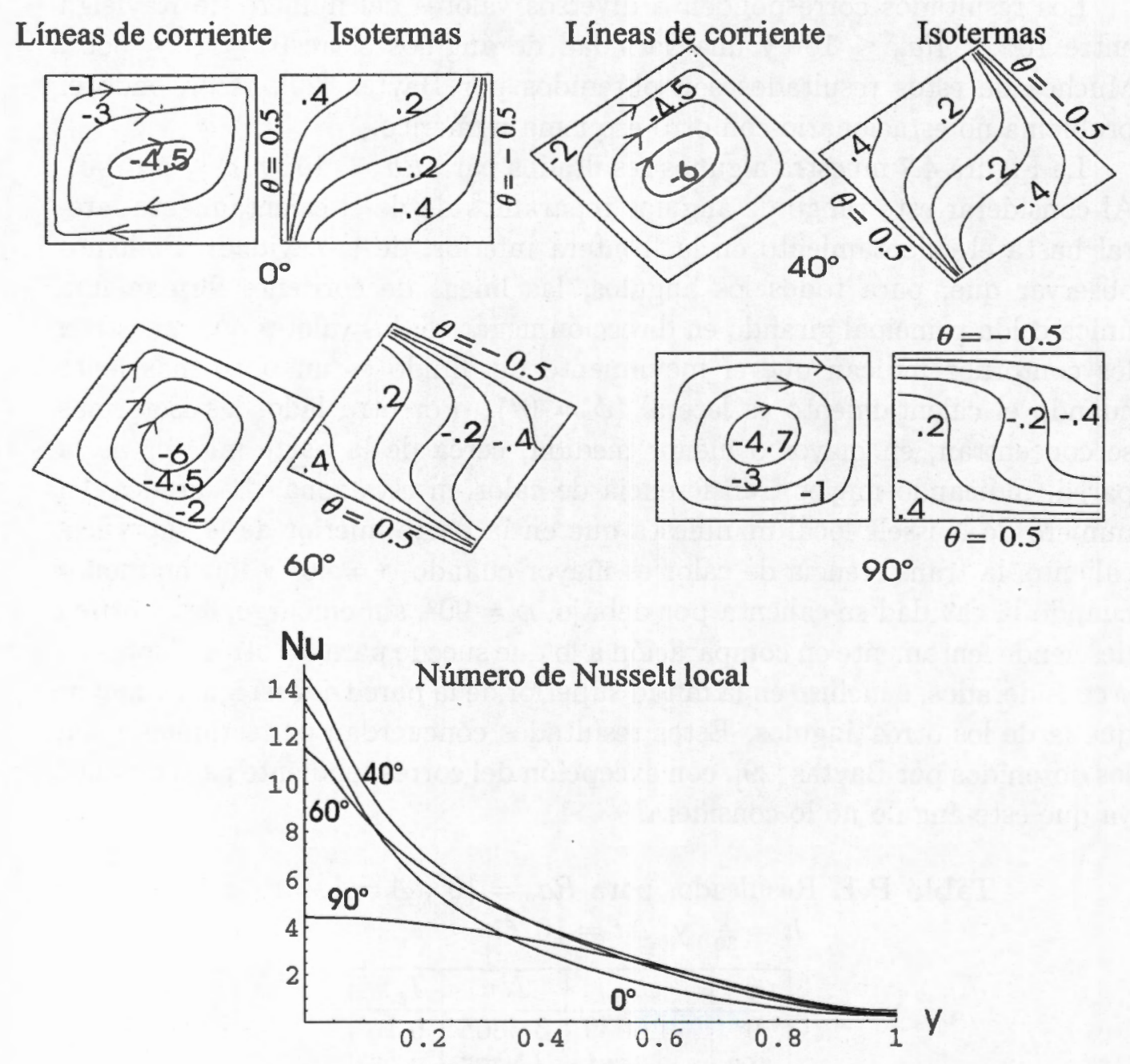

Fig. 4.7: $R a_{p}=10^{2}, A=1, h=\frac{1}{30}$ y $\Delta t=10^{-2}$. 
menor se tiene cuando $\phi=90^{\circ}$. Por lo que respecta a los valores de $t_{\text {est }}$, se observa que el tiempo que se requiere para que el fluido se estacione es menor cuando la cavidad se calienta lateralmente y aumenta con el ángulo, de esta manera, cuando el calentamiento se da por debajo el flujo tarda más tiempo en estacionarse. Saeid y Pop [30], indican que $\overline{N u}=3.002$ para el caso de $\phi=0^{\circ}$, que es un poco menor que el que se obtiene aquí.

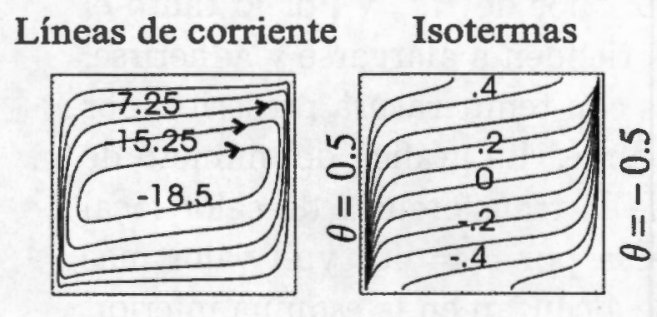

$0^{\circ}$
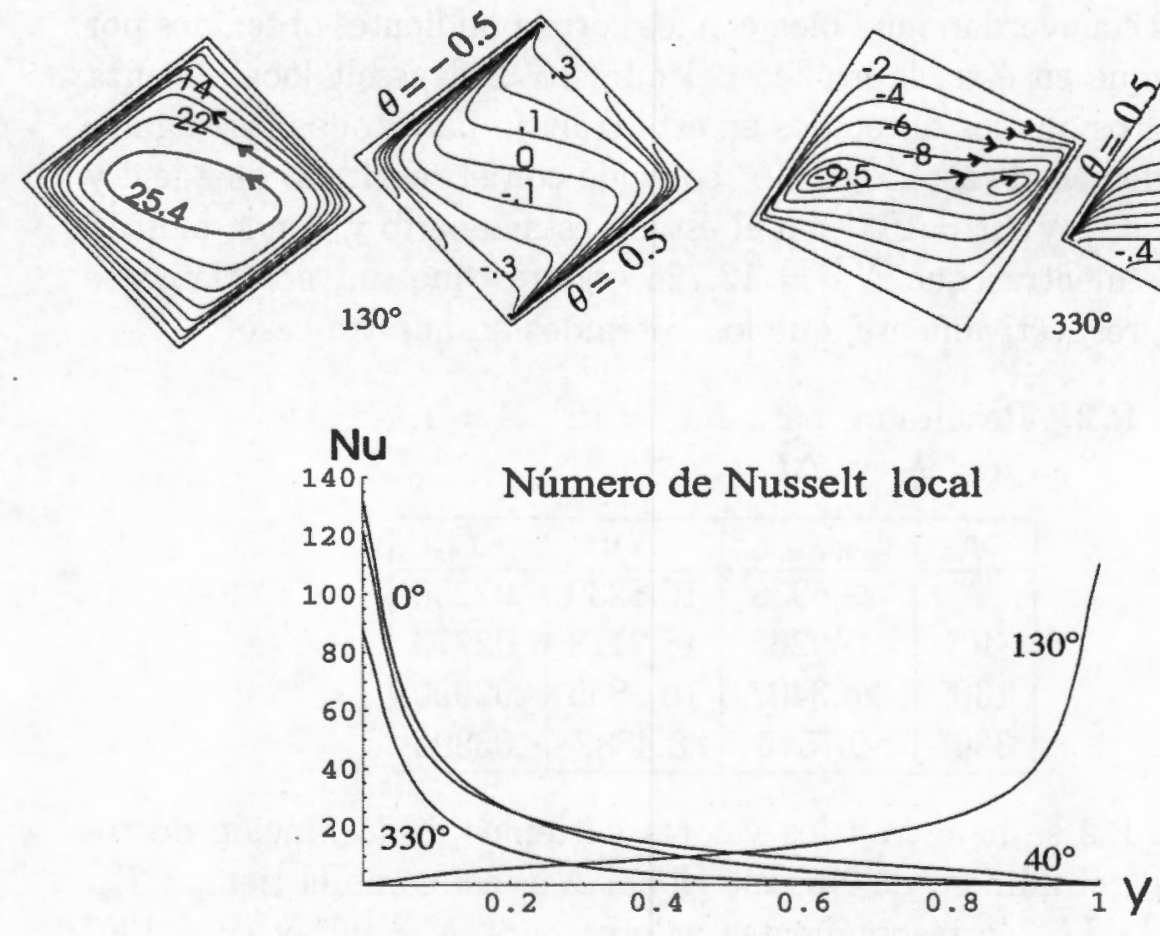

Fig. 4.8: $R a_{p}=10^{3}, A=1, h=\frac{1}{70}$ y $\Delta t=10^{-5}$.

Cuando $R a_{p}=10^{3}$, fue necesario reducir el tamaño de malla, $h=\frac{1}{70}, y$ el paso de tiempo, $\Delta t=10^{-5}$. En la Figura 4.8 se muestran los resultados obtenidos para $0^{\circ} \leq \phi \leq 360^{\circ}$. Cuando $\phi=0^{\circ}$, el fluido caliente proveniente

\section{Líneas de corriente Isotermas}
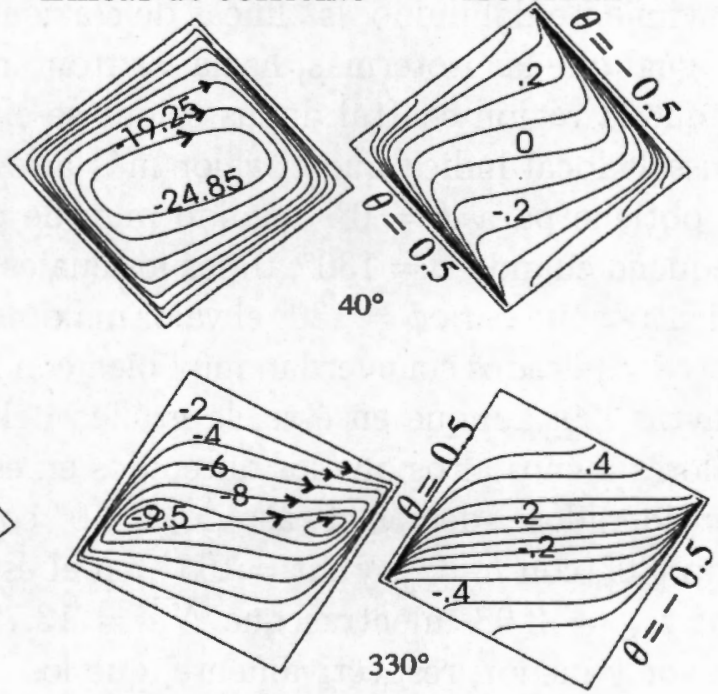
de la frontera izquierda que asciende y el frío de la frontera derecha que cae genera una celda que rota en dirección negativa. Una situación similar se presenta cuando $\phi=40^{\circ}$ y $\phi=330^{\circ}$, y contraria cuando $\phi=130^{\circ}$; además en algunos ángulos aparecen celdas secundarias, como se observa para $\phi=330^{\circ}$. De los valores que alcanzan las líneas de corriente, se tiene que el movimiento del fluido adquiere mayor fuerza cuando $\phi=130^{\circ}$ y $\phi=40^{\circ}$, y es menor cuando $\phi=330^{\circ}$; al aumentar el valor de $R a_{p}$ y por lo tanto el movimiento del fluido, las líneas de corriente tienden a alargarse y adherirse, al igual que las isotermas, hacia las fronteras con temperatura prescrita, por lo que la región central de las celdas se expande. La gráfica del número de Nusselt local indica que el valor más alto de la transferencia de calor local se obtiene para $\phi=0^{\circ}$, seguido muy de cerca por $\phi=40^{\circ}$ y el valor más pequeño cuando $\phi=130^{\circ}$, todos los cuales se alcanzan en la esquina inferior, mientras que para $\phi=130^{\circ}$ el valor máximo se alcanza en la esquina superior. Estos resultados concuerdan muy bien con los correspondientes obtenidos por Baytas [29] aunque en éste, la gráfica del número de Nusselt local alcanza valores menos altos que los obtenidos en este trabajo para todos los ángulos considerados; además el caso de $\phi=0^{\circ}$ coincide con el resultado de Saeid y Pop [30], con $h=\frac{1}{40}$ y $\Delta t=10^{-4}$, en el estado estacionario y donde indican que $t_{\text {est }}=0.08$ mientras que $\overline{N u}=13.726$, valores que son notoriamente mayor y menor, respectivamente, que los obtenidos en nuestro caso.

Tabla P.2. Resultados para $R a_{p}=10^{3}, A=1$,

$$
h=\frac{1}{70} \text { y } \Delta t=10^{-5} \text {. }
$$

\begin{tabular}{|c|c|c|c|}
\hline$\phi$ & $\psi_{\min / \operatorname{máx}}$ & $\overline{N u}$ & $T_{\text {est }}$ \\
\hline $0^{\circ}$ & -19.5938 & 16.8230 & .02250 \\
$40^{\circ}$ & -25.9263 & 18.2113 & .02779 \\
$130^{\circ}$ & 26.3407 & 16.9855 & .02920 \\
$330^{\circ}$ & -9.7545 & 8.4882 & .03396 \\
\hline
\end{tabular}

En la Tabla P.2 se muestran los valores extremos de la función de corriente, $\psi_{m i n / m a x}$, el número de Nusselt global $\overline{N u}$, así como el tiempo $T_{e s t}$ para $R a_{l}=10^{3}$. Los correspondientes valores para $\phi=40^{\circ}$ y $\phi=130^{\circ}$ muestran que tanto el movimiento del fluido como la transferencia de calor global son mayores a los de $\phi=0^{\circ}$ y $\phi=330^{\circ}$; en particular, el movimiento más fuerte del fluido se tiene cuando $\phi=130^{\circ}$, y la mayor transferencia de calor cuando $\phi=40^{\circ}$, mientras que el tiempo $t_{\text {est }}$ es menor para $\phi=0^{\circ} \mathrm{y}$ aumenta conforme el ángulo se incrementa. 

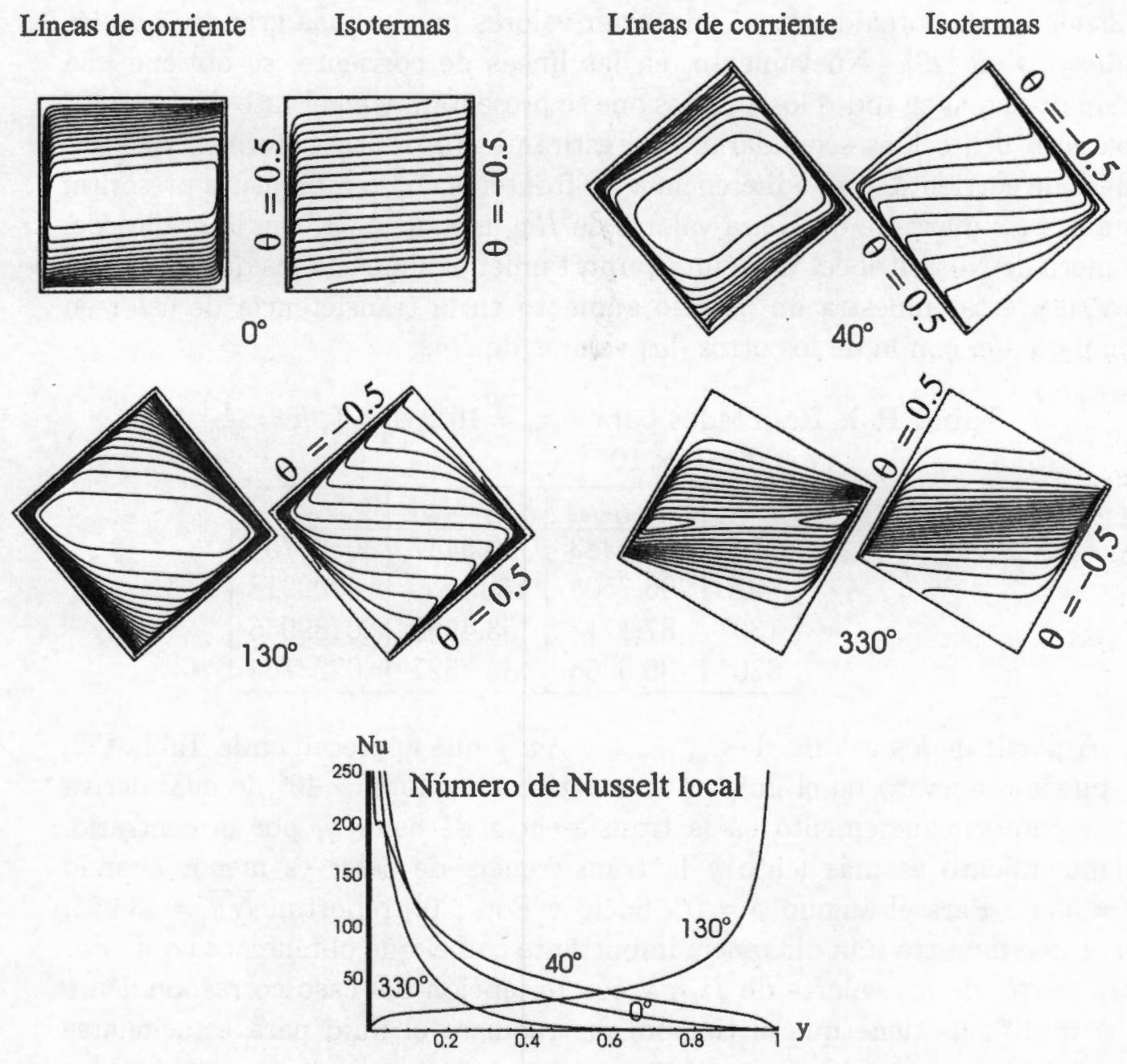

Fig. 4.9: $R a_{p}=10^{4}, A=1, h=1 / 300$ y $\Delta t=2 \times 10^{-7}$. 
Cuando $R a_{p}=10^{4}$, Fig. 4.9, fue necesario disminuir todavía más el tamaño de la malla hasta $h=\frac{1}{300}$ y el paso de tiempo a $\Delta t=2 \times 10^{-7}$. Los resultados obtenidos nuevamente concuerdan con los correspondientes mostrados por Baytas [29], con la particularidad de que, de manera similar a lo ocurrido para $R a_{p}=10^{3}$, las correspondientes curvas del número de Nusselt local, obtenidas aquí, alcanzan valores mucho más grandes que los indicados en [29]. Nuevamente, en las líneas de corriente, se obtiene una celda principal en todos los ángulos que se presentan, y en el caso de $\phi=330^{\circ}$ aparecen dos celdas secundarias. El estiramiento de las isotermas y de las líneas de corriente, y su adherencia a las fronteras con temperatura prescrita, es aún más marcada que para valores de $R a_{p}$ menores. Si bien la gráfica del número de Nusselt local tiene un comportamiento similar a las que ya se han descrito, éstas muestra un notorio aumento en la transferencia de calor en comparación con la de los otros dos valores de $R a_{p}$.

Tabla P.3. Resultados para $R a_{p}=10^{4}, A=1, h=\frac{1}{300}$ y $\Delta t=2 \times 10^{-7}$.

\begin{tabular}{|c|c|c|c|}
\hline$\phi$ & $\psi_{\min / \max x}$ & $\overline{N u}$ & $T_{\text {est }}$ \\
\hline $0^{\circ}$ & -69.2383 & 59.5447 & .015575 \\
$40^{\circ}$ & -96.7505 & 150.3877 & .0009918 \\
$130^{\circ}$ & 87.1717 & 53.4990 & .0189956 \\
$330^{\circ}$ & -36.6366 & 33.7527 & .0283764 \\
\hline
\end{tabular}

A partir de los valores de $\psi_{\min / \max x}$ y $\overline{N u}$ y que aparecen en la Tabla P.3, se puede observar que el flujo es más activo cuando $\phi=40^{\circ}$, lo cual deriva en un notorio incremento en la transferencia de calor y, por el contrario, el movimiento es más lento y la transferencia de calor es menor cuando $\phi=330^{\circ}$. Para el ángulo $\phi=0^{\circ}$, Saeid y Pop [30], reportan $\overline{N u}=43.953$, valor que muestra una diferencia importante con el que obtenemos aquí. Por otra parte, de los valores de $T_{e s t}$, y con excepción del caso correspondiente a $\phi=40^{\circ}$, se tiene que el tiempo que requiere el flujo para estacionarse aumenta con el ángulo; sin embargo, comparando con los tiempos obtenidos para los otros valores de $R a_{p}$ podemos concluir que cuando éste aumenta, el tiempo para que el flujo llegue al estado estacionario disminuye.

2) Cavidad rectangular vertical

En esta subsección presentamos resultados para $R a_{p}=10^{2}$ y $10^{3}$, con $A \geq$ 1 y $0^{\circ} \leq \phi \leq 360^{\circ}$. Cuando $R a_{p}=10^{2}$ y $A=4(a=1$ y $b=4)$, Fig. 4.10, 

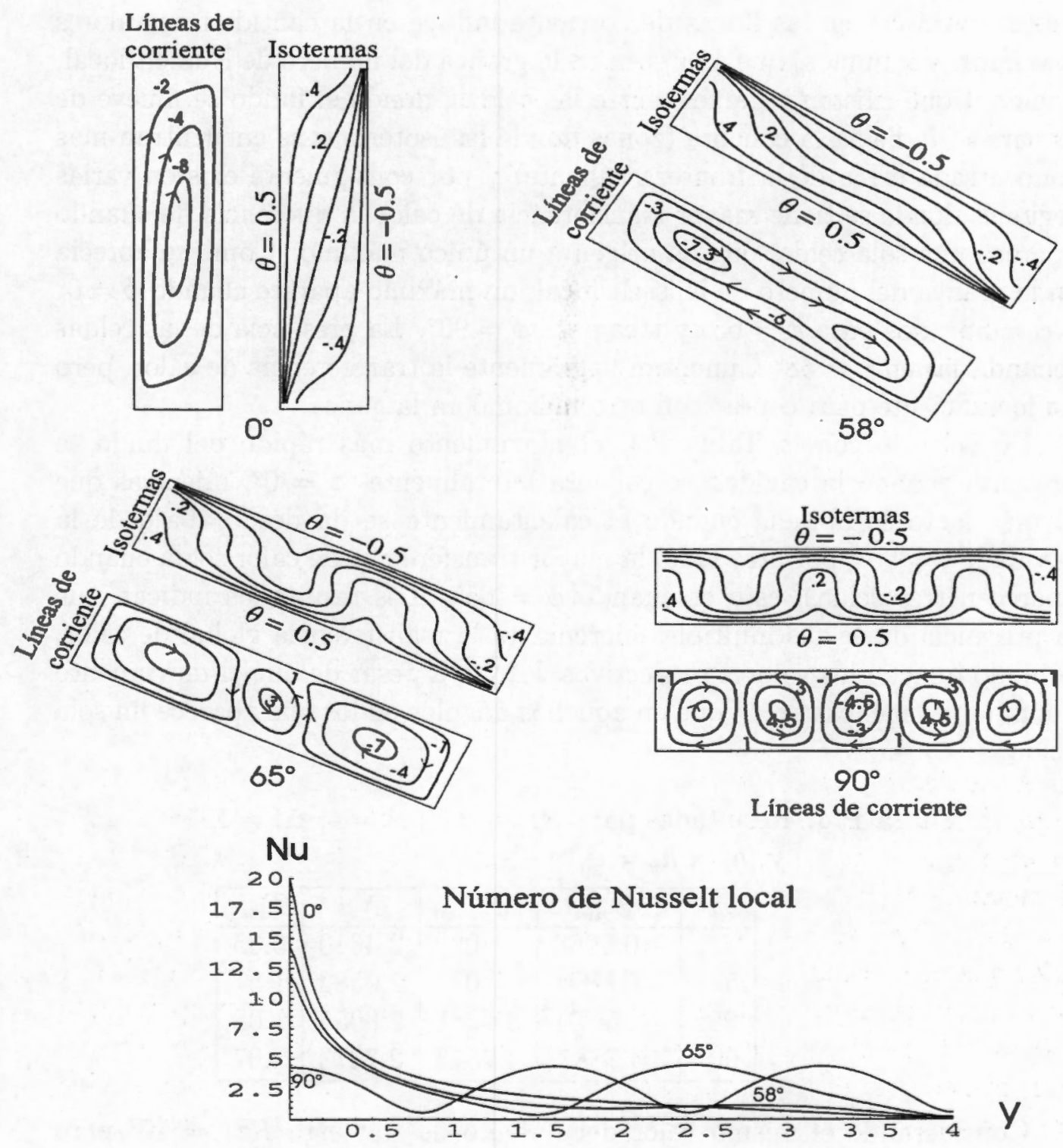

Fig. 4.10: $R a_{p}=10^{2}, A=4, h_{x} \times h_{y}=\frac{1}{30} \times \frac{4}{120}$ y $\Delta t=10^{-2}$. 
aparecen desde una celda para $\phi=0^{\circ}$ hasta cinco celdas primarias, rotando en direcciones opuestas una con respecto a las vecinas adyacentes, cuando la cavidad se calienta desde abajo, esto es cuando $\phi=90^{\circ}$. La presencia de celdas múltiples en las líneas de corriente influye en la cantidad de valores máximos (y mínimos) que aparecen en la gráfica del número de Nusselt local, debido a que existen regiones, entre las celdas, donde el fluido se mueve de la pared fría hacia la caliente (zonas donde las isotermas se encuentran más concentrada cerca de la frontera caliente) y por consiguiente existen varias regiones donde se tiene mayor transferencia de calor, a diferencia de cuando aparece una sola celda, que da origen a un único máximo. Como se aprecia en la gráfica del número de Nusselt local, un máximo aparece cuando $\phi=0^{\circ}$ у $\phi=58^{\circ}$, dos para $\phi=65^{\circ}$ y tres para $\phi=90^{\circ}$. La presencia de las celdas secundarias en $\phi=58^{\circ}$ aumentan ligeramente la transferencia de calor, pero no lo suficiente para contar con otro máximo en la gáfica.

De acuerdo con la Tabla P.4, el movimiento más rápido del fluido se presenta cuando la cavidad se calienta lateralmente, $\phi=0^{\circ}$, mientras que el más lento se obtiene cuando el calentamiento se da desde abajo de la cavidad, $\phi=90^{\circ}$; por otro lado, la mayor transferencia de calor se da cuando aparecen tres celdas, esto es cuando $\phi=65^{\circ}$. Los resultados indican que la presencia de celdas múltiples incrementa la transferencia global de calor, como lo indican los valores respectivos de $\overline{\mathrm{Nu}}$, a pesar de que el movimiento del fluido es menos rápido que en aquellos ángulos en los que aparece un sola celda.

Tabla P.4. Resultados para $R a_{p}=10^{2}, A=4, \Delta t=10^{-2}$ y $h_{x} \times h_{y}=\frac{1}{30} \times \frac{4}{120}$.

\begin{tabular}{|c|c|c|c|c|}
\hline$\phi$ & $\psi_{\min }$ & $\psi_{\operatorname{máx}}$ & $\overline{N u}$ & $T_{\text {est }}$ \\
\hline $0^{\circ}$ & -10.5968 & 0 & 2.4610 & 0.35 \\
$58^{\circ}$ & -7.7180 & 0 & 2.0582 & 1.57 \\
$65^{\circ}$ & -7.5070 & 3.4281 & 2.8055 & 1.08 \\
$90^{\circ}$ & -4.7887 & 4.7843 & 2.7273 & 2.07 \\
\hline
\end{tabular}

Considerando el mismo valor del número de Rayleigh $R a_{p}=10^{2}$ pero aumentando la razón geométrica hasta $A=8(a=1$ y $b=8)$, se observa de las líneas de corriente en la Figura 4.11 que ahora se obtienen desde una celda principal, nuevamente para el calentamiento lateral, $\phi=0^{\circ}$, hasta once para $\phi=90^{\circ}$, calentamiento desde el fondo. Por esta razón, la gráfica del número de Nusselt local muestra un solo máximo para $\phi=0^{\circ}$ y $50^{\circ}$, cinco cuando 


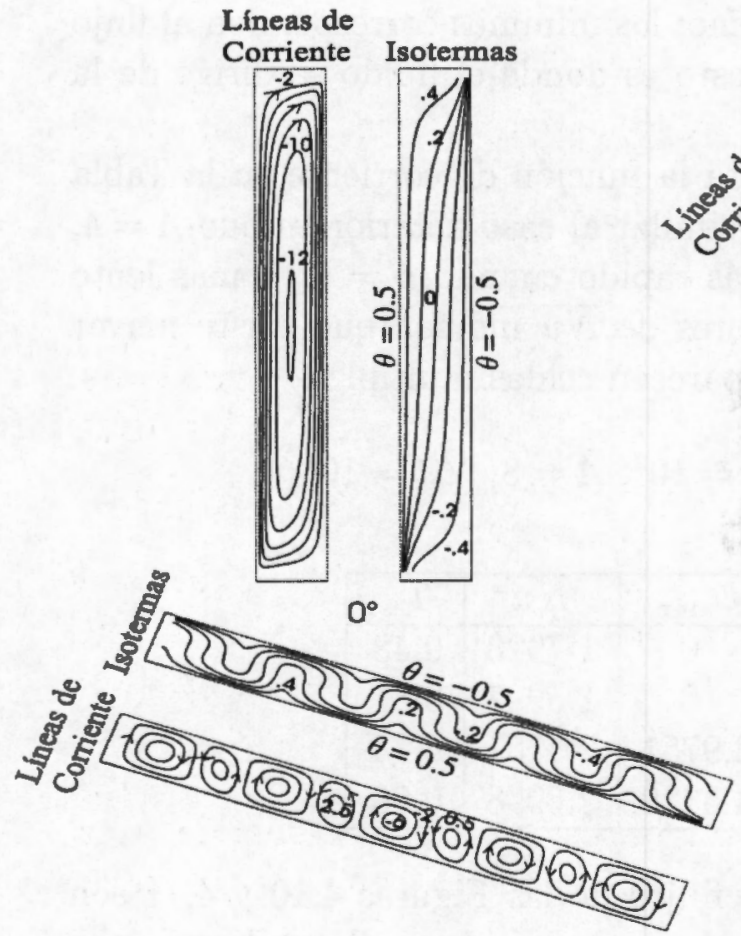

$70^{\circ}$
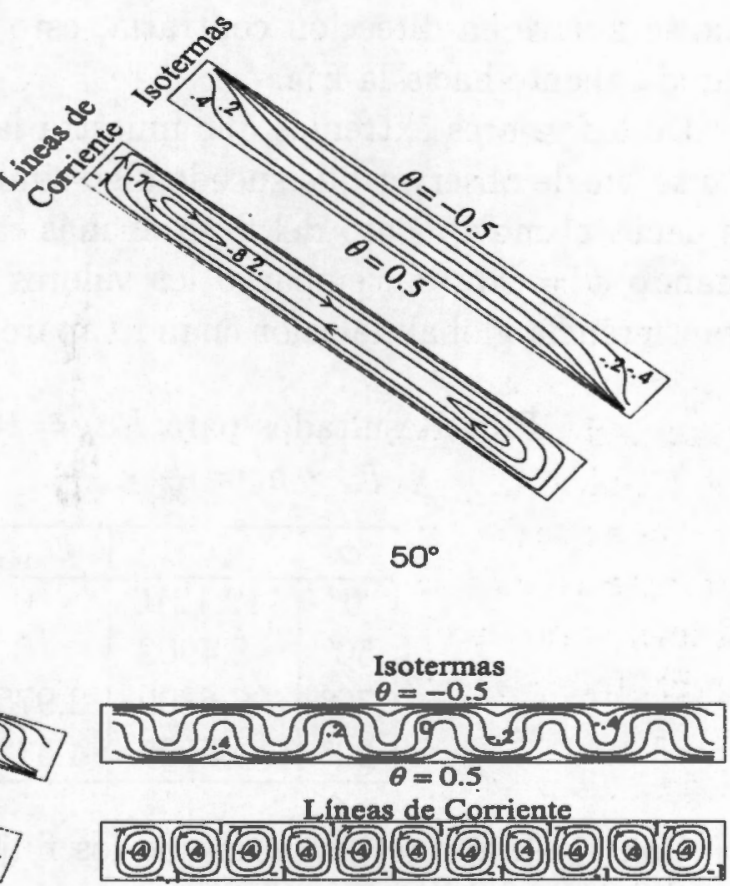

$90^{\circ}$

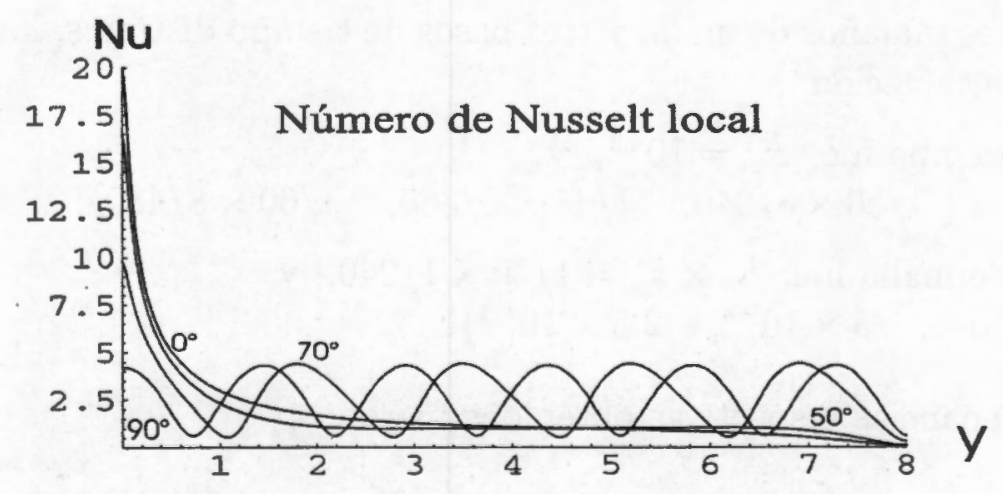

Fig. 4.11: $R a_{p}=10^{2}, A=8, h_{x} \times h_{y}=\frac{1}{30} \times \frac{8}{240}$ y $\Delta t=10^{-2}$. 
$\phi=70^{\circ}$ y seis para $\phi=90^{\circ}$, esto es, al incrementar la razón geométrica, la cantidad de celdas múltiples aumenta, dependiendo también del ángulo, con lo cual existirán entonces más regiones sobre la frontera caliente donde la transferencia de calor alcanza un máximo; los mínimos corresponden al flujo que se mueve en dirección contraria, esto es donde el fluido se dirige de la pared caliente hacia la fría.

De los valores extremos que muestra la función de corriente en la Tabla P.5 se puede observar que sucede algo similar al caso anterior en que $A=4$, es decir, el movimiento del flujo es más rápido cuando $\phi=0^{\circ}$ y más lento cuando $\phi=90^{\circ}$, sin embargo los valores de $\overline{N u}$ indican que existe mayor transferencia global de calor cuando aparecen celdas múltíples.

P.5. Resultados para $R a_{p}=10^{2}, A=8, \Delta t=10^{-2}$ y $h_{x} \times h_{y}=\frac{1}{30} \times \frac{8}{240}$.

\begin{tabular}{|c|c|c|c|c|}
\hline$\phi$ & $\psi_{\min }$ & $\psi_{\text {máx }}$ & $\overline{N u}$ & $T_{\text {est }}$ \\
\hline $0^{\circ}$ & -12.1216 & 0 & 1.7923 & 0.48 \\
$50^{\circ}$ & -8.4909 & 0 & 1.5997 & 0.81 \\
$70^{\circ}$ & -6.6809 & 2.9751 & 2.7701 & 6.74 \\
$90^{\circ}$ & -4.5097 & 4.5329 & 2.6998 & 0.56 \\
\hline
\end{tabular}

Con el objeto de justificar que los flujos de las Figuras 4.10 y 4.11 son correctos, se realizaron estudios de independencia de malla y de paso de tiempo para la cavidad vertical, esto es cuando $\phi=0^{\circ}$; cuando $A=8 \mathrm{se}$ emplearon tres tamaños de malla y tres pasos de tiempo distintos, los que se detallan a continuación

(1) paso de tiempo fijo: $\Delta t=10^{-2}, \mathrm{y}$

$$
h_{x} \times h_{y}=\{1 / 30 \times 8 / 240,1 / 45 \times 8 / 360,1 / 60 \times 8 / 480\} ;
$$

(2) tamaño de malla fija: $h_{x} \times h_{y}=1 / 30 \times 1 / 240, y$

$$
\Delta t=\left\{10^{-2}, \quad 5 \times 10^{-3}, \quad 2.5 \times 10^{-3}\right\} .
$$

Las discrepancias respectivas obtenidas fueron:

(1) menos del $6 \times 10^{-2} \%$ (a lo más $3.8 \times 10^{-1} \%$ para la función de corriente y $5.9 \times 10^{-1} \%$ para la temperatura);

(2) menos del $5 \times 10^{-2} \%\left(2.44 \times 10^{-2} \%\right.$ para la función de corriente y $4.96 \times$ $10^{-2} \%$ para la temperatura). 
El valor mínimo alcanzado por la función de corriente $\psi$ en cada caso (el máximo siempre es cero) es:

(1) $\min =-12.1216,-12.1206,-12.1280$;

(2) $\min =-12.1216,-12.1223,-12.1238$.

De esta manera, los resultados mostrados en la Figura 4.11 se consideran como correctos.

Los estudios correspondientes para el caso donde $A=4$, muestran un comportamiento muy similar.

Por otra parte, los resultados obtenidos para $R a=10^{3}, A=4$ y ángulos entre $0^{\circ} \leq \phi \leq 360^{\circ}$ se muestran en la Figura 4.12; puede observarse que cuando $\phi=0^{\circ}, \phi=65^{\circ}$ y $\phi=300^{\circ}$ aparece una única celda, alargada y que ocupa toda la cavidad, rotando en dirección negativa en los tres casos. Algunos experimentos adicionales permitieron determinar que la existencia de celdas múltiples sucede cuando $66^{\circ} \leq \phi \leq 114^{\circ}$, un ejemplo de ello se da cuando $\phi=110^{\circ}$ y en donde aparecen siete celdas primarias, dos de las cuales se localizan en los extremos de la cavidad y son de mayor tamaño y las otras cinco, claramente más pequeñas, ocupando la región central limitada por las grandes; la gráfica del número de Nusselt local para este ángulo nuevamente indica que la existencia de otros máximos es determinada por la presencia de celdas múltiples.

Tabla P.6. Resultados para $R a_{p}=10^{3}, A=4, \Delta t=10^{-5}$

$\mathrm{y} h_{x} \times h_{y}=\frac{1}{70} \times \frac{4}{280}$.
\begin{tabular}{|c|c|c|c|c|}
\hline$\phi$ & $\psi_{\min }$ & $\psi_{\max }$ & $\overline{N u}$ & $t_{\text {est }}$ \\
\hline $0^{\circ}$ & -44.6267 & 0 & 9.1802 & .02381 \\
$65^{\circ}$ & -37.2395 & 0 & 7.0151 & .09325 \\
$110^{\circ}$ & -16.2286 & 29.8881 & 10.2587 & .10000 \\
$300^{\circ}$ & -20.1421 & 0 & 4.0483 & .05061 \\
\hline
\end{tabular}

En la Tabla P.6 se puede advertir que, al igual de lo que sucedió en el caso anterior con $R a_{p}=10^{2}$, el movimiento mayor del fluido ocurre cuando $\phi=0^{\circ}$, en donde se obtiene una única celda, sin embargo, existe mayor transferencia de calor cuando aparecen celdas múltiples.

En la Figura 4.13 se muestran los resultados obtenidos para este mismo número de Rayleigh cuando aumentamos la razón geométrica a $A=8$ y para ángulos entre $0^{\circ} \leq \phi \leq 360^{\circ}$. Como se puede notar, para $\phi=117^{\circ}$, cuando aparecen celdas múltiples, el movimiento del fluido adquiere una forma más 

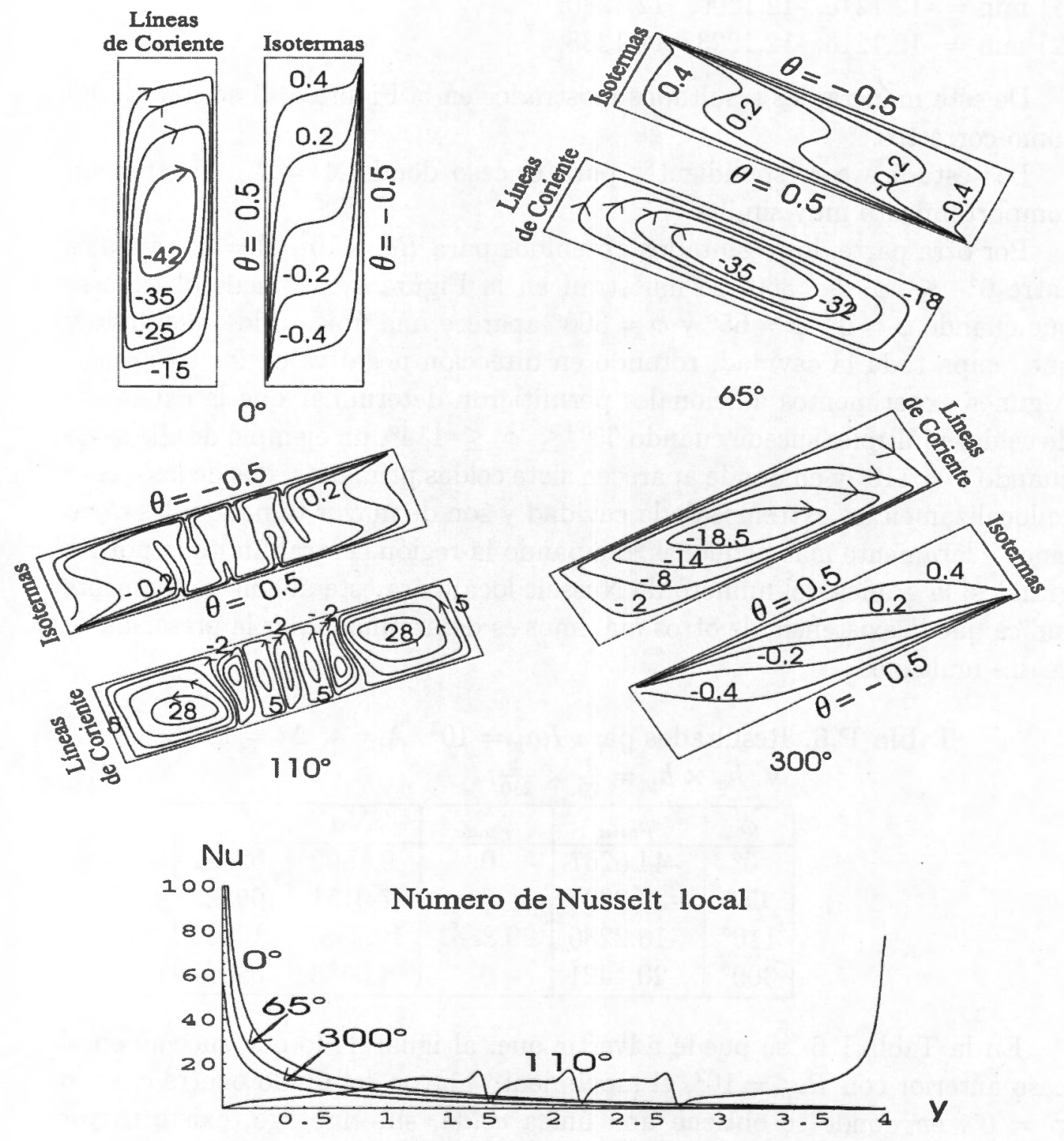

Fig. 4.12: $R a_{p}=10^{3}, A=4, h_{x} \times h_{y}=\frac{1}{70} \times \frac{4}{280}$ y $\Delta t=10^{-5}$. 

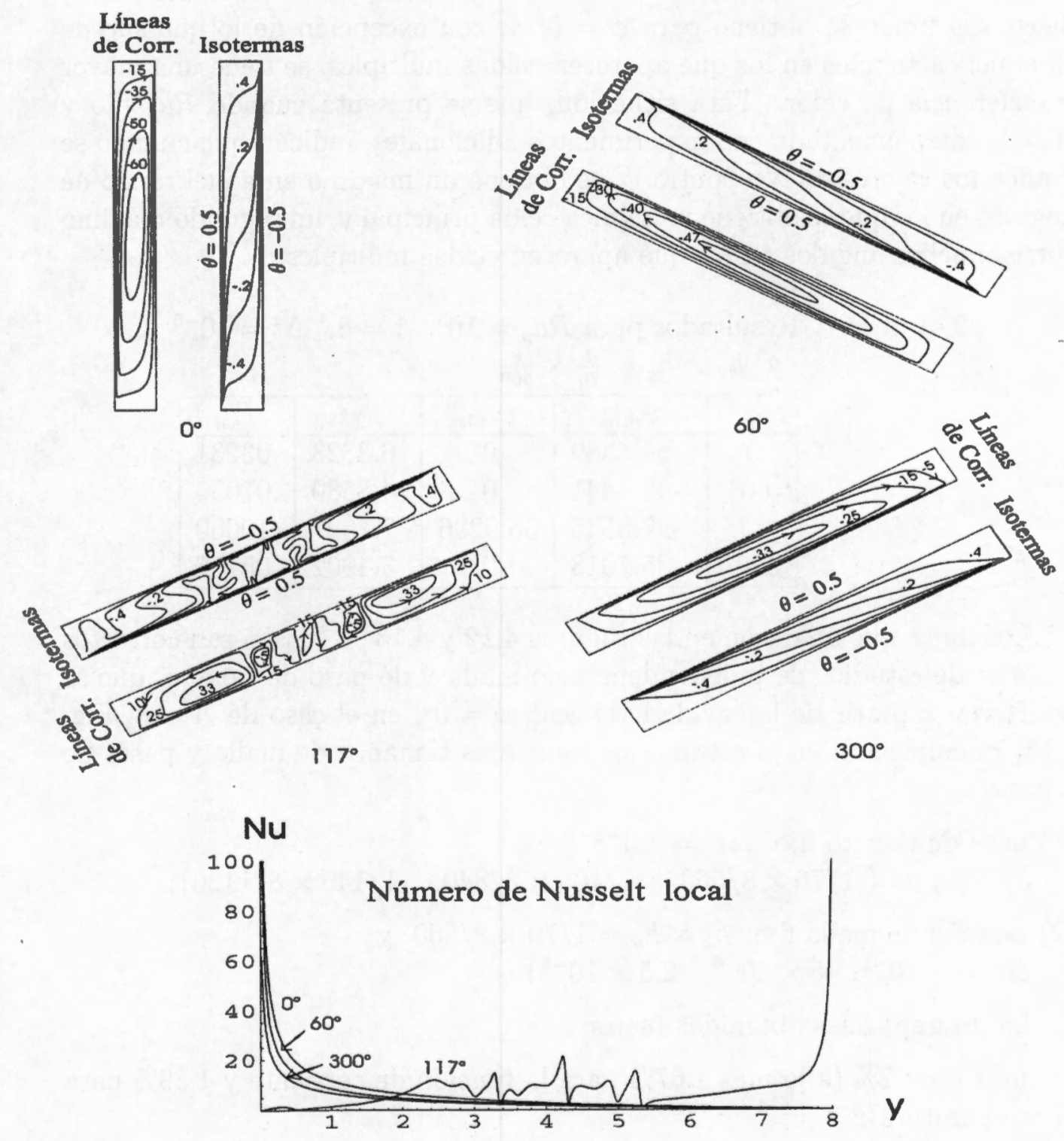

Fig. 4.13: $R a_{p}=10^{3}, A=8, h_{x} \times h_{y}=\frac{1}{70} \times \frac{8}{560}$ y $\Delta t=10^{-5}$. 
compleja. De manera similar a lo sucedido para $A=4$, no hay evidencia de la presencia de celdas secundarias, al menos para los ángulos considerados. La correspondiente Tabla P.7 confirma una vez más que el movimiento más fuerte del fluido se obtiene para $\phi=0^{\circ} \mathrm{y}$, con excepción de lo que sucede en aquellos ángulos en los que aparecen celdas múltiples, se tiene una mayor transferencia de calor. Esta situación, que se presenta cuando $R a_{p}$ fijo y $A>1$, complementada con experimentos adicionales, indican que cuando se grafica los valores de $\overline{N u}$ contra $\phi$, se obtiene un máximo en aquel rango de ángulos en los que se obtiene una única celda principal y, un segundo máximo entre aquellos ángulos en los que aparecen celdas múltiples.

Tabla P.7. Resultados para $R a_{p}=10^{3}, A=8, \Delta t=10^{-5}$

$\mathrm{y} h_{x} \times h_{y}=\frac{1}{70} \times \frac{8}{560}$.
\begin{tabular}{|c|c|c|c|c|}
\hline$\phi$ & $\psi_{\min }$ & $\psi_{\text {máx }}$ & $\overline{N u}$ & $T_{\text {est }}$ \\
\hline $0^{\circ}$ & -64.8389 & 0 & 6.3323 & .03231 \\
$60^{\circ}$ & -49.1441 & 0 & 4.8580 & .07036 \\
$117^{\circ}$ & -27.8545 & 36.9296 & 7.7607 & .10000 \\
$300^{\circ}$ & -35.7013 & 0 & 3.4902 & .05757 \\
\hline
\end{tabular}

Los flujos que aparecen en las Figuras 4.12 y 4.13 se consideran correctos a partir de estudios de independencia de malla y de paso de tiempo, que se realizaron a partir de la cavidad vertical, $\phi=0^{\circ}$; en el caso de $A=8$, Fig. 4.13 , se emplearon en el estudio los siguientes tamaños de malla y pasos de tiempo

(1) paso de tiempo fijo: $\Delta t=10^{-5} \mathrm{y}$

$h_{x} \times h_{y}=\{1 / 70 \times 8 / 560,1 / 105 \times 8 / 840,1 / 140 \times 8 / 1120\} ;$

(2) tamaño de malla fijo: $h_{x} \times h_{y}=1 / 70 \times 8 / 560$ y

$\Delta t=\left\{10^{-5}, 5 \times 10^{-6}, 2.5 \times 10^{-6}\right\}$.

La discrepancias obtenidas fueron :

(1) menos de $2 \%$ (a lo más $1.67 \%$ para la función de corriente y $1.59 \%$ para la temperatura);

(2) a lo más $5.16 \%$ (5.16\% para la función de corriente y $2.15 \%$ para la temperatura).

El valor mínimo de la función de corriente $\psi$ (el máximo siempre es cero) para cada caso es: 
(1) $\min =-64.8389,-64.8197,-64.8379$;

(2) mín $=-64.8389,-64.8415,-64.8351$.

De esta manera y en base a las pequeñas discrepancias observadas, así como a que no se evidenciaron cambios al considerar mallas más finas, los resultados que se muestran en la Figura 4.13 se consideran correctos. Para el caso más sencillo, cuando $A=4$, Fig. 4.12, sucede algo similar.

\section{3) Cavidad rectangular horizontal}

Concluimos esta sección presentado algunos resultados considerando ahora la cavidad rectangular horizontal, $A \leq 1$, en donde uno de los objetivos es realizar comparaciones con lo obtenido por Moya et al. [31] quienes resuelven el problema estacionario correspondiente a través de un método de falso transitorio para una cavidad horizontal que se calienta por debajo; por esta razón, como se señaló al inicio de esta sección de resultados, consideramos una condición de frontera para la temperatura ligeramente distinta a la de la subsección anterior; además, el problema es re-escalado con el objeto de que $0 \leq x, y \leq 1$, tal como se considera en [31]. De acuerdo a esta condición, el calentamiento y enfriamiento del fluido ocurre ahora en las paredes inferior y superior respectivamente, cuya diferencia de temperaturas, al igual que en la otra condición, sigue siendo de una unidad, mientras que las paredes laterales son adiabáticas. En los experimentos que presentamos, se consideran diversos valores de $R a_{p}$, de $A$ y de el ángulo de inclinación $\phi$.

Tabla P.8. Resultados para $R a_{p}=10^{2}$ y $\Delta t=2 \times 10^{-3}$.

\begin{tabular}{|c|c|c|c|c|c|l|}
\hline $\mathrm{A}$ & $h_{x} \times h_{y}$ & $\phi$ & $\psi_{\text {máx }}$ & $\overline{N u}$ & $\begin{array}{c}\text { Número } \\
\text { de celdas }\end{array}$ & $T_{\text {est }}$ \\
\hline$\frac{1}{3}$ & $\frac{1}{60} \times \frac{1}{40}$ & $10^{\circ}$ & 2.0409 & 2.8663 & 3 & 0.212 \\
& & $25^{\circ}$ & 2.3956 & 2.2494 & $1+$ sec. & 0.168 \\
& & $40^{\circ}$ & 2.8887 & 2.5491 & 1 & 0.338 \\
\hline$\frac{1}{10}$ & $\frac{1}{100} \times \frac{1}{20}$ & $10^{\circ}$ & 0.5860 & 2.7302 & 13 & 0.724 \\
& & $40^{\circ}$ & 0.8480 & 1.5218 & $1+$ sec. & 0.472 \\
& & $55^{\circ}$ & 1.0237 & 1.6240 & 1 & 0.452 \\
\hline
\end{tabular}

Algunos resultados obtenidos cuando $R a_{p}=10^{2}$ y dos valores distintos de $A$ se reportan en la Tabla P.8. Como se observa en ambos casos, el valor máximo de la función de corriente, $\psi_{\text {máx }}$, se incrementa con el ángulo, reafirmando lo que se obtuvo en la subsección anterior, es decir, el movimiento de 
fluido es mayor cuando se tiene una única celda convectiva; por su parte, el número global de Nusselt $\overline{N u}$ pareciera oscilar, sin embargo, como se comentó anteriormente $\mathrm{y}$ en base a experimentos realizados de manera complementaria, este efecto se presenta cuando $A<1$ y es provocado por la presencia de celdas convectivas múltiples; en estos casos, $\overline{N u}$ presenta un máximo entre aquellos ángulos donde aparece una celda principal y otro entre los ángulos donde surgen celdas múltiples. Moya et al. [31] corroboran esta situación, y presentan resultados para $R a_{p} \leq 10^{2}$ y tres distintas razones geométricas.

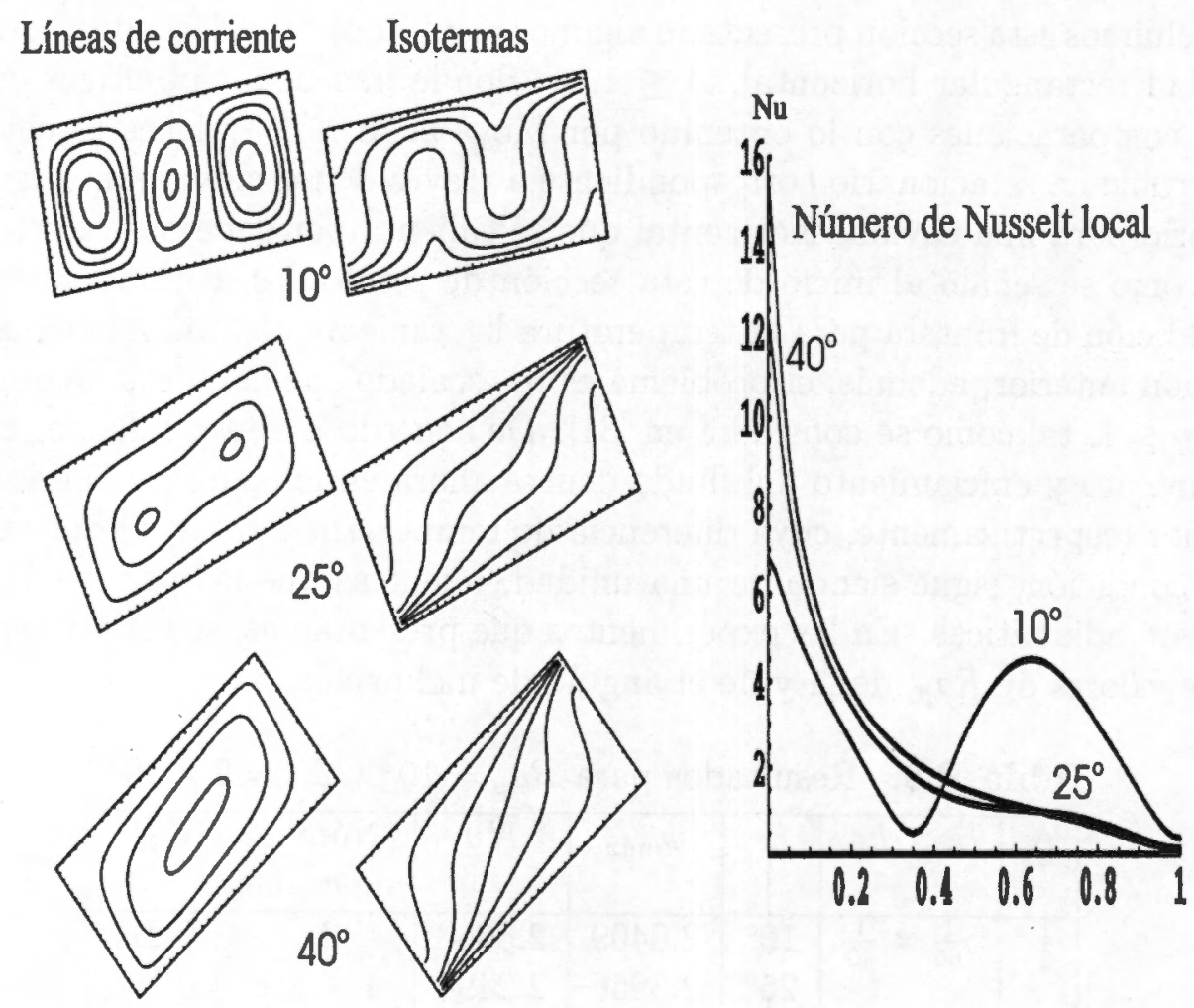

Fig. 4.14: $R a_{p}=10^{2}, A=\frac{1}{3}, h_{x} \times h_{y}=\frac{1}{60} \times \frac{1}{40}$ y $\Delta t=2 \times 10^{-3}$.

La Figura 4.14 muestra los resultados obtenidos para $R a_{p}=10^{2}, A=\frac{1}{3}$, y tres distintos ángulos, a partir de estos se observa que ahora el fluido se eleva sobre la pared derecha y desciende por la izquierda, lo que genera un movimiento en sentido contrario al reloj, esto es en dirección positiva, cuando aparece una única celda. Para el ángulo $\phi=10^{\circ}$ se observa la presencia de tres celdas primarias, la celda central girando en dirección negativa y las dos 
de los extremos en dirección contraria; por otro lado, las isotermas muestran dos regiones, cerca de la frontera caliente, donde el fluido proveniente de la pared superior, y por lo tanto más frío, desciende hacia el fondo de la cavidad. Estas regiones se caracterizan porque las isotermas se concentran en mayor número sobre la frontera inferior, lo que redunda en una mayor transferencia de calor, como lo indica la gráfica del número de Nusselt local. Los mínimos corresponden a aquellas regiones donde el fluido caliente del fondo asciende. Celdas secundarias hacen su aparición en $\phi=25^{\circ}$, y como se indicó anteriormente su presencia tiende a elevar, aunque de manera imperceptible, la transferencia de calor. Cuando $\phi=40^{\circ}$ se obtiene una celda principal y en este caso, la gráfica del número de Nusselt local muestra un único máximo, al inicio de la frontera inferior, y un único mínimo, al final; además al comparar esta gráfica con las de los otros ángulos, se observa que la mayor transferencia de calor, al menos hasta cerca de la mitad de la pared caliente, se alcanza cuando sólo aparece una celda convectiva.

Una situación similar se presenta cuando $A=\frac{1}{10}$, con la diferencia que para ángulos pequeños se llegan a obtener hasta trece celdas convectivas lo que implica que la gráfica del número de Nusselt local tiene siete máximos y el mismo número de mínimos. A pesar de lo anterior, y como lo muestra la Tabla P.8, el número de Nusselt global $\overline{N u}$ tiende a disminuir cuando la razón geométrica decrece, independientemente del ángulo considerado.

Tabla P.9. Angulo de transición para $R a_{p}=10^{2}$ y diversas razones geométricas.

\begin{tabular}{|c|c|}
\hline $\mathrm{A}$ & $\phi_{t}$ (transición) \\
\hline $1 / 2$ & $11^{\circ}$ \\
$1 / 3$ & $24^{\circ}$ \\
$1 / 4$ & $29^{\circ}$ \\
$1 / 8$ & $32^{\circ}$ \\
$1 / 10$ & $30^{\circ}$ \\
\hline
\end{tabular}

Los resultados obtenidos para $A=\frac{1}{3}$ concuerdan por completo con los que reportan Moya et al. [31], sin embargo y aunque los resultados correspondientes a $A=\frac{1}{10}$ muestran buena concordancia, existen algunas diferencias en cuanto al número de celdas convectivas que se obtienen cuando $\phi=10^{\circ}$, ya que ellos reportan sólo nueve celdas y por lo mismo siete máximos, y siete mínimos, en la gráfica del número de Nusselt local, mientras que en este trabajo se emplearon mallas de diferentes tamaños, y diversos pasos de 
tiempo, y los resultados para este ángulo mostraron que, dependiendo del tamaño de la malla, se obtiene una cantidad distinta de celdas: desde nueve celdas cuando se usa una malla uniforme de $\frac{1}{30} \times \frac{1}{30}$, hasta quince con una malla más fina de $\frac{1}{400} \times \frac{1}{40}$.

Por otra parte y como se desprende de los resultados obtenidos, la cantidad de celdas convectivas que aparecen en las líneas de corriente, cuando $A<1$, disminuye al aumentar el ángulo de la cavidad, hasta que sólo aparece una celda principal. Al ángulo en el que se pasa de tener celdas múltiples a una sola celda, se le llama ángulo de transición y aquí lo denotamos por $\phi_{t}$. De esta manera y con el objeto de determinar el ángulo de transición para $R a_{p}=10^{2}$ fijo y diferentes razones geométricas, se realizaron estudios para diversos tamaños de malla y pasos de tiempo, los resultados aparecen en la Tabla P.9. Podemos apreciar que el ángulo de transición $\phi_{t}$ para $A=\frac{1}{4}$, $A=\frac{1}{8}$ y $A=\frac{1}{10}$ difiere poco entre ellos, sin embargo esta diferencia es mayor para $A=\frac{1}{3}$ y mucho más notoria para $A=\frac{1}{2}$; en estas últimas dos razones geométricas, los ángulos de transición muestran una diferencia de $1^{\circ}$ respecto a los que obtuvieron Caltagirone y Bories [28] por una parte, y Moya et al. [31] por otra, sin embargo para los otros aspectos, los ángulos coinciden con los reportados por dichos autores.

Tabla P.10. Número de Nusselt global para $R a_{p}=10^{2}$ y $\Delta t=2 \times 10^{-3}$.

\begin{tabular}{|c|c|c|c|c|c|c|}
\hline A & $\phi$ & $\begin{array}{c}\overline{N u} \\
(\mathrm{C})\end{array}$ & $\begin{array}{c}\text { Número } \\
\text { de celdas }(\mathrm{C})\end{array}$ & $\begin{array}{c}\overline{N u} \\
(\mathrm{~B})\end{array}$ & $\begin{array}{c}\text { Número } \\
\text { de celdas (B) }\end{array}$ & $\begin{array}{c}\text { Diferencia } \\
\text { en } \overline{N u}(\%)\end{array}$ \\
\hline $1 / 2$ & $0^{\circ}$ & 2.65 & 3 & 2.70 & 3 & 2 \\
& $10^{\circ}$ & 2.60 & 3 & 2.38 & $1+$ sec. & 9 \\
& $15^{\circ}$ & 2.44 & 1 & 2.57 & 1 & 5 \\
\hline $1 / 4$ & $0^{\circ}$ & 2.67 & 5 & 2.75 & 5 & 3 \\
& $30^{\circ}$ & 2.57 & 1 & 2.37 & $1+$ sec. & 8 \\
& $40^{\circ}$ & 2.02 & 1 & 2.20 & 1 & 9 \\
\hline $1 / 8$ & $0^{\circ}$ & 2.64 & 11 & 2.70 & 11 & 2 \\
& $30^{\circ}$ & 2.45 & 5 & 2.25 & $1+$ sec. & 9 \\
& $40^{\circ}$ & 1.52 & - & 1.65 & 1 & 8 \\
\hline
\end{tabular}

La Tabla P.10 muestra una comparación de los valores de la transferencia de calor, a través del número de Nusselt global $\overline{N u}$, y del número de celdas para varias razones geométricas, cuando $R a_{p}=10^{2}$, obtenidos en este trabajo, indicados por (B), y los presentados por Caltagirone y Bories [28], indicados por $(\mathrm{C})$. Como puede notarse, la diferencia entre los valores respec- 
tivos de $\overline{N u}$ es menor que $9 \%$ en todos los casos, pero el número de celdas es distinto en algunos de éstos.

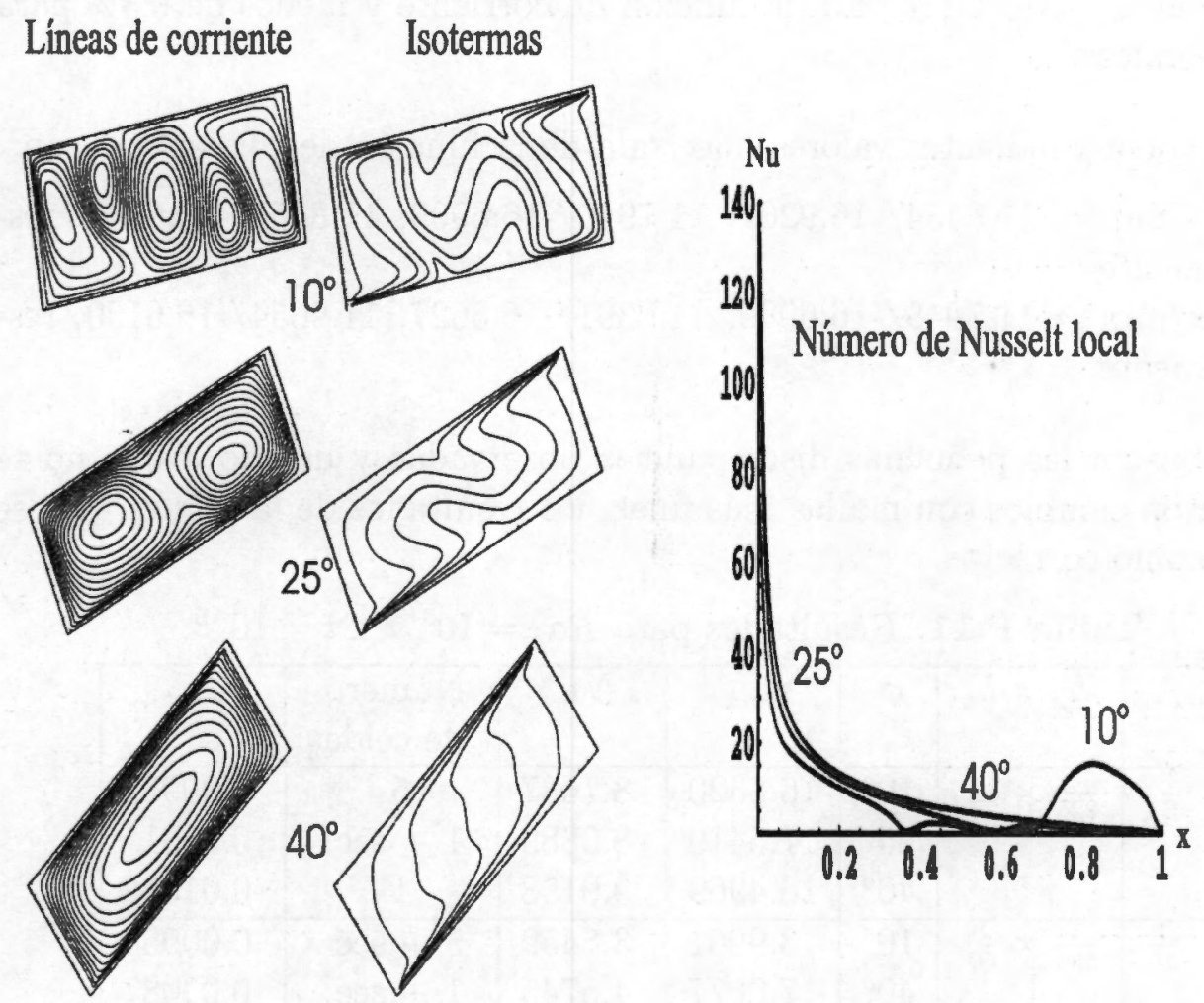

Fig. 4.15: $R a_{p}=10^{3}, A=\frac{1}{3}, h_{x} \times h_{y}=\frac{1}{225} \times \frac{1}{75}$ y $\Delta t=10^{-5}$.

Por otra parte, se realizaron experimentos adicionales para un valor más grande del número de Rayleigh, $R a_{p}=10^{3}$, y razones geométricas $A=\frac{1}{3}$ y $\frac{1}{10}$, los cuales no fueron considerados por Moya et al. [31], y hasta donde sabemos aquí se reportan por primera vez. Los resultados obtenidos para $A=\frac{1}{3}$ se validaron para el ángulo $\phi=0^{\circ}$, empleando tres tamaños de malla y tres pasos de tiempo distintos:

(1) paso de tiempo fijo: $\Delta t=10^{-5} \mathrm{y}$ $h_{x} \times h_{y}=\{1 / 150 \times 1 / 50,1 / 225 \times 1 / 75,1 / 300 \times 1 / 100\} ;$

(2) tamaño de malla fija: $h_{x} \times h_{y}=1 / 225 \times 1 / 75$ y $\Delta t=\left\{10^{-5}, \quad 5 \times 10^{-6}, \quad 2.5 \times 10^{-6}\right\}$.

Las discrepancias que se obtienen en cada caso son: 
(1) a lo más $4.4 \%$ (4.4\% para la función de corriente y $1.5 \%$ para la temperatura);

(2) a lo más $1.1 \%$ ( $1.1 \%$ para la función de corriente y menos de $0.3 \%$ para la temperatura).

Los correspondientes valores máx/mín de la función de corriente $\psi$ son:

(1) máx/mín = 11.6464/-16.9201, 11.7949/-16.6909, 11.8455/-16.6041, respectivamente;

(2) máx $/$ mín $=11.7949 /-16.6909,11.8391 /-16.6627,11.9054 /-16.6190$, respectivamente.

En base a las pequeñas discrepancias observadas y debido a que no se observaron cambios con mallas más finas, los resultados de la Figura 4.15 se toman como correctos.

Tabla P.11. Resultados para $R a_{p}=10^{3}$ y $\Delta t=10^{-5}$.

\begin{tabular}{|c|c|c|c|c|c|c|}
\hline $\mathrm{A}$ & $h_{x} \times h_{y}$ & $\phi$ & $\psi_{\max }$ & $\overline{N u}$ & $\begin{array}{l}\text { Número } \\
\text { de celdas }\end{array}$ & $T_{\text {est }}$ \\
\hline$\frac{1}{3}$ & $\frac{1}{225} \times \frac{1}{75}$ & $10^{\circ}$ & 16.8320 & 8.7687 & 5 & 0.00931 \\
& & $25^{\circ}$ & 17.5419 & 8.0889 & $1+$ sec. & 0.01011 \\
& & $40^{\circ}$ & 16.4969 & 9.9158 & 1 & 0.01312 \\
\hline$\frac{1}{10}$ & $\frac{1}{500} \times \frac{1}{50}$ & $10^{\circ}$ & 3.9961 & 3.6459 & $5+$ sec & 0.00956 \\
& & $40^{\circ}$ & 7.0677 & 4.5745 & $1+$ sec. & 0.03087 \\
& & $55^{\circ}$ & 7.4627 & 5.2531 & 1 & 0.03007 \\
\hline
\end{tabular}

Los ángulos considerados para este caso, muestran un comportamiento similar al observado para $R a_{p}=10^{2}$, esto es, las líneas de corriente se ordenan en celdas múltiples para ángulos pequeños, como sucede para $\phi=10^{\circ}$ en donde se obtienen cinco celdas, sin embargo, estas celdas al igual que las isotermas muestran distorsiones; al aumentar el ángulo hasta $\phi=25^{\circ}$ se obtiene una celda principal que enmarca a dos grandes celdas secundarias, cada una de las cuales ocupa casi por completo la mitad de la cavidad; y para ángulos mayores, como $\phi=40^{\circ}$, las celdas secundarias desaparecen y se observa una única celda principal. La respectiva gráfica del número de Nusselt local muestra que existe más transferencia de calor que la obtenida para $R a_{p}=10^{2}$ para los mismos ángulos; además, como era de esperarse, la mayor transferencia de calor se presenta en el extremo izquierdo de la frontera caliente, pero cuando $\phi=10^{\circ}$, aparece otro máximo cerca del extremo 
derecho, lo que es consecuencia de la pequeña celda que se localiza hacia el final de la pared inferior, y que acarrea el fluido más frío proveniente de la pared superior hacia la superficie caliente del fondo, lo que a su vez genera un aumento en la transmisión de calor en esta zona, y por lo tanto de $\overline{N u}$.

En la Tabla P.11 se muestran algunos resultados para $A=\frac{1}{3}$ y $A=\frac{1}{10}$ con mallas adecuadas. Puede observarse que los valores de $\psi_{\operatorname{máx}}$ y $\overline{N u}$ muestran por una parte, un claro incremento respecto a los obtenidos para $R a_{p}=10^{2}$, Tabla P.8, esto indica, como ya se ha mencionado, un movimiento más fuerte del fluido, y consecuentemente una mayor transferencia de calor; y por otro lado, se advierte que estos valores disminuyen si la razón geométrica disminuye, manteniendo $R a_{p}$ fijo, y ambos muestran nuevamente ser función del ángulo $\phi$. Además, el ángulo de transición es $\phi_{t}=24^{\circ}$ para $A=\frac{1}{3} \mathrm{y}$ $\phi_{t}=27^{\circ}$ para $A=\frac{1}{10}$, cada uno de los cuales es igual, en el primer caso, y menor, en el segundo, que los correspondientes obtenidos para $R a_{p}=10^{2}$. 


\section{Capítulo 5}

\section{Conclusiones}

En este trabajo hemos presentado esquemas numéricos para tratar el flujo de convección natural en fluidos térmicos, viscosos e incompresibles, en una región cerrada y acotada, en medios libres y en medios porosos, gobernados matemáticamente por la aproximación de Boussinesq no-estacionaria. Para cada medio se consideraron dos formulaciones distintas, una en variables primitivas y otra en variables secundarias; en cada una de estas formulaciones, los esquemas numéricos tienen la propiedad de tener una estructura común para cada medio.

La eficiencia de los procedimientos numéricos se manifiesta al aplicarlos al caso de flujos térmicos e isotérmicos en cavidades rectangulares, con valores moderados y grandes de $R a\left(R a_{l} \circ R a_{p}\right)$ o de $R e$, para varias razones geométricas $A$ y para diversos ángulos $\phi$. En los resultados obtenidos para flujos de convección natural, en ambos medios se pudo observar algunas características comunes, o dispares, que a continuación se describen: 1) las mallas empleadas en medios libres son considerablemente gruesas, mientras que en medios porosos esta situación es más severa ya que al aumentar $R a_{p}$ se requiere de un mayor refinamiento en la malla y en el paso de tiempo, aún para flujos en la cavidad unitaria. 2) En medios libres se obtienen flujos de estado estacionario para $A \leq 3$, y $R a_{l} \leq 10^{7}$, y flujos dependientes del tiempo para $A=8$ con $R a_{l}=3.4 \times 10^{5}$ y $10^{6}$; en medios porosos, se obtienen flujos estacionarios en la cavidad unitaria para $R a_{p} \leq 10^{4}$, y en la cavidad rectangular para $A \leq 10$ con $R a_{p}=10^{2}$ y $10^{3}$. 3) En ambos medios, un aumento en la altura de la cavidad, sin inclinación y con $\mathrm{Ra}$ fijo, incrementa el movimiento del fluido y el tiempo para alcanzar el estado estacionario, pero disminuye la transferencia de calor global; sin embargo, en flujos depen- 
dientes del tiempo, la transferencia de calor global muestra ligeros cambios en el tiempo, mientras que la local muestra fluctuaciones, las cuales son mayores, en número y en magnitud, para $R a_{l}=10^{6}$, en comparación con las se obtienen para $R a_{l}=3.4 \times 10^{5}$. 4) En ambos medios, un aumento en el valor de $R a=R a_{l} \circ R a_{p}$, en una cavidad con altura fija y sin inclinación, conduce a un movimiento más activo del fluido, a mayor transferencia de calor global y, en el caso de flujos que llegan el estado estacionario, a un tiempo menor para alcanzarlo. 5) Un aumento en el ángulo de inclinación de la cavidad implica, en medios libres, un descenso en la transferencia de calor, tanto en la cavidad cuadrada con Ra fijo, como en la cavidad con $A=8$ y $R a_{l}=3.4 \times 10^{5}$ al tiempo $T=50$; aunque en este último caso la transferencia de calor disminuye ligeramente para los ángulos considerados. En medios porosos, el movimiento del fluido aumenta y la transferencia de calor global oscila; además, para ángulos cercanos a $\phi=90^{\circ}$ el flujo se desarrolla en celdas contiguas que rotan en direcciones opuestas, alternadas, lo que ocasiona que la transferencia de calor local presente varios máximos; la cantidad de máximos es mayor cuanto más alta sea la cavidad, esto es, a mayor $A$ se tienen mayor cantidad de celdas en el patrón del flujo y, por lo tanto, más zonas donde la transferencia de calor aumenta.

Resumiendo para flujos estacionarios, en cualquiera de los dos medios, un aumento conjunto en el valor de $A$ y de $R a$, en la cavidad sin inclinación, por lo general conduce a un aumento, más notorio, en la actividad del fluido, aunque no necesariamente en la transferencia de calor, que cuando sólo cambia uno de ellos; si, además, $\phi \neq 0^{\circ}$, el flujo puede ser bastante complejo en algunos ángulos.

A partir de los resultados obtenidos, consideramos que los esquemas numéricos propuestos se pueden aplicar para tratar el problema de convección mixta en dos dimensiones, y por otro lado, se pueden extender y/o adaptar para tratar problemas más complejos como el fenómeno de bifurcación y de inestabilidad relacionado con valores grandes del número de Rayleigh y de la razón geométrica; flujos multifásicos, en donde la descripción de los fenómenos y de los movimientos interfaciales requiere de esquemas numéricos que proporcionen alta resolución y exactitud; el problema de convección natural o mixta en tres dimensiones en donde por las características del problema, es conveniente emplear los esquemas respectivos en variables primitivas o adaptar los correspondientes en variables secundarias, en términos del vector potencial, en lugar de la función de corriente, y el correspondiente vector vorticidad. 


\section{Bibliografía}

[1] White F. M., Mecánica de Fluidos. Ed. McGraw-Hill México, 1988.

[2] Phillips O. M.,Flow and Reactions in Permeable Rocks. Cambridge University Press, 1991.

[3] Lin C. C., Segel L. A., Mathematics Applied to Deterministic Problems in the Natural Sciences. Macmillan Publishing Co., Inc., New York, 1974.

[4] Landau L. D. and Lifschitz E. M., Fluid Mechanics, second edition, Pergamon Press, Inc., New York, 1989.

[5] Bear, Jacob, Dynamics of Fluids in Porous Media, American Elsevier Publishing Company, Inc., New York, 1972.

[6] Lions J. L., Temam R., Wang S. Models for the Coupled Atmosphere and Ocean. Computational Mechanics Advances I, 1 (1) (1993), pp. 1-54.

[7] Lions J. L., Temam R., Wang S. Mathematical Theory for Coupled Atmosphere and Ocean Models, J. M. P. A., 1994.

[8] Temam R. Navier-Stokes Equations, Theory and Numerical Analysis. AMS CHELSEA PUBLISHING; American Mathematical Society, Providence, Rhode Island, USA, 2001.

[9] Chen Z., Ewing R. E. Mathematical Analysis for Reservoir Models. SIAM Journal of Mathematical Analysis, 30 (1999), pp. 431-453.

[10] Pironneau O., Méthodes des Éléments Finis pour les Fluids. MASSON, Paris, 1988. 
[11] Goyon O., High Reynolds number solutions of Navier-Stokes equations using incremental unknowns, Comp. Methods Appl. Mech. Engrg., 130 (1996), pp. 319-335.

[12] Nicolás A., Bermúdez B., $2 D$ thermal/isothermal incompressible viscous flows. International Journal for Numerical Methods in Fluids, 48 (2005), pp. 349-366.

[13] Henkes R. A. W. M. and Hoogendoorn C. J., Scaling of laminar naturalconvection flow in a heated square cavity. Int. J. Heat and Mass Transfer 36 (11) (1993), pp. 2913-2925.

[14] Hamady F. J., Lloyd J. R., Yang H. Q. and Yang K. T., Study of local natural convection heat transfer in an inclined enclosure. Int. J. Heat and Mass Transfer 32 (9) (1989), pp. 1697-1708.

[15] Bermúdez B. and Nicolás A., An Operator splitting numerical scheme for thermal/isothermal incompressible viscous flows. Int. J. Numer. Methods Fluids 29 (1999), pp. 397-410.

[16] Ding H., Shu C., Yeo K. S., Xu D., Development of least-square-based two-dimensional finite-difference schemes and their application to simulate natural convection in a cavity. Computers \& Fluids, 33 (2004), pp. 137-154.

[17] Christon M. A.,Gresho P. M. and Sutton S. B., Computational predictability of time-dependent natural convection flows in enclosures (including a benchmark solution, Int. J. Numer. Methods Fluids, 40 (2002), pp. 953-980.

[18] Xin S. and Le Quéré P., An extended Chebyshev pseudo-spectral benchmark for the 8:1 differentially heated cavity. Int. J. for Num. Meth. in Fluids 40 (2002), pp. 981-998.

[19] Auteri F. and Parolini N., Numerical investigation of the first instabilities in the differentially heated 8:1 cavity. Int. J. for Num. Meth. in Fluids 40 (2002), pp. 1121-1132.

[20] Glowinski R., Handbook of Numerical Analysis: Numerical Methods for Fluids (Part 3), North-Holland, 2003. 
[21] Karniadakis G. E., Israeli M. and Orszag S. A. High-order splitting methods for the incompressible Navier-Stokes equations, J. of Comp. Physics, 97 (1991), pp. 414-443.

[22] Orszag S., Israeli M., Deville M. Boundary conditions for incompressible flows. Journal of Scientific Computing, 1 1986, pp.75-111.

[23] Gresho P. and Sani R. On pressure boundary conditions for incompressible Navier-Stokes equations. International Journal for Numerical Methods in Fluids, 7 1987, pp. 1111-1145.

[24] Weinan E. and Liu J. G. Projection Method I: Convergence and numerical boundary layers, SIAM J. Numer. Anal., 32 (4) (1995), pp. 1017-1057.

[25] Pan T. W. and Glowinski R. A Projection/Wave-Like Equation Method for the Numerical Simulation of Incompressible Viscous Fluid Flow Modeled by the Navier-Stokes Equations. Computational Fluid Dynamics Journal, 9 (2) (2000), pp. 28-42.

[26] Sahin M. and Owens R. G. A novel fully implicit finite volume method applied to the lid-driven cavity problem -part I: high Reynolds number flow calculations. International Journal for Numerical Methods in Fluids, 42, 2003, pp. 57-77.

[27] Holst P. H. and Aziz K., Transient three-dimensional natural convection in confined porous media. Int. J. Heat and Mass Transfer 15 (1972), pp. 73-90.

[28] Caltagirone J. P. and Bories S., Solutions and stability criteria of natural convective flow in an inclined porous layer, J. Fluid Mech., 155 (1985), pp. 267-287.

[29] Baytas A. C., Entropy generation for natural convection in a inclined porous cavity. Int. J. Heat and Mass Transfer 43 (2000), pp. 2089-2099.

[30] Saeid N. H. and Pop I., Transient free convection in a square cavity filled with porous medium. Int. J. of Heat and Mass Transfer 47 (2004), pp. 1917-1924. 
[31] Moya S. L., Ramos E., Sen M., Numerical study of natural convection in a tilted rectangular porous material, Int. J. Heat and Mass Transfer 30 (4) (1987), pp. 741-756.

[32] Sen M., Vasseur P. and Robillard L., Multiple steady states for unicellular natural convection in an inclined porous layer. Int. J. Heat and Mass Transfer 30 (10) (1987), pp. 2097-2113.

[33] Foias C., Manley O., Rosa R., Temam R. Navier-Stokes Equations and Turbulence. Cambridge University Press, 2001.

[34] Mohammadi B. and Pironneau O., Analysis of the K-Epsilon TURBULENCE MODEL. John Wiley and Sons \& Masson, 1994.

[35] Báez E., Bermúdez B. y Nicolás A. Convección Natural en Medios Porosos y Libres: Simulación Numérica. Rev. Mex. de Física 50, (1) (2004), pp. 35-48.

[36] Báez E. and Nicolás A. 2D Natural Convection Flows in Tilted Cavities: Porous Media and Homogeneous Fluids. Int. J. of Heat and Mass Transfer 49 (25) (2006), pp. 4773-4785.

[37] Báez E. and Nicolás A. Natural Convection Fluid Flow and Heat Transfer in Porous Media. J. of Mech. of Material and Structures 2 (8) (2007), pp. 1571-1584.

[38] Griebel M., Dornseifer T. and Neunhoeffer T., Numerical Simulation in Fluid Dynamics, SIAM, Philadelphia, 1998.

[39] Tilton N. and Cortelezzi L., Linear stability analysis of pressure-driven flows in channel with porous walls, J. Fluid Mech., 604 (2008), pp. 411445 .

[40] Whitaker S., The Forchheimer Equation: A Theoretical Development, Transport in Pouous Media, 25 1996, pp. 27-61

[41] Gunzburger, M. D. Finite element methods for viscous incompressible flows: A guide to theory, practice and algorithms, Academic Press, New York, 1989.

[42] Bristeau M. O., Glowinski R., Periaux J. Numerical Methods for the N-S Equations, Computer Physics Report, 6 (1987), pp. 73-187. 
[43] Badalassi V. E., Ceniceros H. D., Banerjee S. Computation of multiphase systems with phase field models. Journal of Computational Physics, 190 (2003), pp. 371-397.

[44] Sani R. L., Shen J., Pironneau O., Gresho P. M. Pressure boundary condition for the time-dependent incompressible Navier-Stokes equations. International Journal for Numerical Methods in Fluids, 50 2006, pp. 673-682.

[45] Adams J., Swarztrauber P. and Sweet R., FISHPACK: A Package of Fortran Subprograms for the Solution of Separable Elliptic PDE's, The National Center for Atmospheric Research, Boulder, Colorado, 1980.

[46] Sweet R., A cyclic reduction algorithm for solving block tridiagonal systems of arbitrary dimensions, SIAM Journal on Numer. Anal. 14 (1977), pp. 706-720.

[47] Schreiber R., Keller H.B. Driven cavity fiow by efficient numerical techniques, Journal of Computational Physics 40 (1983), pp. 310-333.

[48] Ghia U., Ghia N., Shin C. T. High-Re solutions for incompressible flow using the Navier-Stokes equations and a multigrid method. Journal of Computational Physics 48 (1982), pp. 387-411.

[49] Erturk E., Corke, T. C., Gökçöl C. Numerical solutions of 2-D steady incompressible driven cavity flow at high Reynolds numbers. International Journal for Numerical Methods in Fluids 48 (2005), pp. 747-774.

[50] Sahin M., Owens R. G. A novel fully implicit Finite volume method applied to the lid-driven cavity problem.Part I: High Reynolds number flow calculations. International Journal Numerical Methods in Fluids 42 (2003), pp. 57-77.

[51] Glowinski R., Finite element methods for the numerical simulation of the numerical simulation of incompressible viscous flow. Introduction to the control of the Navier Stokes equations, Lectures in Applied Mathematics, AMS, Providence, RI, 28 (1991), pp. 219.

[52] Peyret R. and Taylor T. D., Computational Methods for Fluid Flow, Springer-Verlag, Berlín, 1983. 
[53] Dean E. J., Glowinski R., Pironneau O., Iterative solution of the stream function-vorticity formulation of the Stokes problem, applications to the numerical simulation of incompressible viscous flow, Comp. Methods Appl. Mech. Engrg., 87 (1991), pp. 117-155.

[54] Kuyper R. A., Van Der Meer Th. H., Hoogendoorn C. J. and Henkes R. A. W. M., Numerical study of laminar and turbulent natural convection in an inclined square cavity. Int. J. Heat and Mass Transfer 36 (11) (1993), pp. 2899-2911.

[55] Markatos N. C. and Pericleous K.A., Laminar and turbulent natural convection in an enclosed cavity, Int. J. Heat and Mass Transfer 27(5) (1984), pp. 755-772. 


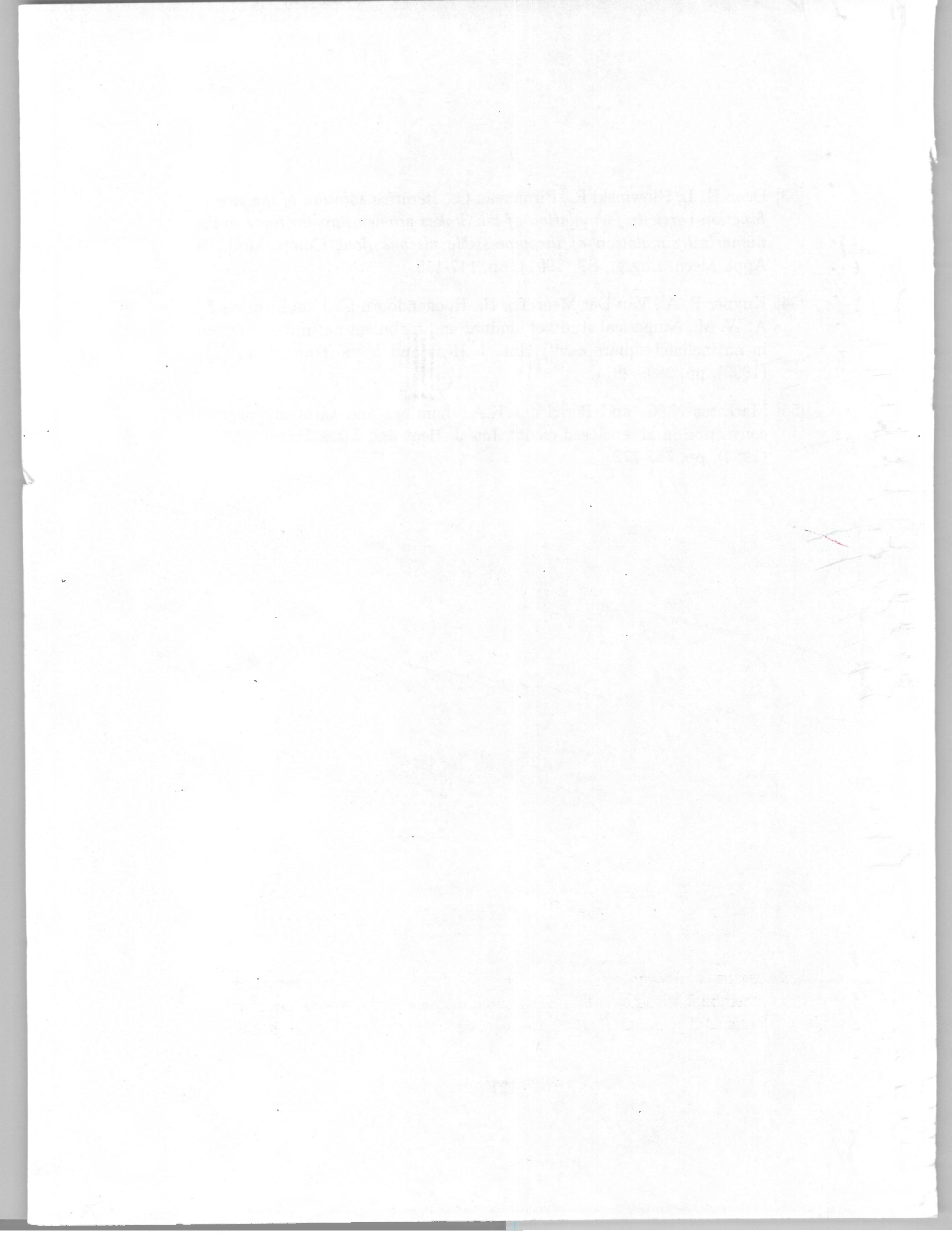

